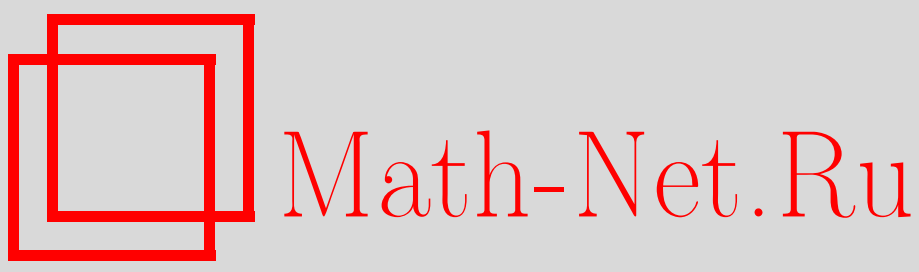

Л. В. Кузьмин, Некоторые замечания о $\ell$-адическом регуляторе. III, Изв. РАН. Сер. матем., 1999, том 63, выпуск 6, 29-82

DOI: https://doi.org/10.4213/im268

Использование Общероссийского математического портала Math-Net.Ru подразумевает, что вы прочитали и согласны с пользовательским соглашением

http://www . mathnet.ru/rus/agreement

Параметры загрузки:

IP : 54.162 .127 .20

26 апреля 2023 г., 13:12:32 
УДК 519.4

\section{Л.В. Кузьмин}

\section{Некоторые замечания о $\ell$-адическом регуляторе. III}

Пусть $K$ - конечное расширение поля рациональных $\ell$-адических чисел $\mathbb{Q}_{\ell}$ и $K_{\infty}-$ круговое $\mathbb{Z}_{\ell}$-расширение поля $K$. Для промежуточного поля $K_{n}$ расширения $K_{\infty} / K$ пусть $U\left(K_{n}\right)$ - группа единиц поля $K_{n}$ и $U\left(K_{n}\right)^{\perp}=\{x \in$ $K_{n} \mid \operatorname{Sp}_{K_{n} / \mathbb{Q}_{\ell}}(x \log u) \in \mathbb{Z}_{\ell}$ для всех $\left.u \in U\left(K_{n}\right)\right\}$, где $\log : U\left(K_{n}\right) \rightarrow K_{n}-$ $\ell$-адический логарифм. Дается приближенное описание группы $U\left(K_{n}\right)^{\perp}$. Доказательства основаны на использовании рядов Лорана с целыми коэффициентами и бесконечной главной частью.

Библиография: 9 наименований.

\section{Введение}

Пусть $K$ - поле алгебраических чисел, $\ell$ - фиксированное простое число и $U(K)$ - группа единищ поля $K$. Тогда существует диагональное вложение $i: U(K) \hookrightarrow \prod_{v \mid \ell} U\left(K_{v}\right)$, где $K_{v}$ - пополнение $K$ относительно точки $v, U\left(K_{v}\right)-$ группа единищ $K_{v}$ и $v$ пробегает все точки поля $K$, лежащие над $\ell$. Знаменитая гипотеза Леопольдта утверждает, что $i$ индуцирует вложение

$$
i_{1}: U(K)[\ell] \hookrightarrow \mathscr{A}(K):=\prod_{v \mid \ell} U\left(K_{v}\right),
$$

где $U(K)[\ell]$ - про- $\ell$-пополнение группы $U(K)$ и $U\left(K_{v}\right)$ - единицы поля $K_{v}$.

В [2] была высказана следующая гипотеза, являющаяся усилением гипотезы Леопольдта.

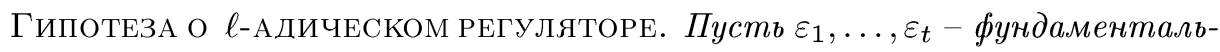
ная система единич поля $K$. Пусть $\log : \mathscr{A}(K) \rightarrow \prod_{v \mid \ell} K_{v}$ - отображение, совпадающее на каждой компоненте с $\ell$-адическим логарифмом. Тогда $\ell$-адический регулятор, определяемый формулой

$$
R_{\ell}(K)=\operatorname{det}\left(\operatorname{Sp}_{K / \mathbb{Q}}\left(\log \varepsilon_{i} \cdot \log \varepsilon_{j}\right)\right), \quad 1 \leqslant i, j \leqslant t
$$

отличен от нуля для любого поля $K$. Здесь $\mathrm{Sp}_{K / \mathbb{Q}}$ обозначает след из алгебрь $K \otimes_{\mathbb{Q}} \mathbb{Q}_{\ell}=\prod_{v \mid \ell} K_{v}$ в $\mathbb{Q}_{\ell}$.

Таким образом, наша гипотеза утверждает, что $i_{1}$ - вложение и, кроме того, скалярное произведение

$$
\mathscr{A}(K) \times \mathscr{A}(K) \rightarrow \mathbb{Q}_{\ell}, \quad(x, y) \rightarrow \operatorname{Sp}_{K / \mathbb{Q}_{\ell}}(\log x \cdot \log y), \quad x, y \in \mathscr{A}(K),
$$

остается невырожденным при ограничении на $\operatorname{Im} i_{1}$.

Работа выполнена при поддержке РФФИ (проект № 97-01-00516).

$$
\text { (C) Л.в. Кузьмин } 1999
$$


Наряду с $R_{\ell}(K)$ удобно определить относительный $\ell$-адический регулятор. Если задано конечное расширение полей алгебраических чисел $K / k$ и $\varepsilon_{1}, \ldots, \varepsilon_{r}-$ некоторый базис группы относительных единиц $U(K / k)$, то относительный $\ell$-адический регулятор определяется формулой

$$
R_{\ell}(K / k)=\operatorname{det}\left(\operatorname{Sp}_{K / \mathbb{Q}_{\ell}}\left(\log \varepsilon_{i} \cdot \log \varepsilon_{j}\right)\right), \quad 1 \leqslant i, j \leqslant r .
$$

СЛАБАЯ ГИПОТЕЗА О $\ell$-АДИЧЕСКОМ РЕГУЛЯТОРЕ. Пусть $K$ - nоле алгебрачческих чисел и $K_{\infty}$ - круговое $\mathbb{Z}_{\ell}$-расширение поля $K$. Тогда существует индекс $n_{0}$, зависящий только от расширения $K_{\infty} / K$ и такой, что $R_{\ell}\left(K_{n} / K_{n_{0}}\right) \neq 0$ для всех $n>n_{0}$.

В [2], [3] было дано доказательство этих гипотез для некоторых частных случаев.

Очевидно, что последняя гипотеза предполагает одновременное рассмотрение произведений (0.3) для всех промежуточных полей $K_{n}$ расширения $K_{\infty} / K$. Удобная для этого конструкция была предложена в [3], хотя некоторые идеи, относяшиеся к этой конструкции, восходят еще к работе Ивасавы [1].

Пусть $K$ - конечное круговое расширение $\mathbb{Q}_{\ell}$ и $K_{\infty}=\bigcup K_{n}-$ круговое $\mathbb{Z}_{\ell}$-расширение поля $K$. Пусть $U\left(K_{\infty}\right)=\lim _{\longleftarrow} U\left(K_{n}\right) / \mu\left(K_{n}\right)$, где $\mu\left(K_{n}\right)$ - группа всех корней из единицы в $K_{n}$. В [3] было дано определение модуля Галуа $V\left(K_{\infty}\right)$ такого, что $U\left(K_{\infty}\right) \subset V\left(K_{\infty}\right)$ и $V\left(K_{\infty}\right) / U\left(K_{\infty}\right) \cong \lim \mu\left(K_{n}\right)$ (не канонически). Кроме того, было доказано существование на $V\left(K_{\infty}\right)$ невырожденного внутреннего произведения

$$
V\left(K_{\infty}\right) \times V\left(K_{\infty}\right) \longrightarrow \Lambda \cong \mathbb{Z}_{\ell}[[X]]
$$

т.е. произведения, индуцируюшего изоморфизм $V\left(K_{\infty}\right) \cong \operatorname{Hom}_{\Lambda}\left(V\left(K_{\infty}\right), \Lambda\right)$, где $\Lambda=\mathbb{Z}_{\ell}[[\Gamma]]-$ пополненная групповая алгебра группы Галуа $\Gamma=G\left(K_{\infty} / K\right)$.

В случае, когда $K$ - поле алгебраических чисел, имеюшее абелевы пополнения относительно всех точек $v \mid \ell,(4)$ приводит к полулокальному внутреннему произведению

$$
\prod_{v \mid \ell} V\left(K_{\infty, v}\right) \times \prod_{v \mid \ell} V\left(K_{\infty, v}\right) \longrightarrow \Lambda
$$

которое имеет много полезных приложений [4]-[6].

В [7] было получено новое более простое доказательство основного локального результата, позволяющего строить произведения (0.4) и (0.5). Именно, пусть $k_{n}=H\left(\zeta_{n}\right)$, где $H$ - конечное неразветвленное расширение $\mathbb{Q}_{\ell}$ и $\zeta_{n}-$ первообразный корень из единицы степени $\ell^{m}, m=n+1$ при $\ell \neq 2$ и $m=n+2$ при $\ell=2$. Пусть $V\left(k_{n}\right)$-мультипликативная группа, порожденная $U\left(k_{n}\right)$ и элементом $\sqrt[\ell^{m}]{u_{n}}$, где $u_{n} \in U\left(k_{n}\right)$ и $k_{n}\left(\sqrt[\ell^{m}]{u_{n}}\right) / k_{n}$ - неразветвленное расширение степени $\ell^{m}$. Положим

$$
\Phi_{n}=\ell^{-m-1}\left[\delta \operatorname{Sp}_{k_{n} / H}+\ell \operatorname{Sp}_{k_{n} / k_{1}}+\sum_{i=0}^{n-1} \mathscr{F}_{n}^{(i)}\right] \in \mathbb{Q}_{\ell}\left[G\left(k_{n} / H\right)\right],
$$

где $\mathscr{F}_{n}^{(i)}=\ell \operatorname{Sp}_{k_{n} / k_{n-i}}-\operatorname{Sp}_{k_{n} / k_{n-i-1}}$ и $\delta=-1$ при $\ell \neq 2, \delta=0$ при $\ell=2$. В [7] была доказана 
ТЕОРемА А. Для любого $n \geqslant 1$ справедливо равенство

$$
\operatorname{Ker} \operatorname{Sp}_{k_{n} / k_{0}}\left(V\left(k_{n}\right)^{\perp}\right)=(\gamma-1) \Phi_{n}\left(\log V\left(k_{n}\right)\right),
$$

әде $\gamma$ - некоторая образующая группь $G\left(k_{n} / k_{0}\right) u$

$$
V\left(k_{n}\right)^{\perp}=\left\{x \in k_{n} \mid \operatorname{Sp}_{k_{n} / \mathbb{Q}_{\ell}}(x \log y) \in \mathbb{Z}_{\ell} \quad \text { для всех } \quad y \in V\left(k_{n}\right)\right\} .
$$

Наиболее трудную часть этой теоремы составляет

Теорема В. В обозначениях теоремы А выполняется включение

$$
(\gamma-1) \Phi_{n}\left(\log U\left(k_{n}\right)\right) \subseteq U\left(k_{n}\right)^{\perp}
$$

В настоящей работе мы начинаем изучение рассмотренных вьше вопросов без предположения, что рассматриваемые локальные поля являются абелевыми.

Итак, пусть $K$ - произвольное конечное расширение поля $\mathbb{Q}_{\ell}$, поля́ $k_{n}$ определены, как выше, и $K_{n}=K \cdot k_{n}$. В этом случае снова определены модули Галуа $V\left(K_{n}\right)$ и $V\left(K_{\infty}\right)=\lim V\left(K_{n}\right)$, однако легко можно показать, что внутреннего произведения (4) в общем случае не существует. Что касается теоремы А, то заметим, что поля $K_{n}$ начинают регулярно вести себя только для достаточно больших $n$, поэтому правильная формулировка теоремы А для произвольного локального поля $K$ требует замены множителя $(\gamma-1)$ на $\left(\gamma_{p}-1\right)$, где $\gamma_{p}$ - образуюшая групшы $G\left(K_{n} / K_{p}\right)$ и индекс $p$ зависит только от расширения $K_{\infty} / K$. К сожалению, это обобщение неверно в общем случае, а также и теорема В перестает быть верной для неабелева локального поля $K$. Соответствуюшие контрпримеры будут рассмотрены в следующей работе из этой серии. Тем не менее, оказывается, что верен следующий ослабленный вариант теоремы В.

Основная теОРема. Пусть $K$ - произвольное конечное расширение $\mathbb{Q}_{\ell}$, $K_{n}=K \cdot k_{n}$ u $K_{\infty}=\bigcup K_{n}$. Тогда существуют индекс $p$ и неотрицательная константа $C$, зависящие только от расширения $K_{\infty} / K$ и такие, что

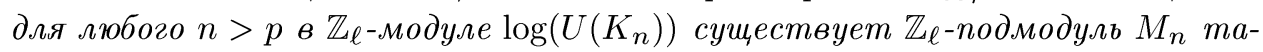
кой, что группа $\log \left(U\left(K_{n}\right)\right) / M_{n}$ может быть порождена не более чем $C n$ образующ, мми, $и$

$$
\left(\gamma_{p}-1\right) \Phi_{n}\left(M_{n}\right) \subseteq U\left(K_{n}\right)^{\perp} .
$$

При этом р и С могут быть определены әффективно.

Доказательство этой теоремы и составляет основное содержание работы. В следующей работе из этой серии мы дадим некоторые приложения этой теоремы. В частности, мы докажем, что в случае, когда $K$ - произвольное $\ell$-расширение некоторого абсолютно абелева числового поля $k$, для кругового $\mathbb{Z}_{\ell}$-расширения $K_{\infty} / K$ справедлива слабая гипотеза о $\ell$-адическом регуляторе. В [3] аналогичньй результат был доказан в предположении, что поле $K$ имеет абелевы пополнения $K_{v}$ для всех точек $v \mid \ell$.

Теперь мы кратко изложим идею доказательства основной теоремы и дадим обзор содержания работы. Наш метод состоит в том, чтобы обобшить на случай неабелевых локальных полей доказательство теоремы В из [7]. 
Начнем с вопроса о представлении логарифмов локальных единищ. Мы будем предполагать, что корни $\zeta_{n}$ согласованы для различных $n$ условиями $\zeta_{n+1}^{\ell}=\zeta_{n}$. Тогда в каждом круговом локальном поле $k_{n}$ имеется "хороший" локальный параметр $\pi_{n}=1-\zeta_{n}$ и группа $\log \left(U\left(k_{n}\right)\right)$ порождается логарифмами Артина-Хассе

$$
\eta_{n}(\alpha, a)=\sum_{r=0}^{\infty} \ell^{-r} \varphi^{r}(\alpha) \pi_{n}^{a \ell^{r}}
$$

где $\alpha$ принадлежит кольцу целых $\mathscr{O}_{H}$ поля $H, \varphi$ - автоморфизм $\Phi$ робениуса в $H$ и $a-$ некоторое натуральное число.

В $\S 1$ мы определяем набор локальных параметров $\lambda_{n} \in K_{n}$, которые ведут себя так же хорошо, как и $\pi_{n}$. Мы показываем, что существуют индекс $q$ и многочлен $F(X, T) \in \mathscr{O}_{H}[[T]][X]$, коэффициенты которого являются формальными степенными рядами от $T$, такие, что для всех $n \geqslant q$ в поле $K_{n}$ сушествует локальный параметр $\lambda_{n}$, являюшийся корнем уравнения $F^{(-n)}\left(X, \pi_{n}\right)=0$. Здесь и ниже $(-n)$ означает результат действия $\varphi^{-n}$ на коэффициенты рассматриваемых рядов. При этом $q$ можно определить как минимальный индекс такой, что $\nu_{\ell}\left(\mathscr{D}\left(K_{q} / k_{q}\right)\right)<1 / 2$, где $\mathscr{D}\left(K_{q} / k_{q}\right)$ - относительная дифферента и $\nu_{\ell}-\ell$-адический показатель такой, что $\nu_{\ell}(\ell)=1$.

Другой важный инвариант расширения $K_{\infty} / K$ - это натуральное число $\delta$, определяемое из условия $\left(\mathscr{D}\left(K_{q} / k_{q}\right)\right)=\left(\lambda_{q}^{\delta}\right)$. В то время как элементы $\pi_{n}$ удовлетворяют сравнению

$$
\pi_{n+1}^{\ell} \equiv \pi_{n} \quad(\bmod \ell)
$$

мы показываем, что элементы $\lambda_{n}$ могут быть выбраны так, что для всех $n \geqslant q$

$$
\lambda_{n+1}^{\ell} \equiv \lambda_{n} \quad\left(\bmod \ell \lambda_{n}^{-\delta}\right)
$$

что является хорошим приближением к (0.7).

В $\S 2$ мы рассматриваем выражение

$$
A:=\operatorname{Sp}_{K_{n} / \mathbb{Q}_{\ell}}\left(\left(\gamma_{p}-1\right) \Phi_{n}\left(\eta_{n}(\alpha, a)\right) \eta_{n}(\beta, b)\right)
$$

для некоторого $p \geqslant q$, где $\eta_{n}(\alpha, a)$ и $\eta_{n}(\beta, b)$ определены, как в $(0.6)$, с заменой $\pi_{n}$ на $\lambda_{n}$. Мы доказываем (теорема 2.1) некоторое сравнение по модулю $\mathbb{Z}_{\ell}$ для (0.9), позволяюшее представить $A$ как сумму выражений более простого вида, но распространенную на поля $K_{j}$ для всех $j \geqslant n$. Наши результаты здесь вполне аналогичны тем, что получены в [7]. Отличие только в том, что вместо (0.7) мы используем более слабое сравнение (0.8), поэтому в (0.9) мы вынуждены предполагать, что $p>q+2$ (при $\ell=2$ на $p$ накладьвается еще более сильное ограничение).

По сушеству, теорема 2.1 утверждает, что $A=\sum_{j=n}^{\infty} \operatorname{Sp}_{K_{j} / \mathbb{Q}_{\ell}}\left(R_{j}\left(\lambda_{j}\right)\right)$, где $R_{j}$ - некоторая функция от $\lambda_{j}$, слабо зависяшая от $j$. В круговом случае это означает, что существует формальный степенной ряд $R(T) \in \ell^{-m} \mathscr{O}_{H}[[T]]$ такой, что $R_{j}\left(\pi_{j}\right)=R^{(-j)}\left(\pi_{j}\right)$, т.е. зависимость $R_{j}$ от $j$ состоит просто в подкручивании коэффициентов ряда $R(T)$ с помощью $\varphi^{-j}$. Нечто похожее мы хотели бы сделать 
и в обшем случае, но здесь уже невозможно обойтись формальными степенными рядами. Приходится привлекать к рассмотрению формальные ряды Лорана (с коэффициентами в $\mathscr{O}_{H}$ ) с бесконечной главной частью.

В $\S 3$ мы даем нужные нам определения, относяшиеся к рядам Лорана, и доказываем первые нетривиальные результаты об этих рядах. Заметим, что в формулировку теоремы 2.1 входит величина

$$
S_{j}:=\mathscr{F}_{j}^{n-p-1}\left(\eta_{j}\left(\varphi^{n-j}(\alpha), a\right)\right)
$$

Проблема состоит в том, чтобы получить аналитическое выражение для $S_{j}$ как функции от $\lambda_{j}$. Для этого необходимо решить следуюшую задачу. Пусть $\sigma$-автоморфизм поля $K_{j}$ такой, что $\sigma\left(\zeta_{j}\right)=\zeta_{j} \xi_{\sigma}$ для некоторого корня из единицы $\xi_{\sigma}$. Как выразить аналитически $\sigma\left(\lambda_{j}\right)$ через $\lambda_{j}$ и $\xi_{\sigma}$ ? Мы знаем, что $\sigma\left(\lambda_{j}\right)$ является корнем уравнения

$$
F^{(-j)}\left(X, \sigma\left(\pi_{j}\right)\right)=0
$$

причем $\sigma\left(\pi_{j}\right)$ очевидным образом выражается аналитически через $\pi_{j}$ и $\xi_{\sigma}$, а $\lambda_{j}$ является приближенным решением (0.11) по модулю $1-\xi_{\sigma}$. Используя лемму Хензеля, мы показываем (теорема 3.1), что сушествует ряд Лорана от $\lambda: \mu(u, \lambda)$, коэффициенты которого являются формальными степенными рядами от $u$, где $\lambda$ и $u$ - независимые переменные, даюший в определенном смысле формальное решение уравнения (0.11). В частности, это означает, что $\sigma\left(\lambda_{j}\right)=\mu^{(-j)}\left(\xi_{\sigma}, \lambda_{j}\right)$ для всех $j \geqslant n$. Мы получаем также (см. (3.8)) оценки для коэффициентов ряда $\mu(u, \lambda)$ при отрицательных степенях $\lambda$, которые играют исключительно важную роль в дальнейшем.

В $\S 4$ мы доказываем (теорема 4.1), что существует формальный ряд Лорана $G(\lambda)$ с коэффициентами в $\mathscr{O}_{H}$ такой, что $S_{j}=G^{(-j)}\left(\lambda_{j}\right)$, причем снова нам удается проконтролировать поведение коэффициентов при отрицательных степенях $\lambda$.

Следует отметить, что ряд $G(\lambda)$ неудобен для дальнейшей работы, так как его коэффициенты при отрицательных степенях $\lambda$ слишком медленно убывают в $\ell$-адической метрике. В $\S 5$ мы исправляем этот недостаток. Заметим, что величина $S_{j}$ из $(0.10)$, являюшаяся функцией от $\lambda_{j}$, на самом деле лежит в поле $k_{j-s}$ и, следовательно, является функцией от $\lambda_{j-s}$, где $s=n-p-1$. В теореме 5.1 мы показываем, еще раз используя лемму Хензеля, что существует формальный ряд Лорана $\lambda_{\langle s\rangle}(\lambda)$, обладающий тем свойством, что $\lambda_{\langle s\rangle}^{(-j)}\left(\lambda_{j}\right)=\lambda_{j-s}$ для всех $j \geqslant n$. Мы доказываем (теорема 5.2), что существует ряд Лорана $D(X)$ такой, что $G(\lambda)=$ $D\left(\lambda_{\langle s\rangle}\right)$, откуда следует (теорема 5.3 ), что $S_{j}=D^{(-j)}\left(\lambda_{j-s}\right)$, причем $D(X)$ обладает быстро убывающими коэффициентами при отрицательных степенях $X$.

В $\S 6$ мы рассматриваем действие $\left(\gamma_{p}-1\right)$ на $S_{j}$ и соответственно на $D(X)$. Мы доказываем (теорема 6.3), что сушествует ряд Лорана $\mathscr{E}(X)$ такой, что для всех $j \geqslant n$

$$
\left(\gamma_{p}-1\right) S_{j}=\pi_{j-n \mathscr{E}^{(-j)}}\left(\lambda_{j-s}\right),
$$

причем $\mathscr{E}(X)$ снова обладает быстро убывающими коэффищиентами при отрица- 
В $\S 7$ мы заканчиваем доказательство основной теоремы. Напомним, что ряд $\mathscr{E}(X)$ строился для конкретного элемента $\eta_{n}(\alpha, a)$. Выберем $\eta_{n, 1}=\eta_{n}\left(\alpha_{1}, a_{1}\right), \ldots$

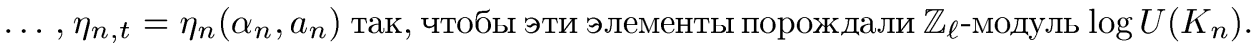
Тогда произвольный элемент $\eta \in \log U\left(K_{n}\right)$ записывается в виде линейной комбинации $\eta=\sum_{i=1}^{t} c_{i} \eta_{n, i}, c_{i} \in \mathbb{Z}_{\ell}$. При этом если $\mathscr{E}_{1}(X), \ldots, \mathscr{E}_{t}(X)-$ ряды, соответствующие $\eta_{n, 1}, \ldots, \eta_{n, t}$, то элементу $\eta$ сопоставляется ряд

$$
\mathscr{E}(X)=\mathscr{E}_{\eta}(X)=\sum_{i=1}^{t} c_{i} \mathscr{E}_{i}(X)
$$

Мы определим $M_{n}$ в формулировке основной теоремы как подмодуль, состояший из тех $\eta$, для которых ряд $\mathscr{E}_{\eta}(X)=\sum_{\nu=-\infty}^{\infty} e_{\nu} X^{\nu}$ в $(0.13)$ имеет нулевые коэффициенты $e_{\nu}$ для всех $\nu$ таких, что $|\nu| \leqslant 4(n-p+2) \ell \delta$. Тогда $M_{n}$ задается не более чем $8(n-p+2) \ell \delta f+f$ линейными уравнениями, где $f=\left[H: \mathbb{Q}_{\ell}\right]$, и мы можем положить в основной теореме $C=8 \ell \delta f$.

Пусть $\mathscr{E}_{\eta}(X)=\mathscr{E}_{1}(X)+\mathscr{E}_{2}(X)$, где $\mathscr{E}_{1}(X)$ содержит только степени $X^{\nu}$ для $\nu<-4(n-p+2) \ell \delta$, а $\mathscr{E}_{2}(X)$ содержит только степени $X^{\nu}$ с $\nu>4(n-p+2) \ell \delta$. Тогда для вычисления по модулю $\mathbb{Z}_{\ell}$ выражения $A:=\operatorname{Sp}_{K_{n} / \mathbb{Q}_{\ell}}\left(\left(\gamma_{p}-1\right) \Phi_{n}(\eta) \eta_{n, i}\right)$ для некоторого $\eta_{n, i}$ мы должны согласно теореме 2.1 исследовать выражение вида

$$
\ell^{-m-n+p-1} \sum_{j=n}^{\infty} \operatorname{Sp}_{K_{j} / \mathbb{Q}_{\ell}}\left(\pi_{j-n} \mathscr{E}^{(-j)}\left(\lambda_{j-s}\right) D^{(-j)}\left(\lambda_{j-s}\right)\right)
$$

где $\mathscr{E}(X)$ определен в $(0.13)$ и $D(X)$ - ряд, соответствующий элементу $\eta_{n, i}$. Мы представляем $D(X)$ в виде $D(X)=D_{1}(X)+D_{2}(X)$, где $D_{1}(X)$ может содержать степени $X^{\nu}$ только при $\nu<-4(n-p+2) \ell \delta$, а $D_{2}(X)$ может содержать степени $X^{\nu}$ только для $\nu \geqslant-4(n-p+2) \ell \delta$. Тогда $(0.14)$ содержит сумму произведений

$$
\mathscr{E}_{1}(X) D_{1}(X)+\mathscr{E}_{1}(X) D_{2}(X)+\mathscr{E}_{2}(X) D_{1}(X)+\mathscr{E}_{2}(X) D_{2}(X)
$$

Пользуясь нашими знаниями о поведении коэффициентов всех рассматриваемых рядов при отрицательных степенях $X$ (вот зачем мы все время отслеживали эти коэффициенты!), мы получаем, что первые три слагаемых не меняют (0.14) по модулю $\mathbb{Z}_{\ell}$. Таким образом, мы можем работать только со слагаемьм $\mathscr{E}_{2}(X) D_{2}(X)$, которое, как легко видеть, является формальным степенным рядом, т.е. не содержит отрицательных степеней $X$.

Мы показываем, что сушествует формальный степенной ряд $P(X)$ такой, что

$$
\operatorname{Sp}_{K_{j} / k_{j}}\left(\mathscr{E}_{2}^{(-j)}\left(\lambda_{j-s}\right) D_{2}^{(-j)}\left(\lambda_{j-s}\right)\right)=P^{(-j)}\left(\pi_{j-s}\right)
$$

что позволяет закончить доказательство основной теоремы теми же аргументами, что и в [7]. 


\section{§ 1. Система локальных параметров}

Пусть $K$ - произвольное конечное расширение поля $\mathbb{Q}_{\ell}$, содержашее первообразный корень из единицы степени $\ell$ (степени 4 при $\ell=2$ ). Для $n \geqslant 0$ обозначим через $\zeta_{n}$ первообразный корень из единицы степени $\ell^{m}$, где

$$
m=\left\{\begin{array}{lll}
n+1 & \text { при } & \ell \neq 2, \\
n+2 & \text { при } & \ell=2 .
\end{array}\right.
$$

Мы предполагаем, что корни $\zeta_{n}$ удовлетворяют условию $\zeta_{n+1}^{\ell}=\zeta_{n}$ при любом $n \geqslant 0$.

Пусть $K_{n}=K\left(\zeta_{n}\right)$ и $K_{\infty}=\bigcup_{n=0}^{\infty} K_{n}$ - круговое $\Gamma$-расширение поля $K$, где $\Gamma=G\left(K_{\infty} / K\right) \cong \mathbb{Z}_{\ell}$. Пусть $H$ - максимальное неразветвленное подполе поля $K_{\infty}$ и $\mathscr{O}_{H}-$ кольцо целых поля $H$. Тогда $H$ является конечным расширением $\mathbb{Q} \ell$. Положим $k_{n}=H\left(\zeta_{n}\right)$ и $k_{\infty}=\bigcup_{n=1}^{\infty} k_{n}$. Таким образом, $k_{\infty}$ - это максимальное круговое подполе поля $K_{\infty}$, и $K_{\infty} / k_{\infty}$ - конечное чисто разветвленное расширение, степень которого мы будем обозначать через $e$. Пусть $\nu_{\ell}()-\ell$-адический показатель, нормированный условием $\nu_{\ell}(\ell)=1$.

ПРЕДЛОЖЕНИЕ 1.1. Для всех достаточно больших $n \quad K_{n} / k_{n}$ является чисто разветвленным расширением степени е, не зависящей от $n$.

Пусть $\mathscr{D}\left(K_{n} / k_{n}\right)$ - дифферента расширения $K_{n} / k_{n}$. Тогда найдется индекс q такой, что

$$
\nu_{\ell}\left(\mathscr{D}\left(K_{n} / k_{n}\right)\right)<1 / 2 \quad \text { для всех } n \geqslant q .
$$

Действительно, согласно [8] мы имеем $\nu_{\ell}\left(\mathscr{D}\left(K_{n} / k_{n}\right)\right) \rightarrow 0$ при $n \rightarrow \infty$.

Для любого $n \geqslant 0$ элемент $\pi_{n}=1-\zeta_{n}$ является локальным параметром поля $k_{n}$. Наша ближайшая задача состоит в построении системы локальных параметров $\lambda_{n} \in K_{n}, n \geqslant q$, которые определенным образом согласованы друг с другом и с элементами $\pi_{n}$.

Рассмотрим кольцо формальных степенных рядов $R=\mathscr{O}_{H}[[T]]$. Пусть $\varphi-$ автоморфизм Фробениуса поля $H$. Мы продолжим действие $\varphi$ с $\mathscr{O}_{H}$ на $R$, полагая $\varphi(T)=T$. Если $f(T) \in R$, то ряд $f\left(\pi_{n}\right)$ сходится при любом $n \geqslant 0$, причем $(\varphi f)\left(\pi_{n}\right)=\varphi\left(f\left(\pi_{n}\right)\right)$, где в правой части равенства под $\varphi$ понимается автоморфизм поля $k_{n}$, совпадаюший с автоморфизмом Фробениуса на $H$ и такой, что $\varphi\left(\pi_{n}\right)=\pi_{n}$. Мы продолжим также действие $\varphi$ с $R$ на кольцо многочленов $R[X]$, полагая $\varphi(X)=X$. Для упрошения обозначений мы будем результат действия $\varphi^{i}$ на многочлен $F(X, T) \in R[X]$ обозначать просто через $F^{(i)}(X, T)$. Отметим, что любой многочлен $F(X, T) \in R[X]$ определяет для любого $n \geqslant 0$ многочлен $F\left(X, \pi_{n}\right) \in \mathscr{O}\left(k_{n}\right)[X]$, где $\mathscr{O}\left(k_{n}\right)$ - кольцо целых поля $k_{n}$.

ПРЕДЛОЖЕНИЕ 1.2. Пусть $q$ - индекс из предложения 1.1, m.е. $K_{n} / k_{n}-$ чисто разветвленное расширение степени е и $\nu_{\ell}\left(\mathscr{D}\left(K_{n} / k_{n}\right)\right)<1 / 2$ для всех

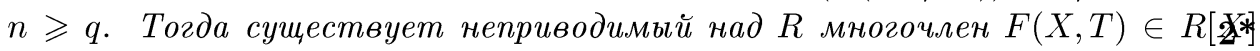


степени е со стариим коэффичиентом 1 такой, что многочлен $F\left(X, \pi_{n}\right)$ неприводим в $\mathscr{O}\left(k_{n}\right)[X]$ при любом $n \geqslant 0$, и при любом $n \geqslant q$ в поле $K_{n}$ существует локальный параметр $\lambda_{n}$, удовлетворяющий равенству $F^{(-n)}\left(\lambda_{n}, \pi_{n}\right)=0$. Существует иелое неотрицательное $\delta$, не зависящее от $n$ и такое, ито $\nu_{\ell}\left(\mathscr{D}\left(K_{n} / k_{n}\right)\right)=\nu_{\ell}\left(\lambda_{n}^{\delta}\right)$ для всех $n \geqslant q$. При әтом әлементы $\lambda_{n}$ можно вибрать так, чтобы при всех $n \geqslant q$ выполнялось сравнение

$$
\lambda_{n+1}^{\ell} \equiv \lambda_{n} \quad\left(\bmod \ell \lambda_{n}^{-\delta}\right) .
$$

ДоКАЗАТЕЛЬСТво. Пусть $\lambda_{q}$ - произвольный локальньй параметр поля $K_{q}$. Он является корнем минимального многочлена над полем $k_{q}$ вида

$$
F(X)=X^{e}+a_{e-1} X^{e-1}+\ldots+a_{0} \in \mathscr{O}\left(k_{q}\right)[X] .
$$

Так как $\lambda_{q}$ - локальный параметр в $K_{q}$ и расширение $K_{q} / k_{q}$ чисто разветвлено, то $F(X)$ является многочленом Эйзенштейна, т.е. коэффициенты $a_{i}$ делятся на $\pi_{q}$ при $0 \leqslant i \leqslant e-1$ и $a_{0}$ не делится на $\pi_{q}^{2}$. Мы можем записать коэффициенты $a_{i}$ в виде степенных рядов (или даже многочленов) от $\pi_{q}, a_{i}=f_{i}\left(\pi_{q}\right), 0 \leqslant i \leqslant e$, где $f_{i}(T) \in R$ и $f_{e}(T)=1$. Более того, при $0 \leqslant i \leqslant e-1$ ряд $f_{i}(T)$, если $a_{i} \neq 0$, может быть выбран в виде

$$
f_{i}(T)=T^{n_{i}} g_{i}(T),
$$

где $n_{i} \geqslant 1$ и $g_{i}(0) \not \equiv 0(\bmod \ell)$ при $0 \leqslant i \leqslant e-1$, причем $n_{0}=1$. Мы зафиксируем раз и навсегда некоторый выбор рядов $f_{i}(T)$, удовлетворяющих условию $(1.3)$, и рассмотрим многочлен $F(X, T) \in R[X]$, определенный условием

$$
F^{(-q)}(X, T)=X^{e}+f_{e-1}(T) X^{e-1}+\ldots+f_{0}(T) \in R[X] .
$$

Условие (1.3) показывает, что многочлен $F^{(-n)}\left(X, \pi_{n}\right)$ является многочленом Эйзенштейна при любом $n \geqslant 0$. Следовательно, для любого $n \geqslant 0$ многочлен $F^{(-n)}\left(X, \pi_{n}\right)$ неприводим и любой его корень $z \in \overline{\mathbb{Q}}_{\ell}$ удовлетворяет условию $\nu_{\ell}(z)=\nu_{\ell}\left(\pi_{n}\right) / e$.

ЛЕмма 1.1. Пусть $H(X, T)$ - формальная производная многочлена $F(X, T)$ по $X$ и $z_{n}$ - произвольный корень многочлена $F^{(-n)}\left(X, \pi_{n}\right)$. Тогда существует целое неотрицательное $\delta$, зависящее только от поля $K_{\infty}$ и такое, что

$$
\nu_{\ell}\left(H^{(-n)}\left(z_{n}, \pi_{n}\right)\right)=\delta \nu_{\ell}\left(z_{n}\right)=\frac{\delta}{e} \nu_{\ell}\left(\pi_{n}\right) \quad \text { для всех } n \geqslant q .
$$

ДокАЗАТЕЛЬСТво. Многочлен (от $\left.z_{n}\right) H^{(-n)}\left(z_{n}, \pi_{n}\right)$ является суммой одночленов вида

$$
i z_{n}^{i-1} f_{i}^{(-n)}\left(\pi_{n}\right), \quad 1 \leqslant i \leqslant e .
$$

Согласно (1.3) $f_{i}\left(\pi_{n}\right)=0$ тогда и только тогда, когда $f_{i}(T)=0$ в $R$. Ввиду равенства $\nu_{\ell}\left(\pi_{n}\right)=e \nu_{\ell}\left(z_{n}\right)$ все ненулевые одночлены в (1.5) имеют различные $\ell$-адические показатели, поэтому

$$
\nu_{\ell}\left(H^{(-n)}\left(z_{n}, \pi_{n}\right)\right)=\min \left\{\nu_{\ell}\left(i z_{n}^{i-1} f_{i}\left(\pi_{n}\right)\right)\right\},
$$

где минимум берется по всем $1 \leqslant i \leqslant e$ таким, что $f_{i}(T) \neq 0$. 
Пусть для $n=q$ указанный минимум достигается при $i=k$. Согласно нашим предположениям

$$
\begin{aligned}
\frac{1}{2}>\nu_{\ell}\left(\mathscr{D}\left(K_{q} / k_{q}\right)\right) & =\nu_{\ell}\left(H^{(-q)}\left(\lambda_{q}, \pi_{q}\right)\right)=\nu_{\ell}\left(k \lambda_{q}^{k-1} f_{k}\left(\pi_{q}\right)\right) \\
& =\nu_{\ell}(k)+(k-1) \nu_{\ell}\left(\lambda_{q}\right)+n_{k} \nu_{\ell}\left(\pi_{q}\right),
\end{aligned}
$$

откуда следует, что $k \not \equiv 0(\bmod \ell)$. Таким образом, $k$ определяется тем, что величина $(i-1)+n_{i} e$ достигает минимума при $i=k$, где $i$ пробегает все значения $1 \leqslant i \leqslant e$ такие, что $i \not \equiv 0(\bmod \ell)$ и $f_{i}(T) \neq 0$.

Заметим, что при любом $n \geqslant q$ мы имеем

$$
\nu_{\ell}\left(k z_{n}^{k-1} f_{k}\left(\pi_{n}\right)\right)=\nu_{\ell}(k)+\left(k-1+n_{k} e\right) \nu_{\ell}\left(z_{n}\right)<\frac{1}{2},
$$

поэтому минимум в (1.6) не может достигаться на индексе $k^{\prime} \equiv 0(\bmod \ell)$. Следовательно, он достигается на том же индексе $k$ при любом $n \geqslant q$. Полагая $\delta=k-1+n_{k} e$, мы получаем, что $\nu_{\ell}\left(H^{(-n)}\left(z_{n}, \pi_{n}\right)\right)=\delta \nu_{\ell}\left(z_{n}\right)$ для всех $n \geqslant q$, что доказывает лемму.

Мы будем строить систему локальных параметров $\left\{\lambda_{n}\right\}$ по индукции. Предположим, что элементы $\lambda_{q}, \ldots, \lambda_{n}, n \geqslant q$, удовлетворяющие нашему предложению, уже определены. Пусть $\lambda_{n}=y_{1}, \ldots, y_{e}$ и $z_{1}, \ldots, z_{e}-$ все корни многочленов $F^{(-n)}\left(X, \pi_{n}\right)$ и $F^{(-n-1)}\left(X, \pi_{n+1}\right)$ соответственно в некотором фиксированном алгебраическом замыкании $\overline{\mathbb{Q}}_{\ell}$ поля $\mathbb{Q}_{\ell}$. Мы покажем, что для любого $i=1, \ldots$, e cуществует единственный индекс $j=j(i)$ такой, что $k_{n+1}\left(z_{i}\right)=k_{n+1}\left(y_{j}\right)$ и $z_{i}^{\ell} \equiv y_{i}$ $\left(\bmod \ell y_{j}^{-\delta}\right)$. Действительно, возводя обе части равенства

$$
F^{(-n-1)}\left(z_{i}, \pi_{n+1}\right)=0
$$

в степень $\ell$ и учитывая, что

$$
\pi_{n+1}^{\ell}=\left(1-\zeta_{n+1}\right)^{\ell} \equiv 1-\zeta_{n}=\pi_{n} \quad(\bmod \ell)
$$

и $\varphi(a) \equiv a^{\ell}(\bmod \ell)$ для любого $a \in \mathscr{O}_{H}$, мы получаем

$$
0=\left(F^{(-n-1)}\left(z_{i}, \pi_{n+1}\right)\right)^{\ell} \equiv F^{(-n)}\left(z_{i}^{\ell}, \pi_{n}\right) \quad(\bmod \ell) .
$$

Таким образом, $z_{i}^{\ell}$ является приближенным (по модулю $\ell$ ) решением уравнения $F^{(-n)}\left(X, \pi_{n}\right)=0$ в поле $k_{n+1}\left(z_{i}\right)$. Применяя рассуждение леммы $1.1 \mathrm{k}$ вычислению производной $H^{(-n)}\left(X, \pi_{n}\right)$ многочлена $F^{(-n)}\left(X, \pi_{n}\right)$ в точке $X=z_{i}^{\ell}$, мы получаем

$$
\nu_{\ell}\left(H^{(-n)}\left(z_{i}^{\ell}, \pi_{n}\right)\right)=\delta \nu_{\ell}\left(z_{i}^{\ell}\right)=\frac{\delta}{e} \nu_{\ell}\left(\pi_{n}\right)=\delta \nu_{\ell}\left(\lambda_{n}\right)=\nu_{\ell}\left(H^{(-n)}\left(\lambda_{n}, \pi_{n}\right)\right)<\frac{1}{2} .
$$

Это означает, что согласно лемме Хензеля приближенное решение $z_{i}^{\ell}$ продолжается до некоторого решения $y_{j}$ уравнения $F^{(-n)}\left(X, \pi_{n}\right)=0$ такого, что

$$
z_{i}^{\ell} \equiv y_{j} \quad\left(\bmod \ell \lambda_{n}^{-\delta}\right) .
$$


Следовательно, $k_{n+1}\left(z_{i}\right) \supseteq k_{n+1}\left(y_{j}\right)$, а поскольку оба эти поля имеют степень $e$ над $k_{n+1}$, мы получаем, что $k_{n+1}\left(z_{i}\right)=k_{n+1}\left(y_{j}\right)$.

Теперь мы покажем, что (1.7) определяет некоторую подстановку $i \rightarrow j(i)$ множества $\{1, \ldots, e\}$, т.е. что соответствие $i \leftrightarrow j(i)$ корректно определено и взаимно однозначно на этом множестве. Предположим, что для некоторого $i$ элемент $j(i)$ определен неоднозначно, т.е. уравнение $F^{(-n)}\left(X, \pi_{n}\right)=0$ имеет два корня $y_{j}, y_{h}$, удовлетворяющие (1.7). Тогда

$$
\frac{1}{2}<\nu_{\ell}\left(y_{j}-y_{h}\right) \leqslant \sum_{\substack{k \neq j \\ 1 \leqslant k \leqslant e}} \nu_{\ell}\left(y_{j}-y_{k}\right)=\nu_{\ell}\left(\mathscr{D}\left(K_{n} / k_{n}\right)\right)<\frac{1}{2} .
$$

Противоречие.

Предположим теперь, что для $i \neq h$ мы имеем $j(i)=j(h)$. Согласно (1.7) это означает, что $z_{i}^{\ell} \equiv z_{h}^{\ell} \equiv y_{j}\left(\bmod \ell \lambda_{n}^{-\delta}\right)$, откуда, учитывая, что $\nu_{\ell}\left(\lambda_{n}^{\delta}\right)<1 / 2$, мы получаем $\nu_{\ell}\left(z_{i}-z_{h}\right)>1 /(2 \ell)$. Поскольку $n+1>q$, мы имеем, как и в $(1.8)$,

$$
\frac{1}{2 \ell}<\nu_{\ell}\left(z_{i}-z_{h}\right) \leqslant \nu_{\ell}\left(\mathscr{D}\left(K_{n+1} / k_{n+1}\right)\right)<\frac{1}{2 \ell},
$$

откуда следует инъективность, а значит, и сюръективность отображения $i \rightarrow j(i)$. Следовательно, для $\lambda_{n}=y_{1}$ найдется элемент $\lambda_{n+1}=z_{j(1)}$ такой, что $F^{(-n-1)}\left(\lambda_{n+1}, \pi_{n+1}\right)=0, \quad K_{n+1}=k_{n+1} K_{n}=k_{n+1}\left(\lambda_{n}\right)=k_{n+1}\left(\lambda_{n+1}\right)$ и $\lambda_{n+1}^{\ell} \equiv \lambda_{n}\left(\bmod \ell \lambda_{n}^{-\delta}\right)$. Этими условиями элемент $\lambda_{n+1}$ определяется однозначно. Из леммы 1.1 следует, что $\mathscr{D}\left(K_{n} / k_{n}\right)=\left(H^{(-n)}\left(\lambda_{n}, \pi_{n}\right)\right)=\lambda_{n}^{\delta}$ (другое доказательство этого равенства содержится в [9]). Предложение доказано.

Как обычно, для $g \in G\left(K_{n} / K_{q}\right)$ мы определим $i_{n}(g)$ как максимальный индекс $i$ такой, что $g(x) \equiv x\left(\bmod \lambda_{n}^{i}\right)$ для любого $x \in \mathscr{O}_{K_{n}}$.

ПРЕДЛОЖЕНИЕ 1.3. Пусть $n>p \geqslant q$, где q имеет тот же смысл, ито $u$ в предложении 1.1. Тогда

$$
\mathscr{D}\left(K_{n} / K_{p}\right)=\left(\ell^{n-p} \lambda_{n}^{\delta} \lambda_{p}^{-\delta}\right),
$$

где $\delta$ - константа из предложения 1.2 .

Пусть $\sigma-$ некоторая образующая группь $G\left(K_{n} / K_{p}\right)$. Тогда

$$
i_{n}(\sigma)=e \ell^{p+m-n}-\delta .
$$

ДокАЗАТЕЛЬСтво. Для доказательства (1.9) достаточно заметить, что в силу мультипликативного свойства дифференты мы имеем

$$
\mathscr{D}\left(K_{n} / K_{p}\right) \mathscr{D}\left(K_{p} / k_{p}\right)=\mathscr{D}\left(K_{n} / k_{n}\right) \mathscr{D}\left(k_{n} / k_{p}\right),
$$

и воспользоваться предложением 1.2. 
Для доказательства (1.10) заметим, что

$$
\nu_{\ell}\left(\mathscr{D}\left(K_{n} / K_{p}\right)\right)=\sum_{\substack{g \in G\left(K_{n} / K_{p}\right) \\ g \neq 1}} i_{n}(g) .
$$

Аналогичная формула имеет место и для $\nu_{\ell}\left(\mathscr{D}\left(K_{n} / K_{p+1}\right)\right)$. Вычитая ее из $(1.11)$ и используя (1.9), мы получаем

$$
\nu_{\ell}\left(\ell \lambda_{p}^{-\delta} \lambda_{p+1}^{\delta}\right)=\nu_{\ell}\left(\mathscr{D}\left(K_{n} / K_{p}\right)\right)-\nu_{\ell}\left(\mathscr{D}\left(K_{n} / K_{p+1}\right)\right)=\sum_{g \in V} i_{n}(g),
$$

где $V=G\left(K_{n} / K_{p}\right) \backslash G\left(K_{n} / K_{p+1}\right)$.

Множество $V$ состоит из $(\ell-1) \ell^{n-p-1}$ элементов, причем $g \in V$ тогда и только тогда, когда $g$ порождает $G\left(K_{n} / K_{p}\right)$. В частности, $i_{n}(g)$ имеет одно и то же значение для всех $g \in V$. Тогда, используя (1.12) и то, что $K_{n}$ имеет индекс ветвления $e(\ell-1) \ell^{m-1}$ над $\mathbb{Q} \ell$, мы получаем

$$
i_{n}(\sigma)=\frac{\nu_{\ell}\left(\ell \lambda_{p}^{-\delta} \lambda_{p+1}^{\delta}\right)}{(\ell-1) \ell^{n-p-1}} e(\ell-1) \ell^{m-1}=e \ell^{p+m-n}-\delta .
$$

Предложение доказано.

\section{§ 2. Редукция}

Пусть $q$ - индекс из предложения 1.1 и целые неотрицательные индексы $n, i$ таковы, что $n-i-1 \geqslant q$. Положим, как и в [7],

$$
\mathscr{F}_{n}^{(i)}=\ell \operatorname{Sp}_{\Gamma_{n-i} / \Gamma_{n}}-\operatorname{Sp}_{\Gamma_{n-i-1} / \Gamma_{n}} \in \mathbb{Q}_{\ell}\left[G\left(K_{n} / K_{q}\right)\right]
$$

где для любого $j \geqslant q \quad \Gamma_{j}=G\left(K_{\infty} / K_{j}\right)=G\left(k_{\infty} / k_{j}\right)$ и для любой конечной групшы $G \operatorname{Sp}_{G}=\sum_{\sigma \in G} \sigma \in \mathbb{Q}_{\ell}[G]$. Положим

$$
\Phi_{n}(q)=\ell^{-m-1} \sum_{i=0}^{n-q-1} \mathscr{F}_{n}^{(i)} .
$$

Для $p>q$ пусть $\gamma_{p}$ - некоторая фиксированная топологическая образуюшая группы $\Gamma_{p}$. Тогда определен элемент

$$
\Theta_{n}(q, p):=\left(\gamma_{p}-1\right) \Phi_{n}(q) \in \mathbb{Q}_{\ell}\left[G\left(K_{n} / K_{q}\right)\right]
$$

Заметим, что при $p \geqslant q$

$$
\Theta_{n}(q, p)=\left(\gamma_{p}-1\right) \ell^{-m-1} \sum_{i=0}^{n-p-1} \mathscr{F}_{n}^{(i)}=\Theta_{n}(p, p),
$$

поэтому вместо $\Theta_{n}(q, p)$ мы будем просто писать $\Theta_{n}(p)$. 
Пусть $\alpha \in \mathscr{O}_{H}$ и $a-$ некоторое натуральное число. Известно, что $\mathbb{Z}_{\ell \text {-модуль }}$ $\log \left(U\left(K_{n}\right)\right)$ порождается логарифмами Артина-Хассе

$$
\eta_{n}(\alpha, a)=\sum_{r=0}^{\infty} \ell^{-r} \varphi^{r}(\alpha) \lambda_{n}^{a \ell^{r}}
$$

где $\alpha$ пробегает некоторый $\mathbb{Z}_{\ell}$-базис $\mathscr{O}_{H}$ и $a$ пробегает все натуральные числа.

Нашей ближайшей целью является вычисление по модулю $\mathbb{Z}_{\ell}$ величины

$$
A:=\operatorname{Sp}_{K_{n} / \mathbb{Q}_{\ell}}\left(\Theta_{n}(p)\left(\eta_{n}(\alpha, a)\right) \eta_{n}(\beta, b)\right)
$$

для произвольных $\alpha, \beta \in \mathscr{O}_{H}$, натуральных $a, b$ и подходяшего $p \geqslant q$.

Для любых $x, y \in K_{n}$ мы имеем

$$
\operatorname{Sp}_{K_{n} / \mathbb{Q}_{\ell}}\left(\operatorname{Sp}_{K_{n} / K_{q}}(x)\left(\gamma_{p}-1\right)(y)\right)=\operatorname{Sp}_{K_{n} / \mathbb{Q}_{\ell}}\left(\left(\gamma_{p}^{-1}-1\right)\left(\operatorname{Sp}_{K_{n} / K_{q}}(x)\right) y\right)=0
$$

И

$$
\mathscr{F}_{n}^{(i)}(x)=\mathscr{F}_{n-i}^{(0)} \operatorname{Sp}_{K_{n} / K_{n-i}}(x) \in K_{n-i},
$$

откуда следует, что

$$
\operatorname{Sp}_{K_{n-i} / K_{n-i-1}}\left(\mathscr{F}_{n}^{(i)}(x)\right)=0, \quad \operatorname{Sp}_{K_{n} / \mathbb{Q}_{\ell}}\left(\mathscr{F}_{n}^{(i)}(x) \mathscr{F}_{n}^{(j)}(y)\right)=0
$$

при любых $0 \leqslant i, j \leqslant n-q-1, i \neq j$. Заметим, что для любого $y \in K_{n}$ сушествует представление

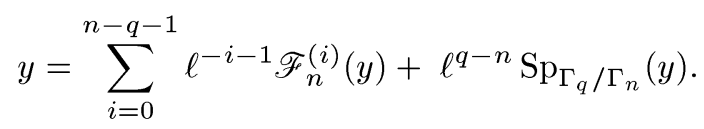

Поэтому для любых $x, y \in K_{n}$ мы имеем

$$
\operatorname{Sp}_{K_{n} / \mathbb{Q}_{\ell}}\left(\Theta_{n}(p)(x) y\right)=\ell^{-m-1} \operatorname{Sp}_{K_{n} / \mathbb{Q}_{\ell}}\left(\sum_{i=0}^{n-q-1} \ell^{-i-1}\left(\gamma_{p}-1\right) \mathscr{F}_{n}^{(i)}(x) \mathscr{F}_{n}^{(i)}(y)\right) .
$$

Применяя $(2.5)$ к $(2.2)$ и полагая $t_{n}(a, r)=\ell^{-r} \lambda_{n}^{a \ell^{r}}$, мы получаем

$$
\begin{aligned}
A=\operatorname{Sp}_{K_{n} / \mathbb{Q}_{\ell}\left[\sum_{i=0}^{n-q-1} \ell^{-m-i-2} \sum_{r, s=0}^{\infty}\left(\gamma_{p}-1\right) \mathscr{F}_{n}^{(i)}\right.}\left(\varphi^{r}(\alpha) t_{n}(a, r)\right) \\
\left.\times \mathscr{F}_{n}^{(i)}\left(\varphi^{s}(\beta) t_{n}(b, s)\right)\right] .
\end{aligned}
$$

Положим

$$
C_{n}^{i}(\alpha, a, r):=\mathscr{F}_{n}^{(i)}\left(\varphi^{r}(\alpha) t_{n}(a, r)\right) .
$$

Для $r<0$ мыполагаем по определению $C_{n}^{i}(\alpha, a, r)=0$. Тогда, считая $\alpha, \beta, a, b, p, q$ фиксированными, можно переписать (2.6) в виде

$$
A=\sum_{-\infty<r, s<\infty} B(r, s)
$$


где

$$
B(r, s):=\sum_{i=0}^{n-q-1} \ell^{-m-i-2} \operatorname{Sp}_{K_{n} / \mathbb{Q}_{\ell}}\left[\left(\gamma_{p}-1\right)\left(C_{n}^{i}(\alpha, a, r+i)\right) C_{n}^{i}(\beta, b, s+i)\right]
$$

ПРЕДЛОЖЕНИЕ 2.1. Пусть $n>p>q+2$. При $\ell=2$ пусть р удовлетворяет условию из лем.мы 2.2 (см. ниже). Тогда справедливо сравнение

$$
\begin{aligned}
B(r, s) \equiv \ell^{-m-n+q-1} \sum_{j=n}^{\infty} \operatorname{Sp}_{K_{j} / \mathbb{Q}_{\ell}}\left[\left(\gamma_{p}-1\right)\left(C_{j}^{n-q-1}\left(\varphi^{n-j}(\alpha), a, n+r-1\right)\right)\right. \\
\left.\times C_{j}^{n-q-1}\left(\varphi^{n-j}(\beta), b, n+s-1\right)\right] \bmod \mathbb{Z}_{\ell} .
\end{aligned}
$$

ДокАЗАТЕЛьство. Для доказательства нам потребуются две леммы.

Лемма 2.1. Пусть $n>q, n-q-1>i \geqslant 0, \alpha \in \mathscr{O}_{H}, a \geqslant 1 u r \geqslant 0$. Тогда справедливо сравнение

$$
C_{n}^{i}(\alpha, a, r) \equiv 0 \quad\left(\bmod \ell \lambda_{n-i-1}^{-2 \delta}\right)
$$

ДокАЗАТЕльство. Если $r=0$, то $\varphi^{r}(\alpha) t_{n}(a, r)$ - цельй элемент. Следовательно,

$$
\mathscr{F}_{n}^{(0)}\left(t_{n}(a, r)\right) \equiv \operatorname{Sp}_{K_{n} / K_{n-1}}\left(t_{n}(a, r)\right) \quad(\bmod \ell)
$$

Поскольку

$$
\mathscr{D}_{K_{n} / K_{n-1}}=\left(\ell \lambda_{n}^{\delta} \lambda_{n-1}^{-\delta}\right)
$$

мы имеем

$$
\mathscr{F}_{n}^{(0)}\left(t_{n}(a, r)\right) \equiv 0 \quad\left(\bmod \ell \lambda_{n-1}^{-\delta}\right)
$$

В силу (2.4)

$$
\mathscr{F}_{n}^{(i)}\left(t_{n}(a, r)\right)=\mathscr{F}_{n-i}^{(0)} \operatorname{Sp}_{K_{n} / K_{n-i}}\left(t_{n}(a, r)\right) \equiv 0 \quad\left(\bmod \ell \lambda_{n-1}^{-\delta}\right)
$$

Пусть теперь $r>0$. Тогда согласно (1.2) $\lambda_{n}^{\ell}=\lambda_{n-1}+\mu$, где $\mu \in K_{n}$ и $\mu \equiv 0$ $\left(\bmod \ell \lambda_{n-1}^{-\delta}\right)$. Возводя это равенство $r-1$ раз в степень $\ell$, мы получаем по индукции

$$
\lambda_{n}^{\ell^{r}} \equiv \lambda_{n-1}^{\ell^{r-1}}+\ell^{r-1} \mu \lambda_{n-1}^{\ell^{r-1}-1} \equiv \lambda_{n-1}^{\ell^{r-1}} \quad\left(\bmod \ell^{r} \lambda_{n-1}^{-\delta}\right)
$$

Таким образом,

$$
\varphi^{r}(\alpha) t_{n}(a, r)=\ell^{-1} \varphi^{r}(\alpha) t_{n-1}(a, r-1)+x
$$

для некоторого $x \in \lambda_{n-1}^{-\delta} \mathscr{O}_{K_{n}}$. Тогда из (2.7) следует, что при $i>0, r>0$

$$
C_{n}^{i}(\alpha, a, r)=C_{n-1}^{i-1}(\varphi(\alpha), a, r-1)+\mathscr{F}_{n}^{(i)}(x) .
$$


Из (2.9) вытекает, что $\operatorname{Sp}_{K_{n} / K_{n-1}}\left(\ell^{-1} \lambda_{n-1}^{\delta} \mathscr{O}_{K_{n}}\right) \subseteq \mathscr{O}_{K_{n-1}}$, поэтому $y:=$ $\operatorname{Sp}_{K_{n} / K_{n-1}}(x) \equiv 0\left(\bmod \ell \lambda_{n-1}^{-2 \delta}\right)$. Поэтому $\mathscr{F}_{n}^{(i)}(x)=\mathscr{F}_{n-1}^{(i-1)}(y)$ для некоторого $y \in K_{n-1}, y \equiv 0\left(\bmod \ell \lambda_{n-1}^{-2 \delta}\right)$.

Если $n-1>q$, то $\nu_{\ell}\left(\lambda_{n-1}^{2 \delta}\right) \leqslant \nu_{\ell}\left(\lambda_{q}^{2 \delta}\right)<1 / 2$, т.е. $\nu_{\ell}(y)>1 / 2$. Тогда $\mathscr{F}_{n-1}^{(i-1)}(y) \equiv 0(\bmod \ell)$, т.e.

$$
C_{n}^{i}(\alpha, a, r) \equiv C_{n-1}^{i-1}(\varphi(\alpha), a, r-1) \quad(\bmod \ell) .
$$

Пусть теперь $i$ и $r-$ любые индексы. Положим $c=\min \{i, r\}$. Если $c>0$, то, применяя (2.11) $c$ раз, мы придем к случаю, когда один из индексов $i, r$ равен нулю. Отметим, что по условию леммы $n-i-1>q$, поэтому условие $n-1>q$, при котором справедливо сравнение (2.11), будет все время соблюдаться.

Итак, достаточно рассмотреть случай, когда $i=0$ или $r=0$. Случай $r=0$ уже разобран. Если $i=0$ и $r>0$, то из (2.10) следует, что

$$
\mathscr{F}_{n}^{(0)}\left(\varphi^{r}(\alpha) t_{n}(a, r)\right)=\mathscr{F}_{n}^{(0)}\left(\varphi(\alpha) t_{n-1}(a, r-1)+x\right),
$$

где $x \in \lambda_{n-1}^{-\delta} \mathscr{O}_{K_{n}}$. Поскольку $\varphi^{r}(\alpha) t_{n-1}(a, r-1) \in K_{n-1}$, мы имеем $\mathscr{F}_{n}^{(0)}\left(\varphi^{r}(\alpha) \times\right.$ $\left.t_{n}(a, r-1)\right)=0$, т.е. $\mathscr{F}_{n}^{(0)}\left(\varphi^{r}(\alpha) t_{n}(a, r)\right)=\mathscr{F}_{n}^{(0)}(x)$. Тогда из $(2.9)$ следует, что $\mathscr{F}_{n}^{(0)}(x) \equiv 0\left(\bmod \ell \lambda_{n-1}^{-2 \delta}\right)$, что доказывает лемму.

ЛЕмма 2.2. При $\ell \neq 2$ для любых $n>q, \quad p>q, n-q-2>i \geqslant 0, a, b \geqslant 1$, $r, s \geqslant 0 \quad u \quad \alpha, \beta \in \mathscr{O}_{H}$ справедливо сравнение

$$
\begin{gathered}
\ell^{-m-i-2} \operatorname{Sp}_{K_{n} / \mathbb{Q}_{\ell}}\left[\left(\gamma_{p}-1\right)\left(C_{n}^{i}(\alpha, a, r)\right) C_{n}^{i}(\beta, b, s)\right] \\
\equiv \ell^{-m-i-3} \operatorname{Sp}_{K_{n+1} / \mathbb{Q}_{\ell}}\left[\left(\gamma_{p}-1\right)\left(C_{n+1}^{i+1}\left(\varphi^{-1}(\alpha), a, r+1\right)\right)\right. \\
\left.\times C_{n+1}^{i+1}\left(\varphi^{-1}(\beta), b, s+1\right)\right]\left(\bmod \mathbb{Z}_{\ell}\right) .
\end{gathered}
$$

При $\ell=2$ это сравнение справедливо, если $p \geqslant \log _{2}(9 \delta+1)-\log _{2} e-2$, где $\log _{2}$ - вещественный логарифм по основанию 2 .

ДокАЗАТЕЛЬСТво. Согласно (2.10) мы имеем

$$
C_{n+1}^{i+1}\left(\varphi^{-1}(\alpha), a, r+1\right)=\mathscr{F}_{n+1}^{(i+1)}\left(\ell^{-1} \varphi^{-1}(\alpha) t_{n}(a, r)+x\right)=C_{n}^{i}(\alpha, a, r)+a_{1},
$$

где $x \in \lambda_{n}^{-\delta} \mathscr{O}_{K_{n+1}}$ и $a_{1}=\mathscr{F}_{n+1}^{(i+1)}(x)$, в частности $a_{1} \in K_{n-i}$.

Из (1.9) следует, что $\mathscr{D}\left(K_{n+1} / K_{n-i}\right)=\ell^{i+1} \lambda_{n+1}^{\delta} \lambda_{n-i}^{-\delta}$. Учитывая, что $\nu_{\ell}\left(\lambda_{n}^{\delta}\right)<\nu_{\ell}\left(\lambda_{n-i}^{\delta}\right)$, мы получаем $\operatorname{Sp}_{K_{n+1} / K_{n-i-1}}(x) \equiv 0\left(\bmod \ell^{i+2} \lambda_{n-i-1}^{-2 \delta}\right)$. Аналогично, $\operatorname{Sp}_{K_{n+1} / K_{n-i}}(x) \equiv 0\left(\bmod \ell^{i+1} \lambda_{n-i}^{-2 \delta}\right)$. Следовательно, $a_{1} \equiv 0$ $\left(\bmod \ell^{i+2} \lambda_{n-i-1}^{-2 \delta}\right)$.

Аналогичным образом мы получаем, что

$$
C_{n+1}^{i+1}\left(\varphi^{-1}(\beta), b, s+1\right)=C_{n}^{i}(\beta, b, s)+a_{2},
$$


где $a_{2} \in K_{n-i}$ и $a_{2} \equiv 0\left(\bmod \ell^{i+2} \lambda_{n-i-1}^{-2 \delta}\right)$. Из (2.12) и (2.13) следует, что

$$
\begin{aligned}
& \ell^{-m-i-3} C_{n+1}^{i+1}\left(\varphi^{-1}(\alpha), a, r+1\right) C_{n+1}^{i+1}\left(\varphi^{-1}(\beta), b, s+1\right) \\
& =\ell^{-m-i-3} C_{n}^{i}(\alpha, a, r) C_{n}^{i}(\beta, b, s)+\ell^{-m-i-3} C_{n}^{i}(\alpha, a, r) a_{2} \\
& \quad+\ell^{-m-i-3} a_{1} C_{n}^{i}(\beta, b, s)+\ell^{-m-i-3} a_{1} a_{2} .
\end{aligned}
$$

Поскольку

$$
a_{1} \equiv a_{2} \equiv 0 \quad\left(\bmod \ell^{i+2} \lambda_{n-i-1}^{-2 \delta}\right)
$$

мы имеем

$$
a_{1} a_{2} \equiv 0 \quad\left(\bmod \ell^{2 i+4} \lambda_{n-i-1}^{-4 \delta}\right)
$$

поэтому

$$
\ell^{-m-i-3} a_{1} a_{2} \equiv 0 \quad\left(\bmod \ell^{-m+i+1} \lambda_{n-i-1}^{-4 \delta}\right) .
$$

Используя (2.15), лемму 2.1 и учитьвая, что $i \geqslant 0$, мы получаем

$$
\ell^{-m-i-3} C_{n}^{i}(\alpha, a, r) a_{2} \equiv \ell^{-m-i-3} a_{1} C_{n}^{i}(\beta, b, s) \equiv 0 \quad\left(\bmod \ell^{-m} \lambda_{n-i-1}^{-4 \delta}\right)
$$

Предположим теперь, что $\ell \neq 2$. В этом случае $\mathscr{D}\left(K_{n+1} / \mathbb{Q}_{\ell}\right)=\ell^{m+1} \pi_{0}^{-1} \lambda_{n+1}^{\delta}$, следовательно,

$$
\nu_{\ell}\left(\mathscr{D}\left(K_{n+1} / \mathbb{Q}_{\ell}\right)\right) \geqslant m+1-\frac{1}{\ell-1} .
$$

По условию леммы $n-i-1>q$, поэтому из условия $\nu_{\ell}\left(\lambda_{q}^{\delta}\right)<1 / 2$ вытекает, что $\nu_{\ell}\left(\lambda_{n-i-1}^{-4 \delta}\right)>-2 / \ell>-1$. Тогда из (2.16) следует, что $\ell^{-m-i-3} a_{1} a_{2} \equiv 0$ $\left(\bmod \ell^{-m}\right)$, т.е.

$$
\operatorname{Sp}_{K_{n+1} / \mathbb{Q}_{\ell}}\left(\ell^{-m-i-3} a_{1} a_{2}\right) \equiv 0 \quad\left(\bmod \mathbb{Z}_{\ell}\right)
$$

Аналогичным образом мы получаем $\nu_{\ell}\left(\lambda_{n-i-1}^{-4 \delta}\right)>-2 / \ell^{2} \geqslant-1 / 2$, поэтому оба члена в (2.17) сравнимы с нулем по модулю $\ell^{-m-1 / 2}$, т.е.

$$
\begin{aligned}
& \operatorname{Sp}_{K_{n+1} / \mathbb{Q}_{\ell}}\left(\ell^{-m-i-3} C_{n}^{i}(\alpha, a, r) a_{2}\right) \\
& \quad \equiv \operatorname{Sp}_{K_{n+1} / \mathbb{Q}_{\ell}}\left(\ell^{-m-i-3} a_{1} C_{n}^{i}(\beta, b, s)\right) \equiv 0\left(\bmod \mathbb{Z}_{\ell}\right)
\end{aligned}
$$

Следовательно,

$$
\begin{aligned}
& \operatorname{Sp}_{K_{n+1} / \mathbb{Q}_{\ell}}\left(\ell^{-m-i-3} C_{n+1}^{i+1}\left(\varphi^{-1}(\alpha), a, r+1\right) C_{n+1}^{i+1}\left(\varphi^{-1}(\beta), b, s+1\right)\right) \\
& \quad \equiv \operatorname{Sp}_{K_{n} / \mathbb{Q}_{\ell}}\left(\ell^{-m-i-2} C_{n}^{i}(\alpha, a, r) C_{n}^{i}(\beta, b, s)\right)\left(\bmod \mathbb{Z}_{\ell}\right) .
\end{aligned}
$$

Аналогичное рассуждение показывает, что

$$
\begin{aligned}
& \operatorname{Sp}_{K_{n+1} / \mathbb{Q}_{\ell}}\left(\ell^{-m-i-3} \gamma_{p}\left(C_{n+1}^{i+1}\left(\varphi^{-1}(\alpha), a, r+1\right)\right) C_{n+1}^{i+1}\left(\varphi^{-1}(\beta), b, s+1\right)\right) \\
& \equiv \operatorname{Sp}_{K_{n} / \mathbb{Q}_{\ell}}\left(\ell^{-m-i-2} \gamma_{p}\left(C_{n}^{i}(\alpha, a, r)\right) C_{n}^{i}(\beta, b, s)\right)\left(\bmod \mathbb{Z}_{\ell}\right)
\end{aligned}
$$

откуда следует справедливость леммы при $\ell \neq 2$. 
Пусть теперь $\ell=2$. В этом случае $\mathscr{D}\left(K_{n+1} / \mathbb{Q}_{\ell}\right)=\left(\ell^{m}\right)$. Снова мы имеем $\nu_{\ell}\left(\lambda_{n-i-1}^{-4 \delta}\right)>-1$, откуда следует, что сравнение (2.18) остается справедливым и при $\ell=2$.

Что касается сравнения (2.17), то при $\ell=2$ из него уже нельзя непосредственно вывести (2.19). Поэтому мы поступим следующим образом. Прежде всего заметим, что $C_{n}^{i}(\alpha, a, r), C_{n}^{i}(\beta, b, s), a_{1}, a_{2}$ являются элементами поля $K_{n-i}$. Для $x \in K_{n-i}$ положим $\operatorname{ord}(x)=e 2^{m-i-1} \nu_{\ell}(x)$, т.е. $\operatorname{ord}(x)$ - это показатель, с которым $\lambda_{n-i}$ входит в $x$. Без труда проверяется, что для любого $x \in K_{n-i}$ справедливо неравенство

$$
\operatorname{ord}\left(\gamma_{p}(x)-x\right) \geqslant \operatorname{ord}(x)+i\left(\gamma_{p}\right)-1,
$$

где в силу (1.10) $i\left(\gamma_{p}\right)=e 2^{p+2}-\delta$. Таким образом, при $p \geqslant \log _{2}(9 \delta+1)-\log _{2} e-2$ мы имеем $e 2^{p+2}-\delta-1 \geqslant 8 \delta$. Учитьвая, что $\operatorname{ord}\left(\lambda_{n-i-1}^{-4 \delta}\right)=-8 \delta$, мы получаем из $(2.17)$

$$
\begin{aligned}
& \ell^{-m-i-3}\left(\gamma_{p}-1\right)\left(C_{n}^{i}(\alpha, a, r)\right) a_{2} \\
& \quad \equiv \ell^{-m-i-3}\left(\gamma_{p}-1\right)\left(a_{1}\right) C_{n}^{i}(\beta, b, s) \equiv 0\left(\bmod \ell^{-m}\right)
\end{aligned}
$$

откуда следует, что для элементов, входящих в (2.20), выполняется аналог сравнения (2.19). После этого доказательство можно закончить так же, как и в случае $\ell \neq 2$. Этим наша лемма доказана полностью.

Лемма 2.3. Пусть $p>q+1,0 \leqslant i \leqslant n-p-1$ и хотя бы один из индексов $r+i, s+i$ отрицателен. При $\ell=2$ предположим дополнительно, что $p \geqslant \log _{2}(7 \delta+1)-\log _{2} e-2$. Пусть $c=-\min \{r+i, s+i\}$. Тогдa

$$
\begin{gathered}
\ell^{-m-i-c-2} \operatorname{Sp}_{K_{n+c} / \mathbb{Q}_{\ell}}\left[\left(\gamma_{p}-1\right)\left(C_{n+c}^{i+c}\left(\varphi^{-c}(\alpha), a, r+i+c\right)\right)\right. \\
\left.\times C_{n+c}^{i+c}\left(\varphi^{-c}(\beta), b, s+i+c\right)\right] \equiv 0\left(\bmod \mathbb{Z}_{\ell}\right) .
\end{gathered}
$$

ДоКАЗАТЕЛЬСТвО. По условию леммы хоть один из индексов $r+i+c, s+i+c$ равен нулю. Предположим, что $r+i+c=0$ и $s+i+c \geqslant 0$. Тогда

$$
C_{n+c}^{i+c}\left(\varphi^{-c}(\alpha), a, r+i+c\right)=\mathscr{F}_{n+c}^{(i+c)}\left(\varphi^{-c}(\alpha) t_{n+c}(a, r+i+c)\right)
$$

где $t_{n+c}(a, r+i+c)=t_{n+c}(a, 0)$ - целое число, откуда, используя явный вид дифференты $\mathscr{D}\left(K_{n+c} / K_{n-i-1}\right)$, мы получаем

$$
C_{n+c}^{i+c}\left(\varphi^{-c}(\alpha), a, r+i+c\right) \equiv 0 \quad\left(\bmod \ell^{i+c+1} \lambda_{n-i-1}^{-\delta}\right) .
$$

В силу леммы 2.1 мы имеем

$$
C_{n+c}^{i+c}\left(\varphi^{-c}(\beta), b, s+i+c\right) \equiv 0 \quad\left(\bmod \ell \lambda_{n-i-1}^{-2 \delta}\right) .
$$

Таким образом, левая часть (2.21) имеет вид

$$
\ell^{-m-i-c-2} \operatorname{Sp}_{K_{n+c} / \mathbb{Q}_{\ell}}\left(\left(\gamma_{p}-1\right)(x) y\right),
$$


где $x \equiv 0\left(\bmod \ell^{i+c+1} \lambda_{n-i-1}^{-\delta}\right)$ и $y \equiv 0\left(\bmod \ell \lambda_{n-i-1}^{-2 \delta}\right)$. Поэтому

$$
z:=\ell^{-m-i-c-2}\left(\gamma_{p}-1\right)(x) y \equiv 0 \quad\left(\bmod \ell^{-m} \lambda_{n-i-1}^{-3 \delta}\right)
$$

Если $\ell \neq 2$, то, учитывая, что $c \geqslant 1$, мы получаем, что $\mathscr{D}\left(K_{n+c} / \mathbb{Q}_{\ell}\right) \equiv 0$ $\left(\bmod \ell^{m+1} \pi_{0}^{-1}\right)$, т.е.

$$
\nu_{\ell}\left(\mathscr{D}\left(K_{n+c} / \mathbb{Q}_{\ell}\right)\right) \geqslant m+1-\frac{1}{\ell-1} .
$$

Из условия $i \leqslant n-p-1$ следует, что $n-i-1 \geqslant p>q+1$. Следовательно, $\nu_{\ell}\left(\lambda_{n-i-1}^{3 \delta}\right) \leqslant(1 / \ell) \nu_{\ell}\left(\lambda_{q+1}^{3 \delta}\right) \leqslant 1 / \ell$. Таким образом, мы получаем $\nu_{\ell}(z) \geqslant$ $\nu_{\ell}\left(\mathscr{D}\left(K_{n+c} / \mathbb{Q}_{\ell}\right)\right)$, т.е. $\operatorname{Sp}_{K_{n+c} / \mathbb{Q}_{\ell}}(z) \equiv 0\left(\bmod \mathbb{Z}_{\ell}\right)$.

Предположим теперь, что $\ell=2$. Тогда $\nu_{\ell}\left(\mathscr{D}\left(K_{n+c} / \mathbb{Q}_{\ell}\right)\right) \geqslant m$. Поскольку $x \in K_{n-i}$ и $x \equiv 0\left(\bmod \ell^{i+c+1} \lambda_{n-i}^{-2 \delta}\right)$, мы, используя $(1.10)$, получаем, что при $p \geqslant \log _{2}(7 \delta+1)-\log _{2} e-2$ справедливо сравнение $\left(\gamma_{p}-1\right)(x) \equiv 0\left(\bmod \ell^{i+c+1} \lambda_{n-i}^{4 \delta}\right)$, т.е. $z \equiv 0\left(\bmod \ell^{-m}\right)$ и $\operatorname{Sp}_{K_{n+c} / \mathbb{Q}_{\ell}}(z) \equiv 0\left(\bmod \mathbb{Z}_{\ell}\right)$.

Случай, когда $s+i+c=0$ и $r+i+c \geqslant 0$, разбирается аналогично. Лемма доказана.

ЛЕмма 2.4. Пусть $j \geqslant 2 n-p u r, s \geqslant 0$. При $\ell=2$ предположим дополнительно, что $p \geqslant \log _{2}(7 \delta+1)-\log _{2} e-2$. Тогда при любьх $\alpha, \beta \in \mathscr{O}_{H}$ и любих натуральных $a, b$

$$
\ell^{-m-n+p-1} \operatorname{Sp}_{K_{j} / \mathbb{Q}_{\ell}}\left[\left(\gamma_{p}-1\right)\left(C_{j}^{n-p-1}(\alpha, a, r)\right) C_{j}^{n-p-1}(\beta, b, s)\right] \equiv 0 \quad\left(\bmod \mathbb{Z}_{\ell}\right)
$$

ДокАЗАТЕЛЬСтво. Из леммы 2.1 следует, что

$$
u:=C_{j}^{n-p-1}(\alpha, a, r) C_{j}^{n-p-1}(\beta, b, s) \equiv 0 \quad\left(\bmod \ell^{2} \lambda_{j-n+p}^{-4 \delta}\right) .
$$

При $\ell \neq 2$, учитывая, что $j \geqslant 2 n-p$, мы имеем $\mathscr{D}\left(K_{j} / \mathbb{Q}_{\ell}\right) \equiv 0\left(\bmod \ell^{2 n-p+1} \pi_{0}^{-1}\right)$. Так как $\ell^{-m-n+p-1} u \equiv 0\left(\bmod \ell^{2 n-p} \lambda_{j-n+p}^{-4 \delta}\right)$, мы имеем

$$
\operatorname{Sp}_{K_{j} / \mathbb{Q}_{\ell}}\left(\ell^{-m-n+p-1} u\right) \equiv 0 \quad\left(\bmod \mathbb{Z}_{\ell}\right)
$$

Аналогичное рассуждение проходит и при $\ell=2$ и $j>2 n-p$.

Пусть теперь $\ell=2$ и $j=2 n-p$. Тогда $u \equiv 0\left(\bmod \ell^{2} \lambda_{n}^{-4 \delta}\right)$. Используя $(1.10)$, мы получаем, что в этом случае

$$
\left(\gamma_{p}-1\right)\left(C_{j}^{n-p-1}(\alpha, a, r)\right) C_{j}^{n-p-1}(\beta, b, s) \equiv 0 \quad\left(\bmod \ell^{2}\right)
$$

откуда следует справедливость леммы и в этом случае. Лемма доказана.

Теперь мы можем легко закончить доказательство предложения. Рассмотрим слагаемое

$$
V_{i}:=\ell^{-m-i-2} \operatorname{Sp}_{K_{n} / \mathbb{Q}_{\ell}}\left[\left(\gamma_{p}-1\right)\left(C_{n}^{i}(\alpha, a, r+i)\right) C_{n}^{i}(\beta, b, s+i)\right] .
$$


Если $r+i, s+i \geqslant 0$, то, применяя к $V_{i}$ лемму $2.2 n-p-1-i$ раз и полагая $j=2 n-p-1-i$, мы получаем

$$
\begin{aligned}
V_{i} \equiv & \ell^{-m-n+p-1} \operatorname{Sp}_{K_{j} / \mathbb{Q}_{\ell}}\left[\left(\gamma_{p}-1\right)\left(C_{j}^{n-p-1}\left(\varphi^{n-j}(\alpha), a, n+r-p-1\right)\right)\right. \\
& \left.\times C_{j}^{n-p-1}\left(\varphi^{n-j}(\beta), b, n+s-p-1\right)\right]\left(\bmod \mathbb{Z}_{\ell}\right) .
\end{aligned}
$$

Если один из индексов, например $r+i$, отрицателен, то $V_{i}=0$ по определению. В этом случае положим $c=-\min \{r+i, s+i\}$. Если $c>n-p-1-i$, то хотя бы один из индексов $n+r-p-1, n+s-p-1$ отрицателен. В этом случае сравнение (2.23) справедливо, поскольку обе его части равны нулю по определению.

Предположим теперь, что $c \leqslant n-p-1-i$. Тогда в силу леммы 2.3 выполняется сравнение (2.21). Применяя к его левой части лемму $2.2 n-p-i-c-1$ раз, мы получаем и в этом случае, что правая часть $(2.23)$ сравнима с нулем по модулю $\mathbb{Z}_{\ell}$.

Таким образом, сравнение (2.23) справедливо при $0 \leqslant i \leqslant n-p-1$ и любых целых $r, s$. Тогда из $(2.9)$ следует, что

$$
\begin{aligned}
B(r, s) \equiv & \ell^{-m-n+p-1} \\
& \times \sum_{j=n}^{2 n-p-1} \operatorname{Sp}_{K_{j} / \mathbb{Q}_{\ell}}\left[\left(\gamma_{p}-1\right)\left(C_{j}^{n-p-1}\left(\varphi^{n-j}(\alpha), a, n+r-p-1\right)\right)\right. \\
& \left.\times C_{j}^{n-p-1}\left(\varphi^{n-j}(\beta), b, n+s-p-1\right)\right]\left(\bmod \mathbb{Z}_{\ell}\right) .
\end{aligned}
$$

Из лемм 2.4 и 2.1 следует, что при $j \geqslant 2 n-p$ левая часть сравнения (2.22) целая и стремится к нулю при $j \rightarrow \infty$. Следовательно, сравнение (2.24) остается справедливым, если распространить суммирование на область $n \leqslant j<\infty$, что доказывает предложение.

Теорема 2.1. Пусть $n>p>q+2$. При $\ell=2$ предположим дополнительно, что $p \geqslant \log _{2}(9 \delta+1)-\log _{2} e-2$. Пусть $A-$ величина, определенная в $(2.2)$. Тогда

$$
\begin{aligned}
A \equiv \ell^{-m-n+p-1} \sum_{j=n}^{\infty} \operatorname{Sp}_{K_{j} / \mathbb{Q}_{\ell}}\left[\left(\gamma_{p}-1\right)\left(\mathscr{F}_{j}^{(n-p-1)}\left(\eta_{j}\left(\varphi^{n-j}(\alpha), a\right)\right)\right)\right. \\
\left.\quad \times \mathscr{F}_{j}^{(n-p-1)}\left(\eta_{j}\left(\varphi^{n-j}(\beta), b\right)\right)\right]\left(\bmod \mathbb{Z}_{\ell}\right) .
\end{aligned}
$$

ДокАЗАТЕЛЬСТВО. В силу предложения 2.1 мы имеем

$$
\begin{aligned}
A= & \sum_{-\infty<r, s<\infty} B(r, s) \\
\equiv & \sum_{-\infty<r, s<\infty} \sum_{j=n}^{\infty} \operatorname{Sp}_{K_{j} / \mathbb{Q}_{\ell}}\left[\left(\gamma_{p}-1\right)\left(C_{j}^{n-p-1}\left(\varphi^{n-j}(\alpha), a, n+r-p-1\right)\right)\right. \\
& \left.\times C_{j}^{n-p-1}\left(\varphi^{n-j}(\beta), b, n+s-p-1\right)\right]\left(\bmod \mathbb{Z}_{\ell}\right) .
\end{aligned}
$$


Меняя порядок суммирования, вводя новые индексы суммирования $r^{\prime}=n+r-$ $p-1, s^{\prime}=n+s-p-1$ и учитывая, что все слагаемые с $r^{\prime}<0$ или $s^{\prime}<0$ равны нулю, мы получаем

$$
\begin{aligned}
A=\ell^{-m-n+p-1} \sum_{j=n}^{\infty} \operatorname{Sp}_{K_{j} / \mathbb{Q}_{\ell}} \sum_{r^{\prime}, s^{\prime}=0}^{\infty}\left[\left(\gamma_{p}-1\right)\left(C_{j}^{n-p-1}\left(\varphi^{n-j}(\alpha), a, r^{\prime}\right)\right)\right. \\
\left.\times C_{j}^{n-p-1}\left(\varphi^{n-j}(\beta), b, s^{\prime}\right)\right] .
\end{aligned}
$$

Из (2.7) следует, что

$$
\left(\gamma_{p}-1\right) \sum_{r^{\prime}=0}^{\infty} C_{j}^{n-p-1}\left(\varphi^{n-j}(\alpha), a, r^{\prime}\right)=\left(\gamma_{p}-1\right) \mathscr{F}_{j}^{(n-p-1)}\left(\eta_{j}\left(\varphi^{n-j}(\alpha), a\right)\right)
$$

и

$$
\sum_{s^{\prime}=0}^{\infty} C_{j}^{n-p-1}\left(\varphi^{n-j}(\beta), b, s^{\prime}\right)=\mathscr{F}_{j}^{(n-p-1)}\left(\eta_{j}\left(\varphi^{n-j}(\beta), b\right)\right),
$$

что доказывает теорему.

\section{§ 3. Формальные ряды Лорана}

Хотя многочлен $F(X, T)$ из предложения 1.2 определен над кольцом $R=\mathscr{O}_{H}[[T]]$, некоторые вычисления, связанные с этим многочленом, требуют замены $R$ бо́льшим кольцом.

Пусть $\mathscr{O}$ означает одно из колец $\mathscr{O}_{H}, \mathscr{O}_{H}[\xi]$, где $\xi$ - первообразньй корень из единицы некоторой фиксированной степени $\ell^{k}$, или $\mathscr{O}_{H}[[u]]$, где $u-$ независимая переменная. Определим на $\mathscr{O}$ функцию $\nu$, которая на $\mathscr{O}_{H}$ и $\mathscr{O}_{H}[\xi]$ совпадает с $\ell$-адическим показателем $\nu_{\ell}$, а в случае $\mathscr{O}=\mathscr{O}_{H}[[u]]$, для одночлена $a u^{n}$, $a \in \mathscr{O}_{H}$, мы полагаем $\nu\left(a u^{n}\right)=\nu_{\ell}(a)+n$, а для $f(u)=\sum_{i=0}^{\infty} b_{i} u^{i}$ мы положим $\nu(f(u))=\inf _{i}\left\{\nu\left(b_{i} u^{i}\right)\right\}$. Мы будем рассматривать $\mathscr{O}_{H}[[u]]$ как топологическое кольцо относительно топологии, порожденной степенями максимального идеала $(\ell, u)$.

Пусть $\mathscr{A}=\mathscr{O}\{\{T\}\}$ - кольцо формальных рядов Лорана вида

$$
f(T)=\sum_{i=-\infty}^{\infty} a_{i} T^{i}, \quad a_{i} \in \mathscr{O}
$$

причем $\nu\left(a_{i}\right)>0$ при $i<0$ и $\nu\left(a_{i}\right) \rightarrow \infty$ при $i \rightarrow-\infty$. Без труда проверяется, что $\mathscr{A}$ не имеет делителей нуля. Для $f(T)$ вида $(3.1)$ мы полагаем $\nu(f)=$ $\min \left\{\nu\left(a_{i}\right)\right\}$, где минимум берется по всем $-\infty<i<\infty$.

ПРеДЛОЖеНИЕ 3.1. Ряд $f(T)$ вида (3.1) является единицей в $\mathscr{A}$ тогда и только тогда, когда а $a_{0}$ является единичей в $\mathscr{O}$. 
ДокАЗАТЕЛЬСТво. Необходимость условия очевидна. Если $a_{0} \in \mathscr{O}^{\times}$, то без ограничения общности можно считать, что $a_{0}=1$. В этом случае $f(T)$ представим в виде $f(T)=1+T f_{1}(T)+\pi f_{2}\left(T^{-1}\right)$, где $f_{1}(T) \in \mathscr{O}[[T]], f_{2}\left(T^{-1}\right) \in \mathscr{O}\left[\left[T^{-1}\right]\right]$, причем коэффициенты $b_{i}$ ряда $f_{2}\left(T^{-1}\right)$ удовлетворяют условию $\nu\left(b_{i}\right) \rightarrow 0$ при $i \rightarrow-\infty$ и $\pi=\ell, 1-\xi$ или $u$ соответственно. Тогда

$$
f(T)^{-1}=1+\sum_{n=1}^{\infty}\left(T f_{1}(T)+\pi f_{2}\left(T^{-1}\right)\right)^{n}
$$

Действительно, для заданного натурального $N$ и $n>2 N$ мы имеем $\left(T f_{1}(T)+\right.$ $\left.\pi f_{2}\left(T^{-1}\right)\right)^{n}=G_{1}+G_{2}$, где $G_{1} \in T^{N} \mathscr{O}[[T]]$ и $\nu\left(G_{2}\right) \geqslant \nu\left(\pi^{N}\right)$. Тогда ряд, стояший в правой части (3.2), сходится покоэффициентно и определяет в пределе ряд $f(T)^{-1} \in \mathscr{A}$, обратный к $f(T)$. Предложение доказано.

ПРЕДЛОЖЕНИЕ 3.2. Пусть $\mathscr{A}=\mathscr{O}\{\{T\}\}$, где $\mathscr{O}=\mathscr{O}_{H}$ или $\mathscr{O}=\mathscr{O}_{H}[\xi], u$ $\mathscr{K}$ - поле частных $\mathscr{A}$. Тогда $\mathscr{A}$ челозамкнуто в $\mathscr{K}$ и произвольный әлемент из $\mathscr{K}^{\times}$однозначно представим в виде $x=\pi^{n} T^{m} f(T)$, где $n, m \in \mathbb{Z}$, $f(T) \in \mathscr{A}^{\times}$и $\pi=\ell, 1-\xi-$ локальный параметр в $\mathscr{O}$.

ДокАЗАТЕЛЬСТво. Произвольный ряд $g_{1}(T) \in \mathscr{A}$ однозначно представим в виде $g_{1}(T)=\pi^{n_{1}} T^{m_{1}} f_{1}(T)$, где $n_{1} \geqslant 0, m_{1} \in \mathbb{Z}$, причем при $n_{1}=0$ выполняются условия: $m_{1} \geqslant 0$ и $f_{1}(T)$ - ряд, имеющий обратимьй свободный член и, следовательно, обратимый согласно предложению 3.1. Если $g_{2}(T)=\pi^{n_{2}} T^{m_{2}} f_{2}(T)-$ любой ненулевой элемент $\mathscr{A}$, то $g_{1}(T) / g_{2}(T)=\pi^{n_{1}-n_{2}} T^{m_{1}-m_{2}} f_{1}(T) f_{2}(T)^{-1}$, причем $f_{1}(T) / f_{2}(T) \in \mathscr{A}^{\times}$, что доказывает второе утверждение предложения. Предположим, что элемент $g_{1}(T)=\pi^{n} T^{m} f(T) \in \mathscr{K}$ цел над $\mathscr{A}$. Тогда без труда проверяется, что $n \geqslant 0$. Если $n>0$, то $g(T) \in \mathscr{A}$. Если $n=0$, то, редуцируя уравнение целой зависимости для $g(T)$ по модулю $\pi$, мы получаем, что $m \geqslant 0$, что полностью доказывает предложение.

Пусть $F(X)=F(X, T) \in R[X]-$ многочлен из предложения 1.2. Положим $\mathscr{B}=\mathscr{A}[X] /(F(X))$ и обозначим через $\lambda$ образ $X$ в $\mathscr{B}$.

ПрЕДЛОЖЕНИЕ 3.3. Кольцо Я̆В не имеет делителей нуля и естественно изоморфно кольиу $\mathscr{O}\{\{\lambda\}\}$.

ДокАЗАТЕЛЬСТво. Если $\mathscr{A}=\mathscr{O}\{\{T\}\}$, где $\mathscr{O}=\mathscr{O}_{H}$ или $\mathscr{O}=\mathscr{O}_{H}[\xi]$, и $\pi-$ локальный параметр в $\mathscr{O}$, то $F(X, T)(\bmod \pi)$ является многочленом Эйзенштейна той же степени над кольцом формальных степенных рядов $\mathscr{O} /(\pi)[[T]]$. Следовательно, $F(X, T)$ неприводим над $\mathscr{A}$. Для кольца $\mathscr{A}=\mathscr{O}_{H}[[u]]\{\{T\}\}$ неприводимость $F(X, T)$ над $\mathscr{A}$ следует из того, что $F(X, T)$ переходит в неприводимый многочлен той же степени при подстановке $u=0$. Следовательно, $\mathscr{B}$ - область целостности.

ЛЕмма 3.1. В кольще $\mathscr{O}_{H}[[\lambda]]$ существует однозначно определенный ряд $T=T(\lambda)$ такой, что $F(\lambda, T(\lambda))=0$. При этом $T(\lambda)=\lambda^{e} t(\lambda)$, әде $е-$ степень $F(X)$ ut $t(\lambda) \in \mathscr{O}_{H}[[\lambda]]^{\times}$. 
ДокАЗАТЕЛЬСТво. Согласно (1.3)

$$
F(X, T)=X^{e}+b_{e-1}(T)+\ldots+b_{1}(T) X+b_{0}(T)
$$

где $b_{i}(T) \in T R$ для $0 \leqslant i \leqslant e-1$ и $b_{0}(T)=T c(T)$ для некоторого $c(T) \in R^{\times}$. Положим $T_{1}(\lambda)=c(T)^{-1} \lambda^{e}$. Из $(3.3)$ следует, что $F\left(\lambda, T_{1}(\lambda)\right) \equiv 0\left(\bmod \lambda^{e+1}\right)$. Предположим, что для некоторого $i \geqslant 0$ уже определен ряд $T_{i}=T_{i}(\lambda)$ такой, что $F\left(\lambda, T_{i}(\lambda)\right) \equiv 0\left(\bmod \lambda^{e+i}\right)$. Тогда, полагая $T_{i+1}(\lambda)=T_{i}(\lambda)+x \lambda^{e+i}$ с неопределенным коэффициентом $x \in \mathbb{Z}_{\ell}$, мы получаем

$$
\begin{aligned}
F\left(\lambda, T_{i+1}(\lambda)\right) \equiv & \lambda^{e}+\sum_{n=1}^{e-1} b_{n}\left(T_{i}(\lambda)\right) \lambda^{n} \\
& +\left(T_{i}(\lambda)+x \lambda^{e+i}\right) c\left(T_{i}(\lambda)\right)\left(\bmod \lambda^{e+i+1}\right) .
\end{aligned}
$$

Поскольку $c(0) \in \mathbb{Z}_{\ell}^{\times}$, из (3.4) следует, что сушествует однозначно определенное по модулю $\ell$ число $x_{0} \in \mathbb{Z}_{\ell}$ такое, что при $x=x_{0} F\left(\lambda, T_{i+1}(\lambda)\right) \equiv 0\left(\bmod \lambda^{e+i+1}\right)$. Элементы $T_{i}(\lambda)$ при $i \rightarrow \infty$ сходятся к элементу $T(\lambda) \in \mathscr{O}_{H}[[\lambda]]$, удовлетворяюшему всем требованиям леммы. Лемма доказана.

Пусть $f(T)$ - произвольный элемент из $\mathscr{A}$. Без труда проверяется, что $f(T(\lambda))$ является корректно определенным элементом кольца $\mathscr{O}\{\{\lambda\}\}$ и сопоставление $f(T) \rightarrow f(T(\lambda))$ определяет вложение колец $\mathscr{A} \hookrightarrow \mathscr{O}\{\{\lambda\}\}$. Поскольку в $\mathscr{O}\{\{\lambda\}\}$ выполняется соотношение $f(\lambda, T(\lambda))=0$ и элементы $1, \lambda, \ldots, \lambda^{e-1}$ линейно независимы над $\mathscr{A}$, определенное выше вложение $\mathscr{A} \hookrightarrow \mathscr{O}\{\{\lambda\}\}$ продолжается до вложения $\mathscr{B} \hookrightarrow \mathscr{O}\{\{\lambda\}\}$. Чтобы доказать, что это вложение является изоморфизмом, достаточно проверить, что произвольный ряд $f(\lambda) \in \mathscr{O}\{\{\lambda\}\}$ является элементом $\mathscr{B}$.

Пусть $f(\lambda)=\sum_{j=-\infty}^{\infty} a_{i} \lambda^{i}$. Стандартные рассуждения показьвают, что формальньй степенной ряд $\sum_{i=0}^{\infty} a_{i} \lambda^{i}$ представим в виде

$$
\sum_{i=0}^{\infty} a_{i} \lambda^{i}=\sum_{j=0}^{e-1} \lambda^{j} f_{j}(T(\lambda)), \quad f_{j} \in \mathscr{O}[[T]] .
$$

Для $i<0$ член $a_{i} \lambda^{i}$ представим в виде $a_{i} \lambda^{i}=a_{i} \lambda^{j} \lambda^{-e k}$, где $j=0,1, \ldots, e-1$ и $k$ - некоторое натуральное число. Поскольку $T(\lambda)=\lambda^{e} t(\lambda)$, мы получаем $\lambda^{i}=\lambda^{j} T(\lambda)^{-k} t(\lambda)^{k}$. Согласно предыдущему замечанию ряд $\lambda^{j} t(\lambda)^{k}$ представим в виде (3.5), т.е.

$$
a_{i} \lambda^{i}=a_{i} T(\lambda)^{-k} \sum_{j=0}^{e-1} \lambda^{j} g_{i, j}(T(\lambda))
$$

для некоторых $g_{i, j} T(\lambda) \in \mathscr{O}[[T]]$. Поскольку $a_{i} \rightarrow 0$ при $i \rightarrow-\infty$, мы получаем

$$
f(\lambda)=\sum_{j=0}^{e-1} \lambda^{j}\left(f_{j}(T(\lambda))+\sum_{i=-1}^{\infty} a_{i} T(\lambda)^{-k} g_{i, j}(T(\lambda))\right),
$$


где $k=-[i / e]$ и $[i / e]-$ целая часть $i / e$, т.е. такое целое число, что $0 \leqslant i / e-[i / e]<1$ (напомним, что $i$ отрицательно), причем правая часть (3.6) является корректно определенным элементом кольца $\mathscr{B}$. Предложение доказано.

Начиная с этого места, мы будем обозначать кольца $\mathscr{O}_{H}\{\{T\}\}$ и $\mathscr{O}_{H}\{\{\lambda\}\}$ через $\mathscr{A}[\ell]$ и $\mathscr{B}[\ell]$, а $\mathscr{O}_{H}[\xi]\{\{T\}\}$ и $\mathscr{O}_{H}[\xi]\{\{\lambda\}\}$ через $\mathscr{A}[\xi]$ и $\mathscr{B}[\xi]$ соответственно. Кольца $\mathscr{O}_{H}[[u]]\{\{T\}\}$ и $\mathscr{O}_{H}[[u]]\{\{\lambda\}\}$ мы будем обозначать через $\widetilde{\mathscr{A}}$ и $\widetilde{\mathscr{B}}$ соответственно.

Мы определим в этих кольцах топологию, полагая, что для кольца вида $\mathscr{O}\{\{Y\}\}$ система

$$
\begin{aligned}
U_{m, n}:=\{ & f(Y) \in \mathscr{O}\{\{Y\}\} \mid f(Y)=f_{1}(Y)+f_{2}(Y), \\
& \left.f_{1}(Y), f_{2}(Y) \in \mathscr{O}\{\{Y\}\}, \quad \nu^{\prime}\left(f_{1}(Y)\right) \geqslant n, \quad f_{2}(Y) \equiv 0\left(\bmod Y^{m}\right)\right\}
\end{aligned}
$$

для $n, m \geqslant 0$ образует фундаментальную систему окрестностей нуля. Здесь $\nu^{\prime}=\nu_{\ell}$ для $\mathscr{O}=\mathscr{O}_{H}, \mathscr{O}_{H}[\xi]$ и $\nu^{\prime}=\nu$ для $\mathscr{O}=\mathscr{O}_{H}[[u]]$. Без труда проверяется, что все рассматриваемые кольца полны относительно этой топологии.

Пусть $G$ - циклическая группа порядка $\ell^{k}$ вместе с фиксированным изоморфизмом $G \cong\langle\xi\rangle$. Корень из единицы, соответствуюший элементу $\sigma \in G$ при этом изоморфизме, мы будем обозначать через $\xi_{\sigma}$.

ПРЕДЛОЖЕНИЕ 3.4. Существует единственное действие $G$ как группы непрерывных автоморфизмов кольца $\mathscr{A}[\xi]$ такое, что $G$ действует тождественно на $\mathscr{O}_{H}[\xi]$ и $\sigma(T)=1-\xi_{\sigma}+\xi_{\sigma} T$ для любого $\sigma \in G$.

ДоКАЗАТЕЛЬСТво. Очевидно, что определено действие $G$ на кольцо многочленов $\mathscr{O}_{H}[\xi][T]$, которое по непрерывности продолжается до действия $G$ на кольцо формальных степенных рядов $\mathscr{O}_{H}[\xi][[T]]$. Чтобыпродолжить это действие на $\mathscr{A}[\xi]$, заметим, что

$$
\sigma\left(T^{-1}\right)=\sigma(T)^{-1}=\left[\xi_{\sigma} T\left(1-\frac{1-\xi_{\sigma}^{-1}}{T}\right)\right]^{-1}=\xi_{\sigma}^{-1} T^{-1} \sum_{i=0}^{\infty}\left(1-\xi_{\sigma}^{-1}\right)^{i} T^{-i}
$$

Следовательно, полагая для произвольного $f(T) \in \mathscr{A}[\xi]$ вида $(3.1) \sigma(f(T))=$ $\sum_{i=-\infty}^{\infty} a_{i} \sigma\left(T^{i}\right)$, мы получаем непрерывный автоморфизм кольца $\mathscr{A}[\xi]$. Предложение доказано.

Для кольца $\tilde{\mathscr{A}}$ справедлив следующий аналог предложения 3.4 , который может быть доказан теми же рассуждениями.

ПРЕДЛОЖЕНИЕ 3.5. Существует единственный непрерывный автоморфизм $\tau$ кольиа $\widetilde{\mathscr{A}}$ такой, что $\tau$ действует тожественно на $\mathscr{O}_{H}[[u]] u$ $\tau(T)=u+(1-u) T=T+(1-T) u$.

Сопоставление $u \rightarrow 1-\xi_{\sigma}$ однозначно продолжается до гомоморфизма $f_{\sigma}: \tilde{\mathscr{A}} \rightarrow \mathscr{A}[\xi]$ mакого, ито $f_{\sigma} \tau=\sigma f_{\sigma}$. 
Tеорема 3.1. Пусть $\mathscr{F}(X)=\mathscr{F}(X, T)=\tau(F(X, T)) \in \widetilde{\mathscr{A}}[X]-$ многочлен, полученный действием $\tau$ на коэффичиенты многочлена (3.3). Тогда в кольце $\widetilde{\mathscr{B}}$ существует однозначно определенный ряд

$$
\mu=\mu(u, \lambda)=\sum_{j=-\infty}^{\infty} c_{j}(u) \lambda^{j}
$$

такой, ито $\mathscr{F}(\mu)=0 u \mu \equiv \lambda(\bmod u)$.

Коэффициенты $c_{j}(u) \in \mathscr{O}_{H}[[u]]$ для $j<0$ удовлетворяют условию

$$
\nu\left(c_{j}(u)\right)>-\frac{j}{2 \delta}
$$

где $\nu$ - определенная выше функция в кольце $\mathscr{O}_{H}[[u]]$ и $\delta$ имеет тот же смысл, что и в предложении 1.2 .

ДокАЗАТЕльство. Наша теорема является вариантом леммы Хензеля. Поскольку $\mathscr{F}(X) \equiv F(X, T)(\bmod u)$, мы имеем $\mathscr{F}(\lambda) \equiv 0(\bmod u)$. Наша задача заключается в том, чтобы поднять $\lambda$ до решения $\mu$ уравнения $\mathscr{F}(X)=0$. При этом мы вынуждены повторить все этапы доказательства леммы Хензеля, чтобы проконтролировать выполнение условия (3.8).

Пусть $H(X, T)$ - формальная производная многочлена $F(X, T)$ по $X$. Из доказательства леммы 1.1 и определения $\delta$ следует, что $H(\lambda, T(\lambda))=\lambda^{\delta} H_{1}(\lambda)+\ell H_{2}(\lambda)$, где $H_{1}(\lambda) \in \mathscr{O}_{H}[[\lambda]], H_{2}(\lambda) \in \lambda \mathscr{O}_{H}[[\lambda]]$ и $H_{1}(0) \not \equiv 0(\bmod \ell)$. Таким образом,

$$
H(\lambda, T(\lambda))=d_{0} \lambda^{\delta}\left(1-\sum_{i>-\delta}^{\infty} d_{i} \lambda^{i}\right)
$$

где $d_{0} \in \mathscr{O}_{H}^{\times}, d_{i} \in \mathscr{O}_{H}$ и $\nu_{\ell}\left(d_{i}\right) \geqslant 1$ при $i<0$. Следовательно,

$H(\lambda, T(\lambda))^{-1}=d_{0}^{-1} \lambda^{-\delta}\left(1+\sum_{n=1}^{\infty}\left(\sum_{i>-\delta}^{\infty} d_{i} \lambda^{i}\right)^{n}\right)=\lambda^{-\delta}\left(\sum_{i=-\infty}^{\infty} p_{i} \lambda^{i}\right)=\lambda^{-\delta} P(\lambda)$,

где $p_{i} \in \mathscr{O}_{H}, P(\lambda) \in \mathscr{B}[\ell]$ и для $i<0$ справедливо неравенство $\nu_{\ell}\left(p_{i}\right)>-i / \delta$.

Положим $\mu_{0}=\lambda$. Мы определим индуктивно последовательность приближений

$$
\mu_{i}=\sum_{j=-\infty}^{\infty} m_{i, j}(u) \lambda^{j} \in \widetilde{\mathscr{B}}
$$

такую, что $m_{i, j}(u)$ - многочлены от $u$ степени не выше $i$. Если $\mu_{i}$ уже определен для некоторого $i \geqslant 0$, то определим $\mu_{i+1}$ как ряд вида $(3.10)$, коэффициенты которого являются многочленами от $u$ степени не выше $i+1$ и который удовлетворяет сравнению

$$
\mu_{i+1} \equiv \mu_{i}-\mathscr{F}\left(\mu_{i}\right) / H(\lambda, T(\lambda)) \quad\left(\bmod u^{i+2}\right)
$$


Прежде всего мы покажем, что если $\mu_{i}$ уже определен и $\mathscr{F}\left(\mu_{i}\right) \equiv 0\left(\bmod u^{i+1}\right)$, то выполняются сравнения

$$
\begin{aligned}
\mu_{i+1} & \equiv \mu_{i}\left(\bmod u^{i+1}\right), \\
\mathscr{F}\left(\mu_{i+1}\right) & \equiv 0\left(\bmod u^{i+2}\right) .
\end{aligned}
$$

Действительно, по предположению индукции $\mathscr{F}\left(\mu_{i}\right) \equiv 0\left(\bmod u^{i+1}\right)$, поэтому $\mathscr{F}\left(\mu_{i}\right) / H(\lambda, T(\lambda)) \equiv 0\left(\bmod u^{i+1}\right)$. Тогда $(3.12)$ следует из $(3.11)$ и $(3.9)$.

Для доказательства (3.13) заметим, что для формальной переменной $Y$

$$
\mathscr{F}\left(\mu_{i}+Y\right)=\mathscr{F}\left(\mu_{i}\right)+\mathscr{F}_{1}\left(\mu_{i}\right) Y+\mathscr{F}_{2}\left(\mu_{i}\right) Y^{2}+\ldots+\mathscr{F}_{e}\left(\mu_{i}\right) Y^{e},
$$

где $\mathscr{F}_{1}\left(\mu_{i}\right)$ - значение производной многочлена $\mathscr{F}(X)$ в точке $X=\mu_{i}$ и $\mathscr{F}_{2}(X), \ldots$ $\ldots, \mathscr{F}_{e}(X)$ - многочлены от $X$ с коэффициентами из $\mathscr{O}_{H}[[u, \lambda]]$. Поскольку $\mu_{i} \equiv \lambda(\bmod u)$ и $\mathscr{F}(X) \equiv F(X)(\bmod u)$, мы имеем $\mathscr{F}_{1}\left(\mu_{i}\right)=H(\lambda, T(\lambda))+u G(\lambda)$ для некоторого $G(\lambda) \in \widetilde{\mathscr{B}}$. Тогда из $(3.9)$ следует, что $\mathscr{F}_{1}\left(\mu_{i}\right)=\lambda^{\delta} P(\lambda)^{-1}(1+$ $u P(\lambda) G(\lambda))$, откуда мы получаем, что $\mathscr{F}_{1}\left(\mu_{i}\right)^{-1} \equiv \lambda^{-\delta} P(\lambda) \equiv H(\lambda, T(\lambda))^{-1}$ $(\bmod u)$. Поскольку $\mathscr{F}\left(\mu_{i}\right) \equiv 0\left(\bmod u^{i+1}\right)$, мы получаем

$$
\mathscr{F}\left(\mu_{i}\right) / \mathscr{F}_{1}\left(\mu_{i}\right) \equiv \mathscr{F}\left(\mu_{i}\right) / H(\lambda, T(\lambda)) \quad\left(\bmod u^{i+2}\right) .
$$

Подставляя в $(3.14) Y=-\mathscr{F}\left(\mu_{i}\right) / \mathscr{F}_{1}\left(\mu_{i}\right)$ и учитывая (3.15), мы получаем (3.13).

В силу (3.12) существует однозначно определенный элемент $\mu \in \widetilde{\mathscr{B}}$ такой, что $\mu \equiv \mu_{i}\left(\bmod u^{i+1}\right)$ для всех $i \geqslant 0$. Из $(3.13)$ следует тогда, что $\mathscr{F}(\mu)=0$. Единственность $\mu$ следует из (3.14). Действительно, если $\mu^{\prime}-$ другой корень уравнения $\mathscr{F}(X)=0$ такой, что $\mu^{\prime} \equiv \lambda(\bmod u)$, то полагаем в $(3.14) \mu_{i}=\mu$ и $Y=\mu-\mu^{\prime}$. Противоречие.

Итак, нам осталось проверить выполнение условия (3.8). Вместо (3.8) мы рассмотрим более сильное условие, которое лучше подходит для применения индукции. Именно, мы будем доказывать, что при $j<0$ коэффициенты $c_{j}(u)$ удовлетворяют условию

$$
\nu\left(c_{j}(u)\right)>-\frac{1}{2}\left[\frac{j}{\delta}\right],
$$

где $[j / \delta]$ - такое целое число, что $0 \leqslant j / \delta-[j / \delta]<1$. Поскольку $c_{j}(u)=$ $\sum_{i=0}^{\infty} c_{i, j} u^{i}$, где $c_{i, j} \in \mathscr{O}_{H}$, для доказательства неравенства (3.16) достаточно проверить, что при заданном $i$ для всех $j<0$ вьполняется условие

$$
i+\nu_{\ell}\left(c_{i, j}\right)>-\frac{1}{2}\left[\frac{j}{\delta}\right]
$$

Отметим, что при $i+\nu_{\ell}\left(c_{i, j}\right)>0$ и $j \geqslant 0$ условие (3.17) всегда выполняется.

Мы докажем (3.17) индукцией по $i$. При $i=0$ мы имеем $\mu \equiv \lambda(\bmod u)$, т.е. $c_{0, j}=0$ при всех $j<0$. Следовательно, (3.17) вьполняется в этом случае тривиальным образом.

Предположим, что (3.17) уже доказано для всех $i<i_{0}$ и $j<0$ для некоторого индекса $i_{0} \geqslant 1$. Чтобы доказать, что (3.17) выполняется для $i=i_{0}$, мы заметим, 
что $\mu \equiv \mu_{i_{0}}\left(\bmod u^{i_{0}+1}\right)$ и $\mu_{i_{0}-1} \equiv \mu_{i_{0}}\left(\bmod u^{i_{0}}\right)$. Тогда из $(3.11)$ и $(3.12)$ следует, что

$$
\sum_{j=-\infty}^{\infty} c_{i_{0}, j} u^{i_{0}} \lambda^{j} \equiv \mathscr{F}\left(\mu_{i_{0}-1}\right) / H(\lambda, T(\lambda)) \quad\left(\bmod u^{i_{0}+1}\right) .
$$

Согласно (3.10) и (3.12)

$$
\mu_{i_{0}-1}=\sum_{j=-\infty}^{\infty} \sum_{k=0}^{i_{0}-1} c_{k, j} u^{k} \lambda^{j}, \quad c_{k, j} \in \mathscr{O}_{H}
$$

Пусть $q_{r}$ - коэффициент при $X^{r}$ многочлена $\mathscr{F}(X)$. Тогда

$$
q_{r}=b_{r}(\tau(T))=b_{r}(u+(1-u) T)=\sum_{s=0}^{\infty} u^{s} q_{r, s}(T(\lambda))=\sum_{s=0}^{\infty} u^{s} \sum_{t=0}^{\infty} q_{r, s, t} \lambda^{t}
$$

где $q_{r, s, t} \in \mathscr{O}_{H}$. Следовательно, $\mathscr{F}\left(\mu_{i_{0}-1}\right) / H(\lambda, T(\lambda))\left(\bmod u^{i_{0}+1}\right)$ является суммой одночленов вида

$$
u^{s} q_{r, s, t} \lambda^{t} \times \prod_{\alpha=1}^{r} c_{k_{\alpha}, j_{\alpha}} u^{k_{\alpha}} \lambda^{j_{\alpha}} \times \lambda^{-\delta} p_{i} \lambda^{i}
$$

где $s+k_{1}+\ldots+k_{r}=i_{0}$ и $p_{i}-$ коэффициенты из (3.9). Поэтому достаточно доказать, что условие вида (3.17) выполняется для любого одночлена (3.18).

Предположим, что $x_{1}=a_{1} u^{n_{1}} \lambda^{m_{1}}$ и $x_{2}=a_{2} u^{n_{2}} \lambda^{m_{2}}$, где $a_{1}, a_{2} \in \mathscr{O}_{H}$. Если $n_{1}+\nu_{\ell}\left(a_{1}\right)>-\left[m_{1} / \delta\right] / 2$ и $n_{2}+\nu_{\ell}\left(a_{2}\right)>-\left[m_{2} / \delta\right] / 2$, то

$$
n_{1}+n_{2}+\nu_{\ell}\left(a_{1} a_{2}\right)>-\frac{1}{2}\left[\frac{m_{1}}{\delta}\right]-\frac{1}{2}\left[\frac{m_{2}}{\delta}\right] \geqslant-\frac{1}{2}\left[\frac{m_{1}+m_{2}}{\delta}\right] .
$$

Таким образом, если для $x_{1}, x_{2}$ выполняется условие (3.17), то оно выполняется и для произведения $x_{1} x_{2}$. Если один из элементов $x_{1}, x_{2}$ удовлетворяет условию (3.17), а другой содержит $\lambda$ в неотрицательной степени, то $x_{1} x_{2}$ снова удовлетворяет условию (3.17). Поэтому наша задача сводится к тому, чтобы разбить каждое из произведений (3.18) на множители, каждый из которых либо удовлетворяет условию (3.17), либо содержит $\lambda$ в неотрищательной степени.

Если $s \geqslant 1$, то (3.18) после перестановки сомножителей преврашается в произведение вида

$$
u^{s} q_{r, s, t} \lambda^{t-\delta} \times \prod_{\alpha=1}^{r} c_{k_{\alpha}, j_{\alpha}} u^{k_{\alpha}} \lambda^{j_{\alpha}} \times p_{i} \lambda^{i} .
$$

Поскольку $t \geqslant 0$, мы имеем $s>-[(t-\delta) / \delta] / 2$. Любой из одночленов $c_{k_{\alpha}, j_{\alpha}} u^{k_{\alpha}} \lambda^{j_{\alpha}}$ входит в $\mu_{i_{0}-1}$, поэтому для него либо $j_{\alpha} \geqslant 0$, либо условие (3.17) выполнено по предположению индукции. Наконец, в $(3.9)$ мы имеем $\nu_{\ell}\left(p_{i}\right)>-i / \delta$. Поэтому $\nu_{\ell}\left(p_{i}\right)>-[i / \delta] / 2$. Следовательно, при $s \geqslant 1$ для одночлена (3.18) выполняется условие (3.17). Отметим, что при $i_{0}=1$ только этот случай и может встретиться. 
Рассмотрим теперь случай, когда $s=0$. Поскольку каждый из показателей $k_{\alpha}$ строго меньше $i_{0}$, мы имеем хотя бы пару положительных индексов $k_{\alpha}$. Пусть, например, $k_{1}, k_{2}>0$. В силу этого предположения, а также предположения индукции мы имеем

$$
2\left(\nu_{\ell}\left(c_{k_{1}, j_{1}}\right)+k_{1}\right)>-\left[\frac{j_{1}}{\delta}\right], \quad 2\left(\nu_{\ell}\left(c_{k_{2}, j_{2}}\right)+k_{2}\right)>-\left[\frac{j_{2}}{\delta}\right] .
$$

Поскольку в каждом из этих неравенств и справа, и слева стоят целые числа, мы получаем

$2\left(\nu_{\ell}\left(c_{k_{1}, j_{1}} c_{k_{2}, j_{2}}\right)+k_{1}+k_{2}\right)>-\left[\frac{j_{1}}{\delta}\right]-\left[\frac{j_{2}}{\delta}\right]+1 \geqslant-\left[\frac{j_{1}+j_{2}}{\delta}\right]+1=-\left[\frac{j_{1}+j_{2}-\delta}{\delta}\right]$,

т.е. для произведения $\lambda^{-\delta} c_{k_{1}, j_{1}} c_{k_{2}, j_{2}} u^{k_{1}+k_{2}} \lambda^{j_{1}+j_{2}}$ вьполняется условие (3.17). В силу этого в данном случае (3.18) можно представить в виде

$$
q_{r, 0, t} \lambda^{t} \times \lambda^{-\delta} \prod_{\alpha=1}^{2} c_{k_{\alpha}, j_{\alpha}} u^{k_{\alpha}} \lambda^{j_{\alpha}} \times \prod_{\alpha=3}^{r} c_{k_{\alpha}, j_{\alpha}} u^{k_{\alpha}} \lambda^{j_{\alpha}} \times p_{i} \lambda^{i},
$$

где произведение по $\alpha=3, \ldots, r$ предполагается равным 1 при $r=2$. Таким образом, мы разложили одночлен (3.18) в произведение сомножителей (отделенных друг от друга косым крестом), каждый из которых либо содержит $\lambda$ в неотрицательной степени, либо удовлетворяет условию (3.17), причем имеется хотя бы один такой сомножитель, а именно $\lambda^{-\delta} \prod_{\alpha=1}^{2} c_{k_{\alpha}, j_{\alpha}} u^{k_{\alpha}} \lambda^{j_{\alpha}}$, удовлетворяющий условию (3.17). Теорема доказана полностью.

Иэ доказанной теоремы вытекает следующий результат.

Теорема 3.2. Действие группь $G$ на кольцо $\mathscr{A}[\xi]$, определенное в предложении 3.4 , однозначно продолжается на кольчо $\mathscr{B}[\xi]$. Это продолжение однозначно определяется условием $\sigma(\lambda) \equiv \lambda\left(\bmod \left(1-\xi_{\sigma}\right)\right)$. При этом для любого $\sigma \in G$

$$
\sigma(\lambda)=\mu\left(1-\xi_{\sigma}, \lambda\right)=\sum_{j=-\infty}^{\infty} c_{j}\left(1-\xi_{\sigma}\right) \lambda^{j},
$$

где $c_{j}(u)$ - коэффициенты из (3.7).

ДокАЗАТЕЛЬСтво. Теорема 3.1 показывает, что автоморфизм $\tau$ кольца $\widetilde{\mathscr{A}}$ из предложения 3.5 однозначно продолжается до автоморфизма кольца $\widetilde{\mathscr{B}}$, которьй мы снова будем обозначать через $\tau$, такого, что $\tau(\lambda)=\mu$. Действительно, $\widetilde{\mathscr{B}} \cong \widetilde{\mathscr{A}}[X] /(F(X)) \cong \mathscr{O}_{H}[[u]]\{\{\lambda\}\}$, где $\lambda$ - корень многочлена $F(X)$, поэтому для любого гомоморфизма $\tau: \mathscr{A} \rightarrow \widetilde{\mathscr{A}}$, переводящего $F(X)$ в некоторый многочлен $\mathscr{F}(X)$, и любого корня $\mu$ многочлена $\mathscr{F}(X)$ сушествует единственное продолжение $\tau$ на кольцо $\widetilde{\mathscr{B}}$ такое, что $\tau(\lambda)=\mu$. То, что $\tau$ - автоморфизм, следует из того, что $\tau^{-1}-$ продолжение на $\widetilde{\mathscr{B}}$ автоморфизма $\tau^{-1}$ кольца $\mathscr{\mathscr { A }}$ такого, что $\tau^{-1}(T)=v+(1-v) T$, где $v=-\sum_{i=1}^{\infty} u^{i}$. При этом $\tau^{-1}(\lambda)=\mu(v, \lambda)$. Для $\sigma \in G$ пусть $\widetilde{f}_{\sigma}: \widetilde{\mathscr{B}} \rightarrow \mathscr{B}[\xi]$ - гомоморфизм колец, продолжаюший гомоморфизм $f_{\sigma}$ из 
предложения 3.5 , т.е. тождественньй на $\mathscr{O}_{H}$ и такой, что $\widetilde{f}_{\sigma}(\lambda)=\lambda$ и $\widetilde{f}_{\sigma}(u)=1-\xi_{\sigma}$. Тогда $\widetilde{f}_{\sigma}$ переводит $\mathscr{F}(X)$ в $\sigma(F(X))$ и корень $\mu=\mu(u, \lambda)$ многочлена $\mathscr{F}(X)$ в корень $\mu_{\sigma}=\mu\left(1-\xi_{\sigma}, \lambda\right)$ многочлена $\sigma(F(X))$. Отметим, что $\widetilde{f}_{\sigma}$ сюръективно только в том случае, когда $\sigma$ является образуюшей группы $G$.

Поскольку $\mathscr{B}[\xi] \cong \mathscr{A}[\xi][X] /(F(X))$, автоморфизм $\sigma$ кольца $\mathscr{A}[\xi]$ продолжается до автоморфизма $\sigma: \mathscr{B}[\xi] \rightarrow \mathscr{B}[\xi]$, при котором $\sigma(\lambda)=\mu\left(1-\xi_{\sigma}, \lambda\right)$. Следовательно, действие группы $G$ однозначно продолжается на $\mathscr{B}[\xi]$ и при этом выполняется соотношение $\widetilde{f}_{\sigma} \tau=\sigma \widetilde{f}_{\sigma}$ для любого $\sigma \in G$. Теорема доказана.

\section{§ 4. Применение формальных рядов Лорана}

В теореме 2.1 фигурирует выражение $S_{j}:=\mathscr{F}_{j}^{(n-p-1)}\left(\eta_{j}\left(\varphi^{n-j}(\alpha), a\right)\right)$, где $\eta_{j}\left(\varphi^{n-j}(\alpha), a\right)$ - логарифм Артина-Хассе, определенный в (2.1). Наша ближайшая цель состоит в том, чтобы доказать сушествование универсального формального ряда Лорана $G(\lambda) \in \mathscr{A}[\ell]=\mathscr{O}_{H}\{\{\lambda\}\}$, зависяшего от $n-p-1, \varphi^{n}(\alpha), a$ и такого, что для любого $j \geqslant n$ выполняется равенство $S_{j}=G^{(-j)}\left(\lambda_{j}\right)$, где $G^{(-j)}(\lambda)$ - ряд, полученный из $G(\lambda)$ действием $\varphi^{-j}$ на коэффициенты, и $\lambda_{j}-$ локальньй параметр поля $K_{j}$ из предложения 1.2 . В тех случаях, когда необходимо указать зависимость $G(\lambda)$ и $S_{j}$ от дополнительных параметров, мы будем писать $G\left(n-p-1, \varphi^{n}(\alpha), a, \lambda\right)$ и $S_{j}\left(n-p-1, \varphi^{n}(\alpha), a\right)$.

ПрЕДЛОЖЕНИЕ 4.1. Пусть $\sigma \in G\left(K_{j} / K_{j-n+p}\right) u \xi_{\sigma}-$ корень из единицы (не обязательно первовобразный) степени $\ell^{n-p}$, определенный условием $\sigma\left(\xi_{j}\right)=\xi_{\sigma} \zeta_{j}$. Пусть $\mu(u, \lambda) \in \mathscr{O}_{H}[[u]]\{\{\lambda\}\}-$ формальный ряд из (3.7) и $\mu^{(-j)}(u, \lambda)-$ ряд, полученный из $\mu(u, \lambda)$ действием $\varphi^{-j}$ на коэффициенты. Тогда ряд $\mu^{(-j)}\left(1-\xi_{\sigma}, \lambda_{j}\right)$ сходится в $K_{j}$ и справедливо равенство

$$
\sigma\left(\lambda_{j}\right)=\mu^{(-j)}\left(1-\xi_{\sigma}, \lambda_{j}\right) .
$$

ДокАЗАТЕльСтво. Действие автоморфизма Фробениуса $\varphi$, а также любой его целой степени на $\mathscr{O}_{H}$ естественно продолжается на $\mathscr{A}[\ell]$ и $\mathscr{A}[\xi]$. При этом мы считаем, что $\varphi(T)=T$ и $\varphi(\xi)=\xi$, где $\xi$ - первообразный корень из единицы степени $\ell^{n-p}$. Тогда $\varphi^{-j}$ индуцирует изоморфизм

$$
\begin{aligned}
\varphi^{-j}: \mathscr{B}[\xi] & =\mathscr{A}[\xi][X] /(F(X)) \\
& \cong \mathscr{B}^{(-j)}[\xi]:=\mathscr{A}[\xi][X] /\left(F^{(-j)}(X)\right) \cong \mathscr{A}[\xi]\left\{\left\{\lambda^{(-j)}\right\}\right\},
\end{aligned}
$$

который переводит $\lambda \in \mathscr{B}[\xi]$ в элемент $\lambda^{(-j)}$, являюшийся корнем многочлена $F^{(-j)}(X)$. При этом изоморфизме произвольный ряд $f(\lambda)=\sum_{i=-\infty}^{\infty} a_{i} \lambda^{i}$, $a_{i} \in \mathscr{O}_{H}[\xi]$, переходит в ряд

$$
f^{(-j)}\left(\lambda^{(-j)}\right)=\sum_{i=-\infty}^{\infty} \varphi^{-j}\left(a_{i}\right)\left(\lambda^{(-j)}\right)^{i} .
$$

Пусть $\hat{\sigma} \in G$ - такой автоморфизм кольца $\mathscr{B}[\xi]$, что $\hat{\sigma}(T)=\left(1-\xi_{\sigma}\right)+\xi_{\sigma} T$, где $\xi_{\sigma}$ имеет тот же смысл, что и в формулировке предложения. Применяя теорему 3.2 к кольцу $\mathscr{B}^{(-j)}[\xi]$ и многочлену $F^{(-j)}(X)$, мы получаем, что ограничение 
автоморфизма $\hat{\sigma}$ на $\mathscr{A}[\xi]$ однозначно продолжается до автоморфизма $\sigma_{1}$ кольца $\mathscr{B}^{(-j)}[\xi]$. Из однозначности этого продолжения следует, что $\varphi^{(-j)} \hat{\sigma}=\sigma_{1} \varphi^{(-j)}$. В частности, мы получаем, что $\mu^{(-j)}\left(1-\xi_{\sigma}, \lambda^{(-j)}\right)$ является корнем многочлена $\mathscr{F}^{(-j)}(X)=F^{(-j)}\left(X, 1-\xi_{\sigma}+\xi_{\sigma} T\right)$, т.е. что

$$
F^{(-j)}\left(\mu^{(-j)}\left(1-\xi_{\sigma}, \lambda^{(-j)}\right), 1-\xi_{\sigma}+\xi_{\sigma} T\right)=0 .
$$

Если $T=T(\lambda)$ - ряд из леммы 3.1 , то, применяя к обеим частям последнего равенства $\varphi^{-j}$, мы получаем $T=T^{(-j)}\left(\lambda^{(-j)}\right)$. Тогда (4.2) приводит к тождеству (в кольце $\mathscr{B}^{(-j)}[\xi]$ )

$$
F^{(-j)}\left(\mu^{(-j)}\left(1-\xi_{\sigma}, \lambda^{(-j)}\right), 1-\xi_{\sigma}+\xi_{\sigma} T^{(-j)}\left(\lambda^{(-j)}\right)\right)=0 .
$$

Из доказательства леммы 3.1 , примененного к многочлену $F^{(-j)}(X, T)$, следует, что $T^{(-j)}\left(\lambda_{j}\right)=\pi_{j}$. Легко показать, что ряд $\mu^{(-j)}\left(1-\xi_{\sigma}, \lambda_{j}\right)$ сходится в $K_{j}$ при $j \geqslant n$. Действительно, для коэффициентов этого ряда вьполнено условие (3.8). Если $\ell^{t}$ - порядок $\sigma$ в $G\left(K_{j} / K_{j-n+p}\right)$, то $\nu_{\ell}\left(1-\xi_{\sigma}\right)=\ell^{1-t}(\ell-1)^{-1}$, причем эта формула верна как при $\ell \neq 2$, так и при $\ell=2$. В любом случае $\nu_{\ell}\left(1-\xi_{\sigma}\right)>$ $\ell^{-t} \geqslant \ell^{p-n}$. Учитьвая, что любая степень $\varphi$ сохраняет $\ell$-адический показатель, мы получаем, что из условия $\nu\left(c_{r}(u)\right)=a$ следует, что $\nu_{\ell}\left(c_{r}^{(-j)}\left(1-\xi_{\sigma}\right)\right) \geqslant a \ell^{p-n}$. Согласно предложениям 1.1 и 1.2 мы имеем $\nu_{\ell}\left(\lambda_{q}^{\delta}\right)<1 / 2$. Поскольку $p>q$ и $j \geqslant n$, мы получаем, что $j-q>n-p$, т.е.

$$
\nu_{\ell}\left(\lambda_{j}^{\delta}\right)<\frac{1}{2} \ell^{p-n-1} .
$$

Это показывает, что член $c_{r}^{(-j)}\left(1-\xi_{\sigma}\right) \lambda_{j}^{r}$ является целым числом при $r<0$ и стремится к нулю в $K_{j}$ при $r \rightarrow-\infty$, т.е. ряд $\mu^{(-j)}\left(1-\xi_{\sigma}, \lambda^{(-j)}\right)$ сходится к некоторому $z \in \mathscr{O}_{K_{j}}$ такому, что $F^{(-j)}\left(z, \sigma\left(\pi_{j}\right)\right)=0$.

Так как $\mu(u, \lambda) \equiv \lambda(\bmod u)$, получаем из $(3.8)$, что $z \equiv \lambda_{j}\left(\bmod \left(1-\xi_{\sigma}\right) \lambda^{-2 \delta}\right)$, в частности $z \equiv \lambda_{j}\left(\bmod \lambda_{j}^{\delta+1}\right)$, причем $z$ - единственный корень многочлена $F^{(-j)}\left(X, \sigma\left(\pi_{j}\right)\right)$, удовлетворяющий последнему сравнению. Так как $\sigma\left(\lambda_{j}\right) \equiv \lambda_{j}$ $\left(\bmod \lambda_{j}^{i_{n}(\sigma)}\right)$ и $i_{n}(\sigma)$ определяется формулой $(1.10)$, мы получаем, что $\sigma\left(\lambda_{j}\right) \equiv \lambda_{j}$ $\left(\bmod \pi_{j}^{p+\alpha} \lambda_{j}^{-\delta}\right)$, где $\alpha=1$ при $\ell \neq 2$ и $\alpha=2$ при $\ell=2$. Поскольку $\nu_{\ell}\left(\pi_{j}\right) \geqslant$ $\ell^{-(j+1)}$, мы имеем $\nu_{\ell}\left(\pi_{j}^{p+\alpha}\right) \geqslant \ell^{p-j}$. Из неравенств $\nu_{\ell}\left(\lambda_{q}^{\delta}\right)<1 / 2$ и $p>q$ мы получаем $\nu_{\ell}\left(\lambda_{j}^{\delta}\right)<\ell^{p-j-1} / 2$, откуда следует, что $\sigma\left(\lambda_{j}\right) \equiv z\left(\bmod \lambda_{j}^{\delta+1}\right)$. Следовательно, $\sigma\left(\lambda_{j}\right)=z$. Предложение доказано.

Теорема 4.1. Существует формальный ряд Лорана $G(\lambda)=G(n-p-1$, $\left.\varphi^{n}(\alpha), a, \lambda\right) \in \mathscr{B}[\ell]$ такой, что для любого $j \geqslant n$ ряд $G^{(-j)}\left(\lambda_{j}\right)$ сходится $u$

$$
G^{(-j)}\left(\lambda_{j}\right)=\mathscr{F}_{j}^{(n-p-1)}\left(\eta_{j}\left(\varphi^{n-j}(\alpha), a\right)\right)
$$

Если $G(\lambda)=\sum_{i=-\infty}^{\infty} g_{i} \lambda^{i}$, mо $_{i} \in \ell_{\mathscr{O}_{H}}$ для всех $i$ и для всех $i<0$ справедливо неравенство

$$
\nu_{\ell}\left(g_{i}\right)>-\frac{i}{2 \delta \ell^{n-p}}
$$


ДокАЗАТЕЛЬСТво. Положим $S_{j}:=\mathscr{F}_{j}^{(n-p-1)}\left(\eta_{j}\left(\varphi^{n-j}(\alpha), a\right)\right)$ и

$$
S_{j, r}=\ell^{-r} \varphi^{n+r-j}(\alpha)\left[\ell \operatorname{Sp}_{K_{j} / K_{j-n+p+1}}\left(\lambda_{i}^{a \ell^{r}}\right)-\operatorname{Sp}_{K_{j} / K_{j-n+p}}\left(\lambda_{j}^{a \ell^{r}}\right)\right]
$$

Тогда $S_{j}=\sum_{r=0}^{\infty} S_{j, r}$.

Если $\sigma$ пробегает группу $G\left(K_{j} / K_{j-n+p+1}\right)$ (соответственно группу $\left.G\left(K_{j} / K_{j-n+p}\right)\right)$, то $\xi_{\sigma}$ пробегает все корни из единицы степени $\ell^{n-p-1}$ (соответственно степени $\left.\ell^{n-p}\right)$. Из предложения 4.1 непосредственно следует, что для любых $j \geqslant n$ и $r \geqslant 0$

$$
S_{j, r}=\ell^{-r} \varphi^{n+r-j}(\alpha) G_{r}^{(-i)}\left(\lambda_{j}\right),
$$

где формальньй ряд Лорана $G_{r}(\lambda) \in \mathscr{B}[\xi]$ определяется равенством

$$
G_{r}(\lambda)=\ell \sum_{\xi^{\ell^{n-p-1}}=1}(\mu(1-\xi, \lambda))^{a \ell^{r}}-\sum_{\xi^{\ell^{n-p}}=1}(\mu(1-\xi, \lambda))^{a \ell^{r}} .
$$

Покажем, что для любого $r \geqslant 0$ коэффициенты ряда $\ell^{-r} G_{r}(\lambda)$ принадлежат $\ell \mathscr{O}_{H}$ и удовлетворяют при $i<0$ неравенству вида (4.5). Затем покажем, что формальная сумма $\sum_{r \geqslant 0} \ell^{-r} \varphi^{n+r}(\alpha) G_{r}(\lambda)$ сходится покоэффициентно к некоторому ряду $G(\lambda) \in \mathscr{B}[\ell]$, удовлетворяющему всем условиям теоремы.

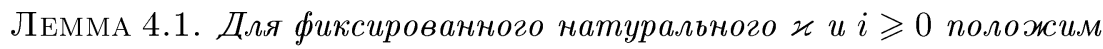

$$
d(i)=\ell \sum_{\xi^{\ell^{\varkappa}-1}=1}(1-\xi)^{i}-\sum_{\xi^{\ell^{\varkappa}}=1}(1-\xi)^{i} .
$$

Тогда $d(i) \in \ell^{\varkappa} \mathbb{Z}_{\ell} \partial$ $л я$ любого $i \geqslant 0 u d(0)=0$. Eсли $b:=\left[i \ell^{-\varkappa}\right]-$ челая часть $i \ell^{-\varkappa}$, то при любом $\ell$

$$
\nu_{\ell}(d(i)) \geqslant \varkappa+b .
$$

Eсли $\ell \neq 2$, mo

$$
\nu_{\ell}(d(i)) \geqslant \varkappa+b+b_{1}
$$

где $b_{1}=\left[i \ell^{-\varkappa-1}\right]$. Если $\ell=2$, mo

$$
\nu_{\ell}(d(i)) \geqslant \varkappa+b_{0}-1,
$$

əде $b_{0}=\left[i \ell^{1-\varkappa}\right]$.

ДокАЗАТЕЛЬСТво. Непосредственная проверка показывает, что

$$
\ell \sum_{\xi^{\varkappa-1}=1} \xi^{i}-\sum_{\xi^{\ell \varkappa}=1} \xi^{i}=\left\{\begin{aligned}
\ell^{\varkappa} & \text { при } i \equiv 0\left(\bmod \ell^{\varkappa-1}\right), \\
i & \neq 0\left(\bmod \ell^{\varkappa}\right), \\
0 & \text { в остальных случаях. }
\end{aligned}\right.
$$

Это доказывает, что $d(i) \in \ell^{\varkappa} \mathbb{Z}_{\ell}$ для всех $i$ и $d(0)=0$. 
Пусть $b=\left[i \ell^{-\varkappa}\right]>0$. Если $\xi_{\varkappa}$ - первообразный корень из единицы степени $\ell^{\varkappa}$, то $\nu_{\ell}\left(1-\xi_{\varkappa}\right)=\ell^{1-\varkappa} /(\ell-1)$, поэтому

$$
\left(1-\xi_{\varkappa}\right)^{i} \equiv 0 \quad\left(\bmod \ell^{b} \pi_{0}\right)
$$

где $\nu_{\ell}\left(\pi_{0}\right)=1 /(\ell-1)$. Положим $\alpha=1$ при $\ell \neq 2$ и $\alpha=2$ при $\ell=2$. Легко видеть, что при $i>0$

$$
\sum_{\xi^{\ell \varkappa}=1}(1-\xi)^{i}=\beta+\sum_{j=0}^{\varkappa-\alpha} \operatorname{Sp}_{k_{j} / \mathbb{Q}_{\ell}}\left(\left(1-\zeta_{j}\right)^{i}\right),
$$

где $\beta=0$ при $\ell \neq 2$ и $\beta=2^{i}$ при $\ell=2$. Поскольку для дифференты $\mathscr{D}\left(k_{\varkappa-\alpha} / \mathbb{Q} \ell\right)$ справедлива формула $\mathscr{D}\left(k_{\varkappa-\alpha} / \mathbb{Q} \ell\right)=\left(\ell^{\varkappa} \pi_{0}^{-1}\right)$, из $(4.11)$ следует, что

$$
\operatorname{Sp}_{k_{\varkappa-\alpha} / \mathbb{Q}_{\ell}}\left(\left(1-\zeta_{\varkappa-\alpha}\right)^{i}\right) \equiv 0 \quad\left(\bmod \ell^{\varkappa+b}\right)
$$

Учитывая, что $b \geqslant 1$, мы получаем из (4.11), что для любого $j=\varkappa-\alpha-c$, где $1 \leqslant c \leqslant \varkappa-\alpha$, справедливо сравнение

$$
\left(1-\zeta_{j}\right)^{i} \equiv\left(1-\zeta_{\varkappa-\alpha}\right)^{i \ell^{c}} \equiv 0 \quad\left(\bmod \ell^{b+c} \pi_{0}\right),
$$

откуда следует, что при любом $c$

$$
\operatorname{Sp}_{k_{j} / \mathbb{Q}_{\ell}}\left(\left(1-\zeta_{j}\right)^{i}\right) \equiv 0 \quad\left(\bmod \ell^{\varkappa+b}\right)
$$

Если $\ell=2$, то $\beta=2^{i} \equiv 0\left(\bmod \ell^{\varkappa+b}\right)$. Следовательно,

$$
\sum_{\xi^{\ell^{\varkappa}}=1}(1-\xi)^{i} \equiv 0 \quad\left(\bmod \ell^{\varkappa+b}\right)
$$

Аналогичные оценки показывают, что и

$$
\ell \sum_{\xi^{\varkappa-1}=1}(1-\xi)^{i} \equiv 0 \quad\left(\bmod \ell^{\varkappa+b}\right)
$$

что доказывает неравенство (4.8).

Если $\ell \neq 2$, то $\left(1-\xi_{\varkappa}\right)^{\ell^{\varkappa}} \equiv 0\left(\bmod \ell \pi_{0}\right)$, поэтому $\left(1-\xi_{\varkappa}\right)^{i} \equiv 0\left(\bmod \left(\ell \pi_{0}\right)^{b}\right)$, т.е. $\left(1-\xi_{\varkappa}\right)^{i} \equiv 0\left(\bmod \ell^{b+b_{1}} \pi_{0}\right)$, откуда, повторяя предыдущие рассуждения, мы получаем неравенство (4.9).

Если $\ell=2$, то $\left(1-\xi_{\varkappa}\right)^{\ell^{\varkappa-1}} \equiv 0(\bmod \ell)$, т.е. $\left(1-\xi_{\varkappa}\right)^{i} \equiv 0\left(\bmod \ell^{b_{0}}\right)$ и $\mathscr{D}\left(k_{\varkappa-\alpha} / \mathbb{Q} \ell\right)=\left(\ell^{\varkappa-1}\right)$, откуда легко следует неравенство (4.10). Лемма доказана.

ЗАмЕчАниЕ. Для произвольного $c \in \mathscr{O}_{H}$ положим $i_{0}:=\nu\left(c(1-\xi)^{i}\right)=\nu_{\ell}(c)+i$. Тогда

$$
\nu_{\ell}(c d(i)) \geqslant \varkappa+b
$$


где $b=\left[i_{0} \ell^{-\varkappa}\right]=\left[\left(\nu_{\ell}(c)+i\right) \ell^{-\varkappa}\right]$. Аналогичным образом обобшаются неравенства (4.9) и (4.10).

Действительно, пусть $\nu_{\ell}(c)=1$. В силу доказанной леммы $\nu_{\ell}(d(i+1)) \geqslant \varkappa+b$, где $b=\left[(i+1) \ell^{-\varkappa}\right]$. В доказательстве леммы было показано, что $\left(1-\xi_{\varkappa}\right)^{i+1} \equiv 0$ $\left(\bmod \ell^{b} \pi_{0}\right)$, поэтому $\left(1-\xi_{\varkappa}\right)^{i} \equiv 0\left(\bmod \ell^{b-1} \pi_{0}\right)$, откуда следует, что $\nu_{\ell}(d(i)) \geqslant$ $\varkappa+b-1$ и $\nu_{\ell}(c d(i)) \geqslant \varkappa+b$. Переходк произвольному $c$ осушествляется индукцией по $\nu_{\ell}(c)$, что доказывает (4.13). Обобщения неравенств (4.9) и (4.10) доказываются аналогично.

Мы применим лемму 4.1 и замечание к ней для оценок коэффициентов формального ряда $G_{r}(\lambda)$. Согласно теореме 3.1

$$
\mu(1-\xi, \lambda)=\lambda+\sum_{\substack{j=-\infty \\ j \neq 1}}^{\infty} c_{j}(1-\xi) \lambda^{j}=\lambda+\sum_{\substack{j=-\infty \\ j \neq 1}}^{\infty} \sum_{i=1}^{\infty} c_{i, j}(1-\xi)^{i} \lambda^{j}
$$

где $c_{j}(u) \in u \mathscr{O}_{H}[[u]]$ и $c_{i, j} \in \mathscr{O}_{H}$ для всех $i, j$. При этом согласно (3.8) $\nu\left(c_{j}(u)\right)>-j /(2 \delta)$, т.е. для любой пары индексов $i, j$ справедливо неравенство $\nu_{\ell}\left(c_{i, j}\right)+i>-j /(2 \delta)$.

Заметим, что для любых элементов $a_{1}, \ldots, a_{s}$ мы имеем

$$
\left(\sum_{i=1}^{s} a_{i}\right)^{\ell}=\sum_{i=1}^{s} a_{i}^{\ell}+\ell F\left(a_{1}, \ldots, a_{s}\right)
$$

где $F$ - некоторая форма степени $\ell$ от $a_{1}, \ldots, a_{s}$ с цельми рациональными коэффициентами. Применяя (4.15) $r$ раз к выражению $\left(\sum_{i=1}^{s} a_{i}\right)^{a}$, мы получаем

$$
\left(\sum_{i=1}^{s} a_{i}\right)^{a \ell^{r}}=\sum_{\alpha_{1}, \ldots, \alpha_{s}} b_{\alpha_{1}, \ldots, \alpha_{s}} a_{1}^{\alpha_{1}} \ldots a_{s}^{\alpha_{s}}
$$

где $\alpha_{1}, \ldots, \alpha_{s}$ пробегают все наборы целых неотрицательных чисел таких, что $\alpha_{1}+$ $\ldots+\alpha_{s}=a \ell^{r}$. При этом если $v=\nu_{\ell}\left(b_{\alpha_{1}, \ldots, \alpha_{s}}\right)$, то $\alpha_{1} \equiv \ldots \equiv \alpha_{s} \equiv 0\left(\bmod \ell^{r-v}\right)$.

Возводя правую часть (4.14) в степень $a \ell^{r}$ и используя (4.16), мы получаем, что ряд

$$
h(1-\xi, \lambda):=\mu(1-\xi, \lambda)^{a \ell^{r}}
$$

можно представить в виде сходящейся суммы одночленов вида $b c(1-\xi)^{i} \lambda^{j}$, где $b \in \mathbb{Z}$ - это коэффициент $b_{\alpha_{1}, \ldots, \alpha_{s}}$ для некоторого набора индексов $\alpha_{1}, \ldots, \alpha_{s}$ и $c(1-\xi)^{i} \lambda^{j}=\prod_{\nu=1}^{s}\left(c_{i_{\nu}, j_{\nu}}(1-\xi)^{i_{\nu}} \lambda^{j_{\nu}}\right)^{\alpha_{\nu}}$, причем $\sum_{\nu=1}^{s} \alpha_{\nu} i_{\nu}=i$ и $\sum_{\nu=1}^{s} \alpha_{\nu} j_{\nu}=j$. При этом из (4.16) следует, что для $v=\nu_{\ell}(b)$ справедливо сравнение

$$
\nu_{\ell}(c) \equiv i \equiv j \equiv 0 \quad\left(\bmod \ell^{r-v}\right),
$$

а из (3.8) следует, что

$$
\nu_{\ell}(c)+i>-\frac{j}{2 \delta}
$$


Тогда ряд $G_{r}(\lambda)$ можно представить в виде сходящейся суммы одночленов вида $b c d(i) \lambda^{j}$. Прежде всего мы покажем, что ряд $\ell^{-r} G_{r}(\lambda)$ имеет целые коэффициенты, делящиеся на $\ell$, для чего достаточно проверить, что для любого одночлена $b c(1-\xi)^{i} \lambda^{j}$, указанного вьше вида, справедливо неравенство $\nu_{\ell}(b c d(i))>r$. Если $n-p>r-v$, где $v=\nu_{\ell}(b)$, то согласно лемме $4.1 \nu_{\ell}(d(i)) \geqslant n-p$, т.е. $\nu_{\ell}(b c d(i)) \geqslant n-p+v>r$.

Предположим теперь, что $n-p \leqslant r-v$. Пусть $h=r-v-n+p$. Тогда из (4.17) следует, что $\left[i \ell^{p-n}\right]=\ell^{h}$ при $i \neq 0$, т.е. согласно (4.8) справедливо неравенство $\nu_{\ell}(b c d(i)) \geqslant v+n-p+\ell^{h}>r$. Этим доказано, что ряд $\ell^{-r} G_{r}(\lambda)$ имеет целые коэффициенты, деляшиеся на $\ell$.

Теперь мы покажем, что при $j<0$ для любого одночлена $b c(1-\xi)^{i} \lambda^{j}$, удовлетворяющего условиям (4.17) и (4.18), справедливо неравенство

$$
\nu_{\ell}\left(\ell^{-r} b c d(i)\right)>-\frac{j}{2 \delta \ell^{n-p}}
$$

Если $n-p>r-v$, где $v=\nu_{\ell}(b)$, то согласно (4.8), замечанию к лемме 4.1 и (4.18) мы имеем

$$
\nu_{\ell}(c d(i)) \geqslant n-p+\left[\left(\nu_{\ell}(c)+i\right) \ell^{p-n}\right] \geqslant n-p+\left[-\frac{j}{2 \delta \ell^{n-p}}\right] .
$$

Так как $-j /\left(2 \delta \ell^{n-p}\right)-\left[-j /\left(2 \delta \ell^{n-p}\right)\right]<1$ и $n-p-r+v \geqslant 1$, мы получаем (4.19).

Пусть $n-p=r-v$. Тогда $\left[\left(\nu_{\ell}(c)+i\right) \ell^{p-n}\right]=\left(\nu_{\ell}(c)+i\right) \ell^{p-n}$, и, снова используя (4.8) и (4.18), мы получаем

$$
\nu_{\ell}(c d(i)) \geqslant n-p+\left(\nu_{\ell}(c)+i\right) \ell^{p-n}>n-p-\frac{j}{2 \delta \ell^{n-p}}=r-v-\frac{j}{2 \delta \ell^{n-p}},
$$

т.е. (4.19) справедливо и в этом случае.

Если $n-p<r-v$, то мы рассмотрим по отдельности случаи $\ell \neq 2$ и $\ell=2$.

Если $\ell \neq 2$, то в силу $(4.17)$ при $j<0$ мы имеем

$$
\begin{aligned}
{\left[\left(\nu_{\ell}(c)+i\right) \ell^{p-n}\right] } & =\left(\nu_{\ell}(c)+i\right) \ell^{p-n} \geqslant \ell, \\
{\left[\left(\nu_{\ell}(c)+i\right) \ell^{p-n-1}\right] } & =\left(\nu_{\ell}(c)+i\right) \ell^{p-n-1} \\
& \geqslant \ell^{r-v+p-n-1} \geqslant r-v+p-n,
\end{aligned}
$$

поэтому из (4.9), замечания к лемме 4.1 и (4.18) следует, что

$$
\begin{aligned}
\nu_{\ell}(c d(i)) & \geqslant n-p+\left(\nu_{\ell}(c)+i\right) \ell^{p-n}-1 \\
& \geqslant n-p+\left(\nu_{\ell}(c)+i\right) \ell^{p-n}-1+r-v-n+p+1>r-v-\frac{j}{2 \delta \ell^{n-p}},
\end{aligned}
$$

откуда следует (4.19).

Если $\ell=2$ и $n-p<r-v$, то из (4.17) следует, что

$$
\begin{aligned}
{\left[\left(\nu_{\ell}(c)+i\right) \ell^{p-n+1}\right] } & =\left(\nu_{\ell}(c)+i\right) \ell^{p-n+1}=2\left(\nu_{\ell}(c)+i\right) \ell^{p-n} \\
& \geqslant\left(\nu_{\ell}(c)+i\right) \ell^{p-n}+\ell^{r-v-n+p} \\
& \geqslant\left(\nu_{\ell}(c)+i\right) \ell^{p-n}+r-v-n+p+1
\end{aligned}
$$


откуда, используя (4.10), замечание к лемме 4.1 и (4.18), мы получаем

$$
\nu_{\ell}(c d(i)) \geqslant n-p+\left(\nu_{\ell}(c)+i\right) \ell^{p-n+1}-1>r-v-\frac{j}{2 \delta \ell^{p-n}}
$$

что доказывает справедливость (4.19) и в этом случае.

Итак, мы доказали, что при любом $r$ коэффициенты ряда

$$
\ell^{-r} G_{r}(\lambda)=\sum_{j=-\infty}^{\infty} g_{r, j} \lambda^{j}
$$

обладают тем свойством, что $g_{r, j} \in \ell_{\mathscr{O}_{H}}$ при всех $j$, и для любого $j<0$ справедливо неравенство

$$
\nu_{\ell}\left(g_{r, j}\right)<-\frac{j}{2 \delta \ell^{n-p}}
$$

Теперь мы покажем, что формальная сумма $\sum_{r=0}^{\infty} \ell^{-r} \varphi^{n+r}(\alpha) G_{r}(\lambda)$ сходится покоэффициентно, т.е. что $\nu_{\ell}\left(g_{r, j}\right) \rightarrow \infty$ при $r \rightarrow \infty$ для любого фиксированного $j$. Как было показано выше, член $g_{r, j} \lambda^{j}$ ряда $\ell^{-r} G_{r}(\lambda)$ является суммой одночленов вида $\ell^{-r} b c d(i) \lambda^{j}$, удовлетворяюших условиям (4.17) и (4.18). Пусть $\gamma=\nu_{\ell}(j)$. Тогда из (4.17) следует, что $r-v \leqslant \gamma$, где $v=\nu_{\ell}(b)$, т.е.

$$
\nu_{\ell}\left(\ell^{-r} b\right) \leqslant \gamma
$$

Согласно (4.16) одночлен $\ell^{-r} b c d(i) \lambda^{j}$ имеет следующий вид. Существует некоторый набор целых неотрицательных чисел $\alpha_{1}, \ldots, \alpha_{s}$ и некоторый набор одночленов $c_{i_{\nu}, j_{\nu}}(1-\xi)^{i_{\nu}} \lambda^{j_{\nu}}, 1 \leqslant \nu \leqslant s$, входяших в (4.8), такой, что выполнены следуюшие условия:

(i) $-\infty<j<\infty$ и $i \geqslant 0$, причем из $i=0$ следует, что $j=1$ и $c_{0,1}=1$;

(ii) $\sum_{\nu=1}^{s} \alpha_{\nu}=a \ell^{r}$;

(iii) $\sum_{\nu=1}^{s} \alpha_{\nu} i_{\nu}=i$;

(iv) $\sum_{\nu=1}^{s} \alpha_{\nu} j_{\nu}=j$;

(v) $c=\prod_{\nu=1}^{s} c_{i_{\nu}, j_{\nu}}^{\alpha_{\nu}}$.

Нам нужно оценить величину коэффициента $c d(i)$ при $\lambda^{j}$, поэтому без ограничения общности мы можем предполагать, что все $\alpha_{\nu}$ положительны и что $j_{1}, \ldots, j_{t}<0, j_{t+1}, \ldots, j_{u}=0$ и $j_{u+1}, \ldots, j_{s}>0$. Тогда из (ii) следует, что хотя бы одна из сумм $S_{1}=\sum_{\nu=1}^{t} \alpha_{\nu}, S_{2}=\sum_{\nu=t+1}^{u} \alpha_{\nu}$ и $S_{3}=\sum_{\nu=u+1}^{s} \alpha_{\nu}$ не меньше, чем $a \ell^{r} / 3$.

Если $S_{1} \geqslant a \ell^{r} / 3$ или $S_{2} \geqslant a \ell^{r} / 3$, то, учитьвая, что $i_{\nu} \geqslant 1$ при $j_{\nu} \leqslant 0$, мы получаем из (iii), что $i \geqslant a \ell^{r} / 3$. Тогда из леммы 4.1 следует, что $\nu_{\ell}(d(i)) \geqslant$ $\left[a \ell^{r} /\left(3 \ell^{n-p}\right)\right]$, а это вместе с (4.20) дает нам, что $\nu_{\ell}\left(g_{r, j}\right) \rightarrow \infty$ при $r \rightarrow \infty$.

Если $S_{3} \geqslant a \ell^{r} / 3$, то $\sum_{\nu=u+1}^{s} \alpha_{\nu} j_{\nu} \geqslant a \ell^{r} / 3$, поэтому из (iv) следует, что

$$
-\sum_{\nu=1}^{t} \alpha_{\nu} j_{\nu} \geqslant \frac{a \ell^{r}}{3}-j
$$


Согласно (3.8) $\nu_{\ell}\left(c_{i_{\nu}, j_{\nu}}\right)+i_{\nu} \geqslant-j_{\nu} / 2 \delta$ для $\nu=1, \ldots, t$, откуда согласно $(\mathrm{v})$ следует, что

$$
\nu_{\ell}(c)+i \geqslant \sum_{\nu=1}^{t} \alpha_{\nu}\left(\nu_{\ell}\left(c_{i_{\nu}, j_{\nu}}\right)+i_{\nu}\right) \geqslant \frac{1}{2 \delta}\left(\frac{a \ell^{r}}{3}-j\right) .
$$

Тогда из (4.13) вытекает, что

$$
\nu_{\ell}(c d(i)) \geqslant n-p+\left[\left(\frac{a \ell^{r}}{3}-j\right) \frac{1}{2 \delta \ell^{n-p}}\right]
$$

т.е. и в этом случае $\nu_{\ell}\left(g_{r, j}\right) \rightarrow \infty$ при $r \rightarrow \infty$. Следовательно, формальная сумма $G(\lambda):=\sum_{r=0}^{\infty} \ell^{-r} \varphi^{n+r}(\alpha) G_{r}(\lambda)$ сходится покоэффициентно и определяет формальньй ряд Лорана $G(\lambda)$, коэффициенты которого $g_{i}$ принадлежат $\ell \mathscr{O}_{H}$ и удовлетворяют условию (4.5). Из этого условия следует, что $G(x)$ сходится для любого $x \in \overline{\mathbb{Q}}_{\ell}$ такого, что

$$
0<\nu_{\ell}(x)<\left(2 \delta \ell^{n-p}\right)^{-1} .
$$

Более того, легко видеть, что для такого $x$

$$
G(x)=\sum_{r=0}^{\infty} \ell^{-r} \varphi^{n+r}(\alpha) G_{r}(x) .
$$

Действительно, нам достаточно проверить, что $\nu_{\ell}\left(\ell^{-r} G_{r}(x)\right) \rightarrow \infty$ при $r \rightarrow \infty$. Пусть $N$ - достаточно большое натуральное число. Мы имеем

$$
\ell^{-r} G_{r}(x)=\sum_{j=-\infty}^{\infty} g_{r, j} \lambda^{j}=\sum_{j=N+1}^{\infty} g_{r, j} \lambda^{j}+\sum_{j=-N}^{N} g_{r, j} \lambda^{j}+\sum_{j=-\infty}^{-N-1} g_{r, j} \lambda^{j} .
$$

Обозначим эти три суммы через $S_{1}, S_{2}$ и $S_{3}$ соответственно. Тогда $\nu_{\ell}\left(S_{1}\right) \geqslant$ $N \nu_{\ell}(x)$, показатель $\nu_{\ell}\left(S_{2}\right)$ при фиксированном $N$ и $r \rightarrow \infty$ становится сколь угодно большим, так как $\nu_{\ell}\left(g_{r, j}\right) \rightarrow \infty$ при $r \rightarrow \infty$ для любого $j$. Наконец, в силу (4.5) мы имеем

$$
\nu_{\ell}\left(S_{3}\right) \geqslant N\left(\frac{1}{2 \delta \ell^{n-p}}-\nu_{\ell}(x)\right) .
$$

Этим доказано, что для $x$, удовлетворяющего (4.21), справедлива формула (4.22). В частности, действуя на коэффициенты в обеих частях формулы (4.22) автоморфизмом $\varphi^{-j}$, подставляя $x=\lambda_{j}$, где $j \geqslant n$, и используя (4.6), мы получаем (4.4). Теорема доказана полностью.

\section{§ 5. $G$-инвариантные ряды Лорана}

В $\S 3$ было определено действие циклической групшы $G$ порядка $\ell^{k}$ на кольца $\mathscr{A}[\xi]$ и $\mathscr{B}[\xi]$. В этом параграфе для произвольной подгруппы $\mathscr{H} \subseteq G$ будет дана характеризация колец $\mathscr{A}[\xi]^{\mathscr{H}}$ и $\mathscr{B}[\xi]^{\mathscr{H}}$. Эта характеризация будет затем применена к ряду $G(\lambda) \in \mathscr{B}[\ell]$ из теоремы 4.1. 
Итак, пусть $\mathscr{H}$ - некоторая подгруппа группы $G$ порядка $\ell^{s}$, где $1 \leqslant s \leqslant k$. Для произвольного натурального $m$ положим

$$
T_{\langle m\rangle}=T_{\langle m\rangle}(T)=1-(1-T)^{\ell^{m}}=\sum_{i=1}^{\ell^{m}}\left(\begin{array}{c}
\ell^{m} \\
i
\end{array}\right)(-1)^{i+1} T^{i} .
$$

Для любого элемента $\sigma \in \mathscr{H}$ мы имеем $\sigma(1-T)=\xi_{\sigma}(1-T)$, поэтому $\sigma\left(T_{\langle s\rangle}\right)=T_{\langle s\rangle}$, т.e. $T_{\langle s\rangle} \in \mathscr{A}[\xi]^{\mathscr{H}}$.

Пусть $C(X) \in \mathscr{O}_{H}[\xi]\{\{X\}\}$. Поскольку $T_{\langle s\rangle} \equiv \pm T^{\ell^{s}}(\bmod \ell)$ и $T_{\langle s\rangle}^{-1}= \pm T^{-\ell^{s}} \times$ $U(T)$, где $U(T) \in \mathscr{A}[\ell]^{\times}$и $U(T) \equiv 1(\bmod \ell)$, мы получаем, что ряд $i\left(C\left(T_{\langle s\rangle}\right)\right):=$ $C\left(T_{\langle s\rangle}(T)\right)$ является корректно определенным элементом кольца $\mathscr{A}[\xi]$, что определяет гомоморфизм колец

$$
i: \mathscr{O}_{H}[\xi]\left\{\left\{T_{\langle s\rangle}\right\}\right\} \longrightarrow \mathscr{A}[\xi]
$$

который, как легко можно проверить, является вложением. Пользуясь этим вложением, мы будем рассматривать $\mathscr{O}_{H}[\xi]\left\{\left\{T_{\langle s\rangle}\right\}\right\}$ как подкольцо кольца $\mathscr{A}[\xi]$.

ПРЕДЛОЖЕНИЕ 5.1. Пусть $1 \leqslant s \leqslant k u \mathscr{H}-$ nодгруппа группы $G$ порядка $\ell^{s}$. Тогда

$$
\mathscr{A}[\xi]^{\mathscr{H}}=\mathscr{O}_{H}[\xi]\left\{\left\{T_{\langle s\rangle}\right\}\right\} .
$$

Другими словами, любой ряд Лорана от $T$ из кольца $\mathscr{A}[\xi]$, неподвижный относительно Н , может быть представлен как ряд Лорана от $T_{\langle s\rangle}$.

ДокАЗАТЕльСтво. Обозначим кольцо $\mathscr{O}_{H}[\xi]\left\{\left\{T_{\langle s\rangle}\right\}\right\}$ через $\mathbb{A}[\xi]$. Мыпокажем, что $\mathscr{A}[\xi]$ порождается элементами $1, T, \ldots, T^{\ell^{s}-1}$ как $\mathbb{A}[\xi]$-модуль. Действительно, поскольку $T_{\langle s\rangle} \equiv \pm T^{\ell^{s}}(\bmod \ell)$, мы получаем, что любой ряд $f(T) \in \mathscr{A}[\xi]$ представим в виде

$$
f(T) \equiv \sum_{i=0}^{\ell^{s}-1} f_{i, 1}\left(T_{\langle s\rangle}\right) T^{i} \quad(\bmod \pi)
$$

где $f_{i, 1}\left(T_{\langle s\rangle}\right) \in \mathscr{O}_{H}[\xi]\left[\left[T_{\langle s\rangle}\right]\right]$, коэффициенты рядов $f_{i, 1}$ определены однозначно по модулю $\pi$ и $\pi$ - локальньй параметр кольца $\mathscr{O}_{H}[\xi]$. Предположим, что для некоторого $n \geqslant 1$ уже построены формальные ряды Лорана с конечной главной частью:

$$
f_{i, n}\left(T_{\langle s\rangle}\right) \in \mathscr{O}_{H}[\xi]\left(\left(T_{\langle s\rangle}\right)\right), \quad 0 \leqslant i \leqslant \ell^{s}-1,
$$

такие, что все коэффициенты при отрицательных степенях $T_{\langle s\rangle}$ делятся на $\pi$, и вьполняется сравнение

$$
f(T) \equiv \sum_{i=0}^{\ell^{s}-1} f_{i, n}\left(T_{\langle s\rangle}\right) T^{i} \quad\left(\bmod \pi^{n}\right) .
$$

Тогда, как и в (5.1), мы можем найти формальные ряды Лорана с конечной главной частью $g_{i}\left(T_{\langle s\rangle}\right), 0 \leqslant i \leqslant \ell^{s}-1$, такие, что

$$
f(T)-\sum_{i=0}^{\ell^{s}-1} f_{i, n}\left(T_{\langle s\rangle}\right) T^{i} \equiv \pi^{n} \sum_{i=0}^{\ell^{s}-1} g_{i}\left(T_{\langle s\rangle}\right) T^{i} \quad\left(\bmod \pi^{n+1}\right) .
$$


Полагая $f_{i, n+1}\left(T_{\langle s\rangle}\right)=f_{i, n}\left(T_{\langle s\rangle}\right)+\pi^{n} g_{i}\left(T_{\langle s\rangle}\right)$, мы получаем, что сравнение вида (5.2) выполняется и для $n+1$. Полагая $f_{i}\left(T_{\langle s\rangle}\right)=\lim _{n \rightarrow \infty} f_{i, n}\left(T_{\langle s\rangle}\right)$ для $0 \leqslant$ $i \leqslant \ell^{s}-1$ и учитьвая, что $f_{i, n}\left(T_{\langle s\rangle}\right)$ имеет конечную главную часть при любом $n$, мы получаем, что $f_{i}\left(T_{\langle s\rangle}\right) \in \mathbb{A}[\xi]$ для $0 \leqslant i \leqslant \ell^{s}-1$ и $f(T)=\sum_{i=0}^{\ell^{s}-1} f_{i}\left(T_{\langle s\rangle}\right) T^{i}$. Это означает, что $1, T, \ldots, T^{\ell^{s}-1}$ порождают $\mathscr{A}[\xi]$ как $\mathbb{A}[\xi]$-модуль.

Пусть $\mathscr{K}$ и $\mathscr{L}$ - поля частных колец $\mathbb{A}[\xi]$ и $\mathscr{A}[\xi]$ соответственно. Тогда элементы $1, T, \ldots, T^{\ell^{s}-1}$ порождают $\mathscr{L}$ над $\mathscr{K}$. Группа $\mathscr{H}$ действует на $\mathscr{L}$, и $\mathscr{L} / \mathscr{L}^{\mathscr{H}}$ является расширением Галуа степени $\ell^{s}$, причем $\mathscr{L} \supset \mathscr{L}^{\mathscr{H}} \supseteq \mathscr{K}$. Следовательно, $\mathscr{L}^{\mathscr{H}}=\mathscr{K}$.

Поскольку $T$ удовлетворяет уравнению $1-(1-T)^{\ell^{s}}-T_{\langle s\rangle}=0$, мы получаем, что $\mathscr{A}[\xi]$ цело над $\mathbb{A}[\xi]$. Тогда из условия $x \in \mathscr{A}[\xi]^{\mathscr{H}}$ следует, что $x \in \mathscr{A}[\xi] \cap \mathscr{K}$, откуда следует, что $x \in \mathscr{K}$ и $x$ цел над $\mathbb{A}[\xi]$, но согласно предложению 3.2 кольцо $\mathbb{A}[\xi]$ целозамкнуто в $\mathscr{K}$, откуда вытекает, что $x \in \mathbb{A}[\xi]$. Предложение доказано.

Теперь мы дадим характеризацию кольца $\mathscr{B}[\xi]^{\mathscr{H}}$. Мы рассмотрим сначала случай, когда $|\mathscr{H}|=\ell$, а затем покажем, что общий случай легко сводится к этому частному случаю.

ТЕОРема 5.1. Пусть $F(X, T)$ - многочлен из предложения $1.3 \quad$ и $F^{(1)}(X, T)$ - многочлен, полученный из $F(X, T)$ действием автоморфизма Фробениуса $\varphi$ на коэффициенты. Пусть $T_{\langle 1\rangle}(\lambda)$ - результат композиции рядов $T_{\langle 1\rangle}(T)$ и $T(\lambda)$. Тогда в кольце $\mathscr{B}[\ell]$ существует единственный элемент $\lambda_{\langle 1\rangle}=\lambda_{\langle 1\rangle}(\lambda)=Q(\lambda)=\sum_{j=-\infty}^{\infty} q_{j} \lambda^{j} \quad$ maкой, чmo $\lambda_{\langle 1\rangle}(\lambda) \equiv \lambda^{\ell}(\bmod \ell) \quad u$ $F^{(1)}\left(\lambda_{\langle 1\rangle}(\lambda), T_{\langle 1\rangle}(\lambda)\right)=0$. При әтом для всех $j<0$ виполняется неравенство

$$
\nu_{\ell}\left(q_{j}\right)>-\frac{j}{2 \delta \ell}
$$

где $\delta$ имеет тот же смысл, что и в предложении 1.2 .

ЗАмЕчАниЕ. В дальнейшем мы будем часто иметь дело с условиями вида (5.3), поэтому нам удобно ввести следуюшее определение. Для любого кольца формальных рядов Лорана $\mathscr{O}_{H}\{\{X\}\}$ вида (3.1) и произвольного натурального $r$ положим

$$
\begin{gathered}
\mathscr{O}_{H}\{\{X\}\}_{r}=\left\{A(X) \in \mathscr{O}_{H}\{\{X\}\}, \quad A(X)=\sum_{j=-\infty}^{\infty} a_{j} X^{j} \mid\right. \\
\left.\nu_{\ell}\left(a_{j}\right)>-\frac{j}{2 \delta \ell^{r}} \text { при всех } j<0\right\} .
\end{gathered}
$$

Через $\mathscr{O}_{H}\{\{X\}\}_{r}^{(0)}$ обозначим множество всех тех рядов из $\mathscr{O}_{H}\{\{X\}\}_{r}$, все коэффициенты которых делятся на $\ell$. Без труда проверяется, что $\mathscr{O}_{H}\{\{X\}\}_{r}$ является подкольцом в $\mathscr{O}_{H}\{\{X\}\}$, а $\mathscr{O}_{H}\{\{X\}\}_{r}^{(0)}$ является идеалом в $\mathscr{O}_{H}\{\{X\}\}_{r}$.

На языке этого определения условие (5.3) означает, что $\lambda_{\langle 1\rangle}(\lambda) \in \mathscr{B}[\ell]_{1}$, а теорема 4.1 утверждает, что $G(\lambda) \in \mathscr{B}[\ell]_{n-p}^{(0)}$.

ДОКАЗАТЕЛЬСТВо ТЕОРЕМЫ. Поскольку

$$
F(X, T)^{\ell} \equiv F^{(1)}\left(X^{\ell}, T^{\ell}\right) \equiv F^{(1)}\left(X^{\ell}, T_{\langle 1\rangle}\right) \quad(\bmod \ell),
$$


мы получаем $F^{(1)}\left(\lambda^{\ell}, T_{\langle 1\rangle}(\lambda)\right) \equiv 0(\bmod \ell)$, т.е. $\lambda^{\ell}$ является приближенным (по модулю $\ell$ ) решением уравнения $F^{(1)}\left(X, T_{\langle 1\rangle}(\lambda)\right)=0$. Мы поднимем $\lambda^{\ell}$ до решения этого уравнения тем же способом, что и в доказательстве теоремы 3.1 .

Пусть $H^{(1)}(X, T)$ - формальная производная многочлена $F^{(1)}(X, T)$ по $X$. Поскольку $T_{\langle 1\rangle} \equiv T^{\ell}(\bmod \ell)$, мы получаем, используя определение $\delta$, что

$$
H^{(1)}\left(\lambda^{\ell}, T_{\langle 1\rangle}(\lambda)\right) \equiv \lambda^{\ell \delta} h(\lambda) \quad(\bmod \ell)
$$

где $h(\lambda)$ - некоторый обратимый формальный степенной ряд.

Мы определим последовательность приближений $Q_{i}(\lambda)$, где $Q_{i}(\lambda) \in \mathscr{O}_{H}((\lambda))$, т.е. $Q_{i}(\lambda)$ - ряд Лорана с конечной главной частью, таких, что

$$
\begin{gathered}
F^{(1)}\left(Q_{i}(\lambda), T_{\langle 1\rangle}(\lambda)\right) \equiv 0\left(\bmod \ell^{i}\right) \\
Q_{i+1}(\lambda) \equiv Q_{i}(\lambda)\left(\bmod \ell^{i}\right)
\end{gathered}
$$

для всех $i \geqslant 0$.

Положим $Q_{1}(\lambda)=\lambda^{\ell}$. Если элемент $Q_{i}(\lambda)$ уже определен, то мы положим

$$
Q_{i+1}(\lambda) \equiv Q_{i}(\lambda)-\frac{F^{(1)}\left(Q_{i}(\lambda), T_{\langle 1\rangle}(\lambda)\right)}{\lambda^{\ell \delta} h(\lambda)} \quad\left(\bmod \ell^{i+1}\right) .
$$

Условие (5.6) определяет коэффициенты ряда $Q_{i+1}(\lambda)$ только по модулю $\ell^{i+1}$. Поэтому мы определим $Q_{i+1}(\lambda)$ как некоторый ряд, удовлетворяюший сравнению (5.6) и такой, что среди его ненулевых коэффициентов нет коэффициентов, делящихся на $\ell^{i+1}$. Применяя к нашему уравнению аналог формулы (3.14) и учитывая, что согласно (5.5)

$$
H^{(1)}\left(Q_{i}(\lambda), T_{\langle 1\rangle}(\lambda)\right) \equiv \lambda^{\ell \delta} h(\lambda) \quad(\bmod \ell)
$$

при любом $i \geqslant 1$, мы получаем, что из справедливости (5.4) для некоторого $i$ следует справедливость для этого же $i$ сравнения (5.5), а также справедливость сравнения (5.4) для $i+1$. Следовательно, полагая $Q(\lambda)=\lim _{i \rightarrow \infty} Q_{i}(\lambda)$ (в смысле покоэффициентной сходимости), мы получаем

$$
\begin{aligned}
& F^{(1)}\left(Q(\lambda), T_{\langle 1\rangle}(\lambda)\right)=0 \\
& Q(\lambda) \equiv Q_{i}(\lambda)\left(\bmod \ell^{i}\right)
\end{aligned}
$$

для любого $i \geqslant 1$.

Нам осталось проверить только, что для $Q(\lambda)$ выполняется неравенство (5.3), т.е. что $Q(\lambda) \in \mathscr{B}[\ell]_{1}$. Как и в доказательстве теоремы 3.1 , мы будем доказывать индукцией по $i$, что для всех коэффициентов $q_{i, j}$ ряда $Q_{i}(\lambda)$ выполняется более сильное неравенство:

3 Серия математическая, №6 $\nu_{\ell}\left(q_{i, j}\right)>-\frac{1}{2}\left[\frac{j}{\delta \ell}\right]$ при всех $j<0$. 
Предположим, что (5.9) уже доказано для коэффициентов ряда $Q_{i}(\lambda)$ при некотором $i \geqslant 1$. Тогда в силу (5.6) нам достаточно доказать, что аналог (5.9) выполняется для всех тех коэффициентов $r_{i, j}$ ряда

$$
\sum_{j=-\infty}^{\infty} r_{i, j} \lambda^{j}:=\frac{F^{(1)}\left(Q_{i}(\lambda), T_{\langle 1\rangle}(\lambda)\right)}{\lambda^{\ell \delta} h(\lambda)}
$$

для которых $\nu_{\ell}\left(r_{i, j}\right)<i+1$. Поскольку для $Q_{i}(\lambda)$ выполняется сравнение $(5.4)$, мы имеем

$$
r_{i, j} \equiv 0 \quad\left(\bmod \ell^{i}\right) \quad \text { для всех } j .
$$

Таким образом, нам достаточно проверить, что для всех тех коэффициентов $r_{i, j}$ ряда (5.10), для которых $\nu_{\ell}\left(r_{i, j}\right)=i$, выполняется неравенство

$$
i>-\frac{1}{2}\left[\frac{j}{\delta \ell}\right]
$$

Как и в доказательстве теоремы 3.1, ряд (5.10) можно представить в виде суммы одночленов вида

$$
a_{t} \lambda^{t} \times \lambda^{-\ell \delta} h_{s}^{\prime} \lambda^{s} \times \prod_{\alpha=1}^{r} q_{i, j_{\alpha}} \lambda^{j_{\alpha}}
$$

где $a_{t} \lambda^{t}-$ один из одночленов, входящих в коэффициент многочлена $F^{(1)}\left(X, T_{\langle 1\rangle}(\lambda)\right)$ при $X^{r}$ для $r \geqslant 0$, причем при $r=0$ произведение по $\alpha=1, \ldots, r$ в (5.12) предполагается равным 1 , а $h_{s}^{\prime}$ - коэффициент при $\lambda^{s}$ ряда $h(\lambda)^{-1}$. Таким образом, одночлен (5.12) имеет степень

$$
j=s+t-\ell \delta+\sum_{\alpha=1}^{r} j_{\alpha}
$$

где при $r=0$ сумма от 1 до $r$ предполагается равной нулю.

Если $r=0$, то $j \geqslant-\ell \delta$. Поскольку $\nu_{\ell}\left(r_{i, j}\right) \geqslant i \geqslant 1$, мы получаем, что член $r_{i, j} \lambda^{j}$, который является суммой всех одночленов вида (5.12) с заданным значением $j$, удовлетворяет неравенству (5.11).

Пусть $r \geqslant 1$ и лишь один из показателей $j_{1}, \ldots, i_{r}$ отрицателен; например, пусть $j_{1}<0$ и $j_{2}, \ldots, j_{r} \geqslant 0$. Тогда согласно (5.13) $\lambda$ входит в (5.12) с показателем $j \geqslant j_{1}-\ell \delta$. Поскольку и в этом случае одночлен (5.12) входит в член $r_{i, j} \lambda^{j}$, где $\nu_{\ell}\left(r_{i, j}\right) \geqslant i$, учитывая (5.9) и то, что

$$
\left[\frac{j}{\ell \delta}\right] \geqslant\left[\frac{j_{1}-\ell \delta}{\ell \delta}\right]=\left[\frac{j_{1}}{\ell \delta}\right]-1
$$

мы получаем

$$
i \geqslant \nu_{\ell}\left(q_{i, j_{1}}\right)+1>-\frac{1}{2}\left[\frac{j_{1}}{\ell \delta}\right]+1>-\frac{1}{2}\left(\left[\frac{j_{1}}{\ell \delta}\right]-1\right) \geqslant-\frac{1}{2}\left[\frac{j}{\ell \delta}\right] .
$$


Таким образом, и в этом случае одночлен (5.12) входит в член $r_{i, j} \lambda^{j}$, удовлетворяюший условию (5.11).

Наконец, предположим, что в (5.12) имеются хотя бы два отрицательных показателя. Например, пусть $j_{1}, j_{2}<0$, а остальные $j_{\alpha}$ произвольны. Тогда, рассуждая так же, как и в доказательстве теоремы 3.1 , мы получаем

$$
\nu_{\ell}\left(q_{i, j_{1}} q_{i, j_{2}}\right)>\frac{1}{2}\left[\frac{j_{1}+j_{2}-\ell \delta}{\ell \delta}\right]
$$

откуда, снова рассуждая, как и в доказательстве теоремы 3.1 , мы получаем, что в этом случае уже для самого одночлена (5.12) справедливо неравенство вида (5.11), т.е. что

$$
\nu_{\ell}\left(a_{t} h_{s}^{\prime} \prod_{\alpha=1}^{r} q_{i, j_{\alpha}}\right)>\frac{1}{2}\left[\frac{j}{\ell \delta}\right]
$$

где $j$ определяется формулой (5.13). Этим теорема доказана полностью.

ПредлОЖенИЕ 5.2. Пусть $\mathscr{H}$ - подгруппа порядка $\ell$ группь $G$. Тогда $\lambda_{\langle 1\rangle} \in \mathscr{B}[\xi]^{\mathscr{H}}$.

ДоКАЗАТЕЛЬСТво. Учитывая, что $\sigma\left(T_{\langle 1\rangle}\right)=T_{\langle 1\rangle}$ для любого $\sigma \in \mathscr{H}$, мы получаем

$$
0=\sigma\left(F^{(1)}\left(\lambda_{\langle 1\rangle}, T_{\langle 1\rangle}(\lambda)\right)\right)=F^{(1)}\left(\sigma\left(\lambda_{\langle 1\rangle}\right), T_{\langle 1\rangle}(\lambda)\right) .
$$

Согласно теореме $3.2 \sigma(\lambda) \equiv \lambda\left(\bmod 1-\xi_{\sigma}\right)$, поэтому для элемента $\sigma$ порядка $\ell$ мы имеем $\sigma\left(\lambda^{\ell}\right) \equiv \lambda^{\ell}(\bmod \ell)$. Поскольку и $\lambda_{\langle 1\rangle} \equiv \lambda^{\ell}(\bmod \ell)$, из теоремы 5.1 следует, что $\sigma\left(\lambda_{\langle 1\rangle}\right)=\lambda_{\langle 1\rangle}$. Предложение доказано.

Положим $\lambda_{\langle 2\rangle}=Q^{(1)}(Q(\lambda))$, где $Q(\lambda)=\lambda_{\langle 1\rangle}$ - ряд из теоремы 5.1. Вообше, если ряд $\lambda_{\langle m\rangle}=\lambda_{\langle m\rangle}(\lambda)$ уже определен, то мы полагаем $\lambda_{\langle m+1\rangle}=Q^{(m)}\left(\lambda_{\langle m\rangle}(\lambda)\right)$, где $Q^{(m)}(X)$ - ряд, полученный действием $\varphi^{m}$ на коэффициенты ряда $Q(X)$.

Последовательно применяя (5.7) $m$ раз, мы получаем

$$
F^{(m)}\left(\lambda_{\langle m\rangle}(\lambda), T_{\langle m\rangle}(\lambda)\right)=0,
$$

т.е. $\lambda_{\langle m\rangle}$ - корень уравнения $F^{(m)}\left(X, T_{\langle m\rangle}(\lambda)\right)=0$, при этом $\lambda_{\langle m\rangle}$ однозначно выделяется среди других корней этого уравнения условием $\lambda_{\langle m\rangle} \equiv \lambda^{\ell^{m}}(\bmod \ell)$.

ПреДЛОЖЕНИЕ 5.3. Пусть $\mathscr{H}$ - nодгруппа порядка $\ell^{s}$ группь G. Тогда подстановка $\lambda_{\langle s\rangle}=\lambda_{\langle s\rangle}(\lambda)$ определяет естественное вложение

$$
i: \mathscr{O}_{H}[\xi]\left\{\left\{\lambda_{\langle s\rangle}\right\}\right\} \hookrightarrow \mathscr{B}[\xi],
$$

которое позволяет рассматривать $\mathscr{O}_{H}[\xi]\left\{\left\{\lambda_{\langle s\rangle}\right\}\right\}$ как подкольчо кольца $\mathscr{B}[\xi]$. При этом Н действует тождественно на $\lambda_{\langle s\rangle}$,

$$
\begin{gathered}
\mathscr{B}[\xi]^{\mathscr{H}}=\mathscr{O}_{H}[\xi]\left\{\left\{\lambda_{\langle s\rangle}\right\}\right\}, \\
\mathscr{O}_{H}\left\{\left\{\lambda_{\langle s\rangle}\right\}\right\}=\mathscr{B}[\ell] \cap \mathscr{O}_{H}[\xi]\left\{\left\{\lambda_{\langle s\rangle}\right\}\right\} .
\end{gathered}
$$


ДокАЗАТЕЛЬСТВО. Поскольку $\lambda_{\langle s\rangle} \equiv \lambda^{\ell^{s}}(\bmod \ell)$, ряд $f\left(\lambda_{\langle s\rangle}(\lambda)\right)$ является корректно определенным элементом кольца $\mathscr{B}[\xi]$ для любого $f(X) \in \mathscr{O}_{H}\{\{X\}\}$. Инъективность $i$ вытекает из сравнения $\lambda_{\langle s\rangle} \equiv \lambda^{\ell^{s}}(\bmod \ell)$.

То, что $\mathscr{H}$ действует тождественно на $\lambda_{\langle s\rangle}$, можно доказать индукцией по $s$. Действительно, для $s=1$ наше утверждение доказано в предложении 5.2.

Пусть $\mathscr{H}_{1}$ - подгруппа индекса $\ell$ групшы $\mathscr{H}$. По предположению индукции мы можем считать, что $\mathscr{H}_{1}$ действует тождественно на $T_{\langle s-1\rangle}$ и на $\lambda_{\langle s-1\rangle}$. Тогда на $\mathscr{O}_{H}[\xi]\left\{\left\{\lambda_{\langle s-1\rangle}\right\}\right\}$ действует группа $\mathscr{H} / \mathscr{H}_{1}$ порядка $\ell$, и, применяя в этой ситуации предложение 5.2 , мы получаем, что $\mathscr{H}$ действует тождественно на $\lambda_{\langle s\rangle}$, т.е. $\mathscr{O}_{H}[\xi]\left\{\left\{\lambda_{\langle s\rangle}\right\}\right\} \subseteq \mathscr{B}[\xi]^{\mathscr{H}}$. Рассуждая, как и в доказательстве предложения 5.1, мы получаем, что $\mathscr{B}[\xi]$ порождается как $\mathscr{O}_{H}[\xi]\{\{\lambda\langle s\rangle\}\}$-модуль элементами $1, \lambda, \ldots, \lambda^{\ell^{s}-1}$, откуда, как и в доказательстве предложения 5.1 , мы получаем

$$
\mathscr{B}[\xi]^{\mathscr{H}}=\mathscr{O}_{H}[\xi]\left\{\left\{\lambda_{\langle s\rangle}\right\}\right\} .
$$

Если $F(\lambda) \in \mathscr{B}[\ell] \cap \mathscr{B}[\xi]^{\mathscr{H}}$, то согласно уже доказанному $F(\lambda)=F_{1}\left(\lambda_{\langle s\rangle}\right)$ для некоторого $F_{1}(X) \in \mathscr{O}_{H}[\xi]\{\{X\}\}$. Если не все коэффициенты $F_{1}(X)$ принадлежат $\mathscr{O}_{H}$, то пусть $\ell^{n}$ - максимальная степень $\ell$, для которой существует ряд $A(X) \in \mathscr{O}_{H}\{\{X\}\}$ такой, что $F_{1}(X) \equiv A(X)\left(\bmod \ell^{n}\right)$. Тогда ряд $B\left(\lambda_{\langle s\rangle}\right):=$ $F_{1}\left(\lambda_{\langle s\rangle}\right)-A\left(\lambda_{\langle s\rangle}\right)$ обладает тем свойством, что $B\left(\lambda_{\langle s\rangle}\right) \equiv 0\left(\bmod \ell^{n}\right)$, и сушествует коэффициент $b_{i}$ ряда $B(X)$ такой, что $b_{i} \bmod \ell^{n+1} \notin \mathscr{O}_{H} / \ell^{n+1} \mathscr{O}_{H}$. Очевидно, что $B\left(\lambda_{\langle s\rangle}(\lambda)\right)=F(\lambda)-A\left(\lambda_{\langle s\rangle}(\lambda)\right) \in \mathscr{O}_{H}\{\{\lambda\}\}$, что противоречит сравнению

$$
B\left(\lambda_{\langle s\rangle}(\lambda)\right) \equiv \sum_{j=-\infty}^{\infty} b_{j} \lambda^{\ell^{s} j} \quad\left(\bmod \ell^{n+1}\right)
$$

Предложение доказано.

Tеорема 5.2. Пусть $G(\lambda)=G\left(n-p-1, \varphi^{n}(\alpha), a, \lambda\right)-$ формальный ряд Лорана из теоремы 4.1. Тогда существует формальный ряд Лорана $D(X)=$ $\sum_{i=-\infty}^{\infty} d_{i} X^{i}$ такой, что $G(\lambda)=D\left(\lambda_{\langle s\rangle}(\lambda)\right)$, где $s=n-p-1$. При этом $D(X) \in \mathscr{O}_{H}\{\{X\}\}_{1}^{(0)}$, m.e. $d_{i} \in \ell \mathscr{O}_{H} \partial л$ s всех $i \in \mathbb{Z} u$

$$
\nu_{\ell}\left(d_{i}\right)>-\frac{i}{2 \delta \ell}
$$

для всех $i<0$.

ДокАЗАТЕЛЬСТво. Пусть $\mathscr{H}$ - подгруппа индекса $\ell$ в $G$. Напомним, что $G$ имееет порядок $\ell^{n-p}$. Соответственно порядок $\mathscr{H}$ равен $\ell^{s}$.

Из определения рядов $G_{r}(\lambda)$ в $(4.7)$ и теоремы 3.2 следует, что ряды $G_{r}(\lambda)$ остаются неподвижными относительно действия $\mathscr{H}$. Следовательно, и $G(\lambda)$ неподвижен относительно действия $\mathscr{H}$. Тогда согласно предложению $5.4 G(\lambda)=$ $D\left(\lambda_{\langle s\rangle}(\lambda)\right)$ для некоторого ряда $D(X) \in \mathscr{O}_{H}\{\{X\}\}$.

Нам осталось проверить, что $D(X) \in \mathscr{O}_{H}\{\{X\}\}_{1}^{(0)}$. Для этого мы заметим, что для любого индекса $r, 1 \leqslant r \leqslant s$, ряд $G(\lambda)$ неподвижен относительно действия единственной подгруппы группы $\mathscr{H}$ порядка $\ell^{r}$. Поэтому в силу предложения 5.4 
для любого такого $r$ сушествует формальный ряд $D_{r}(X) \in \mathscr{O}_{H}\{\{X\}\}$ такой, что $G(\lambda)=D_{r}\left(\lambda_{\langle r\rangle}(\lambda)\right)$. При этом $D_{r}(X)=D_{r+1}\left(\lambda_{\langle 1\rangle}(X)\right)$ для $r=1, \ldots, s-1$. Отметим, что $D_{s}(X)=D(X)$.

Прежде всего заметим, что из сравнений $G(\lambda) \equiv 0(\bmod \ell)$ и $\lambda_{\langle r\rangle} \equiv \lambda^{\ell^{r}}(\bmod \ell)$ мы получаем

$$
0 \equiv G(\lambda) \equiv D_{r}\left(\lambda^{\ell^{r}}\right) \quad(\bmod \ell),
$$

откуда следует, что все коэффициенты ряда $D_{r}(X)$ делятся на $\ell$ для любого $1 \leqslant r \leqslant s$.

Чтобы доказать (5.16), мы докажем, что для любых двух рядов $F(X), H(X) \in$ $\mathscr{O}_{H}\{\{X\}\}$ таких, что $H(X)=F\left(\lambda_{\langle 1\rangle}(X)\right)$, условие $F(X) \in \mathscr{O}_{H}\{\{X\}\}_{m}$ равносильно условию $H(X) \in \mathscr{O}_{H}\{\{X\}\}_{m+1}$.

Лемma 5.1. Пусть $F(X), H(X) \in \mathscr{O}_{H}\{\{X\}\}, H(X)=F\left(\lambda_{\langle 1\rangle}(X)\right)$ u $F(X) \in$ $\mathscr{O}_{H}\{\{X\}\}_{m}$. Tогдa $H(X) \in \mathscr{O}_{H}\{\{X\}\}_{m+1}$.

ДоКАЗАТЕЛЬСТво. Ряд $F(X)$ является суммой одночленов $F_{i}(X)=f_{i} X^{i}$, $-\infty<i<\infty$, и нам достаточно проверить, что для любого целого $i$ ряд $H_{i}(X):=$ $f_{i} \lambda_{\langle 1\rangle}(X)^{i}$ принадлежит $\mathscr{O}_{H}\{\{X\}\}_{m+1}$.

Если $i>0$, то, учитывая, что $\lambda_{\langle 1\rangle}(X) \in \mathscr{O}_{H}\{\{X\}\}_{1}$, мы получаем $H_{i}(X) \in$ $\mathscr{O}_{H}\{\{X\}\}_{1} \subseteq \mathscr{O}_{H}\{\{X\}\}_{m+1}$.

Если $i=0$, то $H_{0}(X)=f_{0} \in \mathscr{O}_{H} \subset \mathscr{O}_{H}\{\{X\}\}_{m+1}$.

Предположим теперь, что $i<0$. Из теоремы 5.1 следует, что

$$
\lambda_{\langle 1\rangle}(X)=q_{\ell} X^{\ell}\left(1+\sum_{\substack{-\infty<j<\infty \\ j \neq \ell}} q_{\ell}^{-1} q_{j} X^{j-\ell}\right),
$$

где $q_{\ell} \equiv 1(\bmod \ell)$ и $q_{\ell}^{-1} q_{j} \equiv 0(\bmod \ell)$ для всех $j \neq \ell$. Положим

$$
M(X)=\sum_{\nu=-\infty}^{\infty} m_{\nu} X^{\nu}=\sum_{\substack{-\infty j<\infty \\ j \neq \ell}} q_{\ell}^{-1} q_{j} X^{j-\ell} .
$$

Тогда

$$
\lambda_{\langle 1\rangle}(X)^{-1}=q_{\ell}^{-1} X^{-\ell}\left(1+\sum_{n=1}^{\infty}(-1)^{n} M(X)^{n}\right) .
$$

Мы покажем, что $M(X) \in \mathscr{O}_{H}\{\{X\}\}_{2}$, т.е. что для всех $\nu<0$ справедливо неравенство

$$
\nu_{\ell}\left(m_{\nu}\right)>-\frac{\nu}{2 \ell^{2} \delta}
$$

Действительно, если $\nu \leqslant-(\ell+2)$, то $-(\nu+\ell) \ell \geqslant-\nu$, откуда следует, что

$$
\nu_{\ell}\left(m_{\nu}\right)=\nu_{\ell}\left(q_{\nu+\ell}\right)>-\frac{(\nu+\ell) \ell}{2 \ell^{2} \delta} \geqslant-\frac{\nu}{2 \ell^{2} \delta} .
$$


Если $\nu>-(\ell+2)$, то $\nu_{\ell}\left(m_{\nu}\right) \geqslant 1>-\nu /\left(2 \ell^{2} \delta\right)$. Следовательно, $M(X) \in$ $\mathscr{O}_{H}\{\{X\}\}_{2}$. Тогда и $M(X)^{n} \in \mathscr{O}_{H}\{\{X\}\}_{2}$ для любого $n$, откуда следует, что

$$
1+N(X):=1+\sum_{n=1}^{\infty}(-1)^{n} M(X)^{n} \in \mathscr{O}_{H}\{\{X\}\}_{2}
$$

Итак, пусть $H_{i}(X)=f_{i} \lambda_{\langle 1\rangle}(X)^{i}$ для $i<0$. Тогда

$$
H_{i}(X)=f_{i} q_{\ell}^{i} X^{i \ell}(1+N(X))^{-i}
$$

Согласно условию леммы $F(X) \in \mathscr{O}_{H}\{\{X\}\}_{m}$, т.е. $\nu_{\ell}\left(f_{i}\right)>-i /\left(2 \ell^{m} \delta\right)$, откуда следует, что

$$
f_{i} q_{\ell}^{i} X^{i \ell} \in \mathscr{O}_{H}\{\{X\}\}_{m+1} .
$$

Согласно (5.18) множитель $(1+N(X))^{-i}$ в (5.19) также принадлежит $\mathscr{O}_{H}\{\{X\}\}_{2}$ (напомним, что $-i-$ натуральное число). Поскольку $m+1 \geqslant 2$, мы имеем $\mathscr{O}_{H}\{\{X\}\}_{2} \subseteq \mathscr{O}_{H}\{\{X\}\}_{m+1}$. Лемма доказана.

Лемма 5.2. Пусть, как и в лемме 5.1, $F(X), H(X) \in \mathscr{O}_{H}\{\{X\}\}$ и $H(X)=$ $F\left(\lambda_{\langle 1\rangle}(X)\right)$. Пусть $H(X) \in \mathscr{O}_{H}\{\{X\}\}_{m+1}$ для некоторого $m \geqslant 1$. Тогда $F(X) \in \mathscr{O}_{H}\{\{X\}\}_{m}$.

ДокАЗАТЕЛЬСТво. Предположим, что $F(X) \notin \mathscr{O}_{H}\{\{X\}\}_{m}$. Это означает, что существует индекс $j_{0}<0$ такой, что

$$
\nu_{\ell}\left(f_{j_{0}}\right) \leqslant-\frac{j_{0}}{2 \ell^{m} \delta}, \quad \text { где } F(X)=\sum_{j=-\infty}^{\infty} f_{j} X^{j}
$$

Не ограничивая общности, мы можем считать, что $j_{0}$ - максимальный индекс, для которого выполняется (5.21). Рассмотрим представление $F(X)=F_{1}(X)+$ $F_{2}(X)$, где $F_{1}(X)=\sum_{j=-\infty}^{j_{0}} f_{j} X^{j}$ и $F_{2}(X)=\sum_{j=j_{0}+1}^{\infty} f_{j} X^{j}$. Из определения индекса $j_{0}$ следует, что $F_{2}(X) \in \mathscr{O}_{H}\{\{X\}\}_{m}$. Тогда в силу леммы 5.2 мы имеем $H_{2}(X):=F_{2}\left(\lambda_{\langle 1\rangle}(x)\right) \in \mathscr{O}_{H}\{\{X\}\}_{m+1}$. Положим $H_{1}(X)=H(X)-H_{2}(X)$. Тогда из условия $H(X), H_{2}(X) \in \mathscr{O}_{H}\{\{X\}\}_{m+1}$ следует, что и

$$
H_{1}(X) \in \mathscr{O}_{H}\{\{X\}\}_{m+1} .
$$

С другой стороны, $H_{1}(X)=F_{1}\left(\lambda_{\langle 1\rangle}(X)\right)$. Пусть $\ell^{t}$ - максимальная степень $\ell$ такая, что $F_{1}(X) \equiv 0\left(\bmod \ell^{t-1}\right)$, и пусть $j_{1}-$ максимальный индекс такой, что $f_{j_{1}} \not \equiv 0\left(\bmod \ell^{t}\right)$. Тогда $j_{1} \leqslant j_{0}$ и $\nu_{\ell}\left(f_{j_{1}}\right) \leqslant \nu_{\ell}\left(f_{j_{0}}\right)$, откуда следует, что

$$
\nu_{\ell}\left(f_{j_{1}}\right) \leqslant-\frac{j_{1}}{2 \ell^{m} \delta} .
$$

Поскольку $H_{1}(X) \equiv F_{1}\left(X^{\ell}\right)\left(\bmod \ell^{t}\right)$, мы получаем $H_{1}(X) \equiv f_{j_{1}} X^{\ell j_{1}}+\ldots$ $\left(\bmod \ell^{t}\right)$. Ввиду (5.23) это означает, что $H_{1}(X) \notin \mathscr{O}_{H}\{\{X\}\}_{m+1}$. Полученное противоречие с (5.22) доказывает лемму. 
Теперь мы легко можем закончить доказательство теоремы. Согласно теореме $4.1 G(X) \in \mathscr{O}_{H}\{\{X\}\}_{s+1}$, где $s=n-p-1$. Тогда согласно лемме 5.2 $D_{1}(X) \in \mathscr{O}_{H}\{\{X\}\}_{s}$, где $D_{1}\left(\lambda_{\langle 1\rangle}(X)\right)=G(X)$. Если для $D_{i}(X)$ уже доказано, что $D_{i}(X) \in \mathscr{O}_{H}\{\{X\}\}_{s+1-i}$, то из леммы 5.2 снова следует, что для ряда $D_{i+1}(X)$, связанного с $D_{i}(X)$ соотношением $D_{i}(X)=D_{i+1}\left(\lambda_{\langle 1\rangle}(X)\right)$, выполнено включение $D_{i+1}(X) \in \mathscr{O}_{H}\{\{X\}\}_{s-i}$. Продолжая этот процесс вплоть до ряда $D(X)=D_{s}(X)$, мы получаем, что $D(X) \in \mathscr{O}_{H}\{\{X\}\}_{1}$. Поскольку делимость всех коэффициентов ряда $D(X)$ на $\ell$ была уже проверена выше, мы получаем, что $D(X) \in \mathscr{O}_{H}\{\{X\}\}_{1}^{(0)}$. Теорема доказана.

Теорема 5.3. Пусть $D(X)$ - формальный ряд Лорана из теоремы 5.2. Тогда для любого $j \geqslant n$

$$
S_{j}:=\mathscr{F}_{j}^{(s)}\left(\eta_{j}\left(\varphi^{n-j}(\alpha), a\right)\right)=G^{(-j)}\left(\lambda_{j}\right)=D^{(-j)}\left(\lambda_{j-s}\right),
$$

где $s=n-p-1$.

ДокАЗАТЕЛЬСтво. Согласно теореме 5.2 имеется равенство формальных рядов Лорана $G(\lambda)=D\left(\lambda_{\langle s\rangle}(\lambda)\right)$. Применяя $\varphi^{-j}$ к коэффициентам в обеих частях этого равенства и подставляя $\lambda=\lambda_{j}$, мы получаем $S_{j}=G^{(-j)}\left(\lambda_{j}\right)=$ $D^{(-j)}\left(\lambda_{\langle s\rangle}^{(-j)}\left(\lambda_{j}\right)\right)$. Заметим, что сходимость ряда $\lambda_{\langle s\rangle}^{(-j)}\left(\lambda_{j}\right)$ обеспечивается неравенством (5.3). Из него же легко выводится, что $\nu_{\ell}\left(\lambda_{\langle s\rangle}^{(-j)}\left(\lambda_{j}\right)\right)=\ell^{s} \nu_{\ell}\left(\lambda_{j}\right)$, что совместно с (5.16) доказывает сходимость ряда $D^{(-j)}\left(\lambda_{\langle s\rangle}^{(-j)}\right)$.

Наконец, отметим, что согласно (5.7) мы имеем $F^{(1)}\left(\lambda_{\langle 1\rangle}(\lambda), T_{\langle 1\rangle}(\lambda)\right)=0$. Применяя к этому равенству $\varphi^{-j}$ и подставляя $\lambda=\lambda_{j}$, мы получаем $F^{(1-j)}\left(\lambda_{\langle 1\rangle}^{(-j)}\left(\lambda_{j}\right)\right.$, $\left.T_{\langle 1\rangle}^{(-j)}\left(\lambda_{j}\right)\right)=0$. Поскольку $\lambda_{j}$ удовлетворяет равенству $F^{(-j)}\left(\lambda_{j}, T^{(-j)}\left(\lambda_{j}\right)\right)=0$, мы получаем, что $T^{(-j)}\left(\lambda_{j}\right)=\pi_{j}$, откуда следует, что $T_{\langle 1\rangle}^{(-j)}\left(\lambda_{j}\right)=\pi_{j-1}$. Таким образом, $\lambda_{\langle 1\rangle}^{(-j)}\left(\lambda_{j}\right)$ - это корень уравнения $F^{(1-j)}\left(X, \pi_{j-1}\right)=0$. Поскольку $\lambda_{\langle 1\rangle}^{(-j)}\left(\lambda_{j}\right) \equiv \lambda_{j}^{\ell}\left(\bmod \ell \lambda_{j}^{-\delta}\right)$, мы получаем, что $\lambda_{\langle 1\rangle}^{(-j)}\left(\lambda_{j}\right)=\lambda_{j-1}$. Аналогичным образом мы получаем, что $\lambda_{\langle s\rangle}^{(-j)}\left(\lambda_{j}\right)=\lambda_{j-s}$. Тогда

$$
S_{j}=G^{(-j)}\left(\lambda_{j}\right)=D^{(-j)}\left(\lambda_{j-s}\right) .
$$

Теорема доказана.

\section{§ 6. Действие $\gamma_{p}-1$ на формальные ряды Лорана}

Теперь мы рассмотрим еще один тип автоморфизмов колец $\mathscr{A}[\ell]$ и $\mathscr{B}[\ell]$. Пусть $\Gamma=G\left(k_{\infty} / k_{0}\right)$, где $k_{\infty}$ и $k_{0}$ имеют тот же смысл, что и в $\S 1$. Для любого $\sigma \in \Gamma$ однозначно определен элемент $\varkappa(\sigma) \in \mathbb{Z}_{\ell}^{\times}$такой, что $\sigma\left(\zeta_{n}\right)=\zeta_{n}^{\varkappa(\sigma)}$ для любого $n$. Отображение $\varkappa$ определяет изоморфизм $\varkappa: \Gamma \cong 1+\ell \mathbb{Z}_{\ell}\left(\varkappa: \Gamma \cong 1+4 \mathbb{Z}_{2}\right.$ при $\left.\ell=2\right)$.

ПРЕДЛОЖЕНИЕ 6.1. Для любого $\sigma \in$ Г существует единственный автоморфизм кольца А्A $[\ell]$, который мы будем обозначать снова через $\sigma$, такой, что $\sigma$ действует тождественно на $\mathscr{O}_{H} и$

$$
\sigma(T)=1-(1-T)^{\varkappa(\sigma)} .
$$


Указанное действие определяет действие Г как группь автоморфизмов кольua $\mathscr{A}[\ell]$.

Если $\sigma \in \Gamma_{s}$ для некоторого $s \geqslant 0$, то для любого $F(T) \in \mathscr{O}_{H}[[T]]$ в кольце $\mathscr{O}_{H}[[T]]$ выполняются сравнения

$$
\begin{array}{rlll}
\sigma(F(T)) & \equiv F(T)\left(\bmod T_{\langle s+1\rangle}\right) & n p u & \ell \neq 2, \\
\sigma(F(T)) & \equiv F(T)\left(\bmod T_{\langle s+2\rangle}\right) & n p u & \ell=2 .
\end{array}
$$

ДоКАЗАТЕЛЬСТво. Из (6.1) следует, что $\sigma(T) \equiv T\left(\bmod T^{2}\right)$ для любого $\sigma \in \Gamma$, поэтому для любого ряда $F(T) \in \mathscr{A}[\ell]$ ряд $F(\sigma(T))$ является корректно определенным элементом кольца $\mathscr{A}[\ell]$. Полагая $\sigma(F(T))=F(\sigma(T))$, мы определяем действие $\sigma$ на $\mathscr{A}[\ell]$. Без труда проверяется, что таким образом мы определяем действие $\Gamma$ как группы автоморфизмов кольца $\mathscr{A}[\ell]$.

Пусть $\sigma \in \Gamma_{s}$ и $F(T) \in \mathscr{O}_{H}[[T]]$. Тогда $\varkappa(\sigma) \equiv 1\left(\bmod \ell^{s+1}\right)$, т.е. $\varkappa(\sigma)=$ $1+a \ell^{s+1}$ для некоторого $a \in \mathbb{Z}_{\ell}^{\times}$, и из (6.1) следует, что

$\sigma(T)-T=1-(1-T)(1-T)^{\ell^{s+1} a}-T=(1-T)\left[1-\left(1-T_{\langle s+1\rangle}\right)^{a}\right] \equiv 0 \quad\left(\bmod T_{\langle s+1\rangle}\right)$.

Отсюда следует, что $\sigma\left(T^{i}\right)-T^{i} \equiv 0\left(\bmod T_{\langle s+1\rangle}\right)$, что доказывает $(6.2)$. Сравнение (6.3) доказывается аналогично. Предложение доказано.

Как и в $\S 3$, мы введем формальную переменную $u$ и рассмотрим кольцо $\widetilde{\mathscr{B}}=\mathscr{O}_{H}[[u]]\{\{X\}\}$ и его подкольцо $\widetilde{\mathscr{A}}=\mathscr{O}_{H}[[u]]\{\{T\}\}$. Положим

$$
\sigma(T)=T-(1-T)\left[(1-u)^{a}-1\right] .
$$

Тогда $\sigma(T) \equiv T(\bmod u)$, и формула $(6.4)$ определяет действие $\sigma$ на кольца $\mathscr{O}_{H}[[u]][[T]]=\mathscr{O}_{H}[[u, T]]$ и $\mathscr{O}_{H}[[u]]\{\{T\}\}$. В частности, для многочлена $F(X, T)$ из предложения 1.2 определен многочлен $\mathbb{F}(X, T)=F(X, \sigma(T)) \in \mathscr{O}_{H}[[u, T]][X]$. То же рассуждение, что и в доказательстве предложения 6.1 , показывает, что в кольце $\mathscr{O}_{H}[[u, T]][X]$ выполняется сравнение

$$
\mathbb{F}(X, T) \equiv F(X, T) \quad(\bmod u) .
$$

ТЕОРема 6.1. В кольие $\widetilde{\mathscr{B}}$ существует однозначно определенный әлемент

$$
L(\lambda)=L(u, \lambda)=\sum_{j=-\infty}^{\infty} l_{j}(u) \lambda^{j}, \quad l_{j}(u) \in \mathscr{O}_{H}[[u]], \quad-\infty<j<\infty
$$

такой, что $L(\lambda) \equiv \lambda(\bmod u)$ и в $\widetilde{\mathscr{B}}$ выполняется равенство

$$
\mathbb{F}(L(\lambda), T(\lambda))=0 .
$$

Для любого $j<0$ коэффициент $l_{j}(u)$ удовлетворяет условию

$$
\nu\left(l_{j}(u)\right)>-\frac{j}{2 \delta},
$$

где функция $\nu$ имеет тот жее смысл, ито и в теореме 3.1 . 
Доказательство этой теоремы полностью аналогично доказательству теоремы 3.1.

Отметим, что из (6.5) следует

$$
0=F(\lambda, T) \equiv \mathbb{F}(\lambda, T) \quad(\bmod u),
$$

т.е. $\lambda$ является приближенным решением уравнения $\mathbb{F}(X, T)=0$ по модулю $u$.

В частном случае, когда $\varkappa(\sigma)=1+\ell^{s+1}\left(\varkappa(\sigma)=1+\ell^{s+2}\right.$ при $\left.\ell=2\right)$, мы имеем $L(u, \lambda)=\mu(u, \lambda)$, где $\mu(u, \lambda)$ - ряд из теоремы 3.1.

Пусть $p$ и $\gamma_{p}$ имеют тот же смысл, что и в теореме 2.1. Тогда согласно предложению $6.1 \gamma_{p}$ действует на $\mathscr{A}[\ell]$, причем для любого $f(T) \in \mathscr{O}_{H}[[T]]$

$$
\begin{array}{ll}
\gamma_{p}(f(T)) \equiv f(T)\left(\bmod T_{\langle p+1\rangle}\right) & \text { при } \quad \ell \neq 2, \\
\gamma_{p}(f(T)) \equiv f(T)\left(\bmod T_{\langle p+2\rangle}\right) & \text { при } \quad \ell=2 .
\end{array}
$$

Обозначим через $F_{1}(X, T)$ образ многочлена $F(X, T)$ относительно указанного действия $\gamma_{p}$ на коэффициенты. При этом

$$
\begin{aligned}
& F_{1}(X, T) \equiv F(X, T)\left(\bmod T_{\langle p+1\rangle}\right) \\
& F_{1}(X, T) \equiv F(X, T)\left(\bmod T_{\langle p+2\rangle}\right)
\end{aligned}
$$

До конца этого параграфа мы будем проводить все доказательства только для случая $\ell \neq 2$. Случай $\ell=2$ разбирается аналогично, и мы оставляем его читателю.

Пусть $\varkappa\left(\gamma_{p}\right)=1+a \ell^{p+1}$ для некоторого $a \in \mathbb{Z}_{\ell}$. Мы определим действие $\gamma_{p}$ на $T$ формулой (6.4), что определяет действие $\gamma_{p}$ на все кольцо $\widetilde{\mathscr{A}}$. Сопоставление $u \rightarrow T_{\langle p+1\rangle}$ индуцирует гомоморфизм колец $\psi: \widetilde{\mathscr{A}} \rightarrow \mathscr{A}[\ell]$, перестановочньй с действием $\gamma_{p}$ и такой, что

$$
\psi(\mathbb{F}(X, T))=F_{1}(X, T) .
$$

Положим

$$
\psi(L(\lambda))=L\left(T_{\langle p+1\rangle}, \lambda\right)=\sum_{j=-\infty}^{\infty} l_{j}\left(T_{\langle p+1\rangle}\right) \lambda^{j} .
$$

Из $(6.6)$ и того, что $T_{\langle p+1\rangle} \equiv T^{\ell^{p+1}}(\bmod \ell)$, легко выводится, что правая часть $(6.7)$ сходится в $\mathscr{B}[\ell]$. Это показывает, что элемент $\psi(L(\lambda))$ определен корректно.

Доказательство следующей теоремы аналогично доказательству теоремы 3.2.

ТЕОРема 6.2. Существует продолжение определенного выще действия $\gamma_{p}$ с $\widetilde{\mathscr{A}}$ на кольцо $\widetilde{\mathscr{B}}$. Это действие однозначно определяется условием $\gamma_{p}(\lambda) \equiv \lambda(\bmod u) . \quad$ явнном виде это действие задается формулой $\gamma_{p}(\lambda)=$ $L(u, \lambda)$. Существует продолжение определенного выше действия $\gamma_{p} c \mathscr{A}[\ell]$ на кольчо $\mathscr{B}[\ell]$ такое, что $\gamma_{p}(\lambda)=L\left(T_{\langle p+1\rangle}, \lambda\right)$. Этим условием действие $\gamma_{p}$ на $\mathscr{B}[\ell]$ определяется однозначно.

Существует единственное продолжение гомоморфизма $\psi: \widetilde{\mathscr{A}} \rightarrow \mathscr{A}[\ell]$ до гомоморфизма $\psi: \widetilde{\mathscr{B}} \rightarrow \mathscr{B}[\ell]$ такое, ито для любого $x \in \widetilde{\mathscr{B}}$

$$
\psi \gamma_{p}(x)=\gamma_{p} \psi(x) .
$$


ПРЕДЛОЖЕНИЕ 6.2. Пусть, как и в теореме 5.2, $s=n-p-1$. Тогда

$$
\begin{array}{lll}
\gamma_{p}\left(\lambda_{\langle s\rangle}(\lambda)\right)=L^{(s)}\left(T_{\langle n\rangle}, \lambda_{\langle s\rangle}(\lambda)\right) & \text { npu } & \ell \neq 2, \\
\gamma_{p}\left(\lambda_{\langle s\rangle}(\lambda)\right)=L^{(s)}\left(T_{\langle n+1\rangle}, \lambda_{\langle s\rangle}(\lambda)\right) & \text { npu } & \ell=2
\end{array}
$$

где $L^{(s)}(u, X)$ - ряд, полученный из $L(u, X)$ в результате действия $\varphi^{s}$ на коэффициенты.

ДокАЗАТЕЛЬСТво. Согласно (5.15)

$$
F^{(s)}\left(\lambda_{\langle s\rangle}(\lambda), T_{\langle s\rangle}(\lambda)\right)=0 .
$$

Обозначим через $\psi_{s}$ гомоморфизм $\mathscr{A}[\ell]$ в $\mathscr{A}[\ell]$ такой, что $\psi_{s}$ совпадает с $\varphi^{s}$ на $\mathscr{O}_{H}$ и $\psi_{s}(T)=T_{\langle s\rangle}$. Поскольку $\lambda$ является корнем неприводимого многочлена $F(X, T)$, который под действием $\psi_{s}$ переходит в многочлен $F^{(s)}\left(X, T_{\langle s\rangle}\right)$, гомоморфизм $\psi_{s}$ может быть продолжен до гомоморфизма $\psi_{s}: \mathscr{B}[\ell] \rightarrow \mathscr{B}[\ell]$ такого, что $\psi_{s}(\lambda)=\lambda_{\langle s\rangle}$.

Мы можем продолжить гомоморфизм $\psi_{s}: \mathscr{B}[\ell] \rightarrow \mathscr{B}[\ell]$ до гомоморфизма $\psi_{s}:$ $\widetilde{\mathscr{B}} \rightarrow \mathscr{B}[\ell]$ такого, что $\psi_{s}(u)=T_{\langle n\rangle}$ при $\ell \neq 2$ и $\psi_{s}(u)=T_{\langle n+1\rangle}$ при $\ell=2$. Тогда $\psi_{s}$ коммутирует с $\gamma_{p}$ и, следовательно, при $\ell \neq 2$

$$
\gamma_{p}\left(\lambda_{\langle s\rangle}(\lambda)\right)=\gamma_{p} \psi_{s}(\lambda)=\psi_{s} \gamma_{p}(\lambda)=\psi_{s}(L(u, \lambda))=L^{(s)}\left(T_{\langle n\rangle}(\lambda), \lambda_{\langle s\rangle}(\lambda)\right),
$$

а при $\ell=2$

$$
\gamma_{p}\left(\lambda_{\langle s\rangle}(\lambda)\right)=L^{(s)}\left(T_{\langle n+1\rangle}(\lambda), \lambda_{\langle s\rangle}(\lambda)\right) .
$$

Предложение доказано.

ПРЕДЛОЖЕНИЕ 6.3. Пусть $D\left(\lambda_{\langle s\rangle}\right)=D\left(\lambda_{\langle s\rangle}(\lambda)\right)-$ формальный ряд из теоремы 5.2. Тогда при $\ell \neq 2$ существует формальныи ряд $E\left(u, \lambda_{\langle s\rangle}\right) \in$ $\mathscr{O}_{H}[[u]]\left\{\left\{\lambda_{\langle s\rangle}\right\}\right\}$ mакой, что

$$
\gamma_{p}\left(D\left(\lambda_{\langle s\rangle}\right)\right)=E\left(T_{\langle n\rangle}\left(\lambda_{\langle s\rangle}\right), \lambda_{\langle s\rangle}\right) .
$$

Существует формальныи ряд $E_{1}\left(u, \lambda_{\langle s\rangle}\right) \in \mathscr{O}_{H}[[u]]\left\{\left\{\lambda_{\langle s\rangle}\right\}\right\}$ такой, что

$$
E\left(T_{\langle n\rangle}\left(\lambda_{\langle s\rangle}\right), \lambda_{\langle s\rangle}\right)=D\left(\lambda_{\langle s\rangle}\right)+T_{\langle n\rangle}\left(\lambda_{\langle s\rangle}\right) E_{1}\left(T_{\langle n\rangle}\left(\lambda_{\langle s\rangle}\right), \lambda_{\langle s\rangle}\right) .
$$

Существует формальный ряд $\mathscr{E}(X) \in \mathscr{O}_{H}\{\{X\}\}$ такой, что

$$
E_{1}\left(T_{\langle n\rangle}\left(\lambda_{\langle s\rangle}\right), \lambda_{\langle s\rangle}\right)=\mathscr{E}\left(\lambda_{\langle s\rangle}\right)
$$

При этом $\ell \mathscr{E}(X)=\ell \sum_{j=-\infty}^{\infty} e_{j} X^{j} \in \mathscr{O}_{H}\{\{X\}\}_{1}^{(0)}$, m.e. $\quad e_{j} \in \mathscr{O}_{H}$ для всех $j u$ $\nu_{\ell}\left(e_{j}\right)+1>-j /(2 \ell \delta)$ для всех $j<0$.

Аналогичный результат верен и для $\ell=2$, если заменить везде $T_{\langle n\rangle}$ на $T_{\langle n+1\rangle}$. 
ДоКАЗАТЕЛЬСТво. Предположим, что $\ell \neq 2$. Согласно теореме 5.3 выполняется условие $D(X) \in \mathscr{O}_{H}\{\{X\}\}_{1}^{(0)}$. Если $D(X)=\sum_{j=-\infty}^{\infty} d_{j} X^{j}$, то

$$
\gamma_{p}\left(D\left(\lambda_{\langle s\rangle}\right)\right)=\sum_{j=-\infty}^{\infty} d_{j}\left(L^{(s)}\left(T_{\langle n\rangle}\left(\lambda_{\langle s\rangle}\right), \lambda_{\langle s\rangle}\right)\right)^{j} .
$$

Если $j>0$, то, учитывая, что $L(u, \lambda) \equiv \lambda(\bmod u)$, получаем

$$
\begin{aligned}
d_{j} L^{(s)}\left(T_{\langle n\rangle}\left(\lambda_{\langle s\rangle}\right), \lambda_{\langle s\rangle}\right)^{j} & =d_{j}\left(\sum_{i=-\infty}^{\infty} l_{i}^{(s)}\left(T_{\langle n\rangle}\left(\lambda_{\langle s\rangle}\right)\right) \lambda_{\langle s\rangle}^{i}\right)^{j} \\
& =d_{j} \lambda_{\langle s\rangle}^{j}+d_{j} \sum_{i=-\infty}^{\infty} l_{i}^{\prime}\left(T_{\langle n\rangle}\left(\lambda_{\langle s\rangle}\right)\right) \lambda_{\langle s\rangle}^{i}
\end{aligned}
$$

где формальные степенные ряды $l_{i}^{\prime}\left(T_{\langle n\rangle}\right)$ делятся на $T_{\langle n\rangle}$ и в силу (6.6) удовлетворяют условию

$$
\nu\left(l_{i}^{\prime}(u)\right)>-\frac{i}{2 \delta} \quad \text { для всех } \quad i<0 .
$$

Если $j=0$, то

$$
d_{j} L^{(s)}\left(T_{\langle n\rangle}\left(\lambda_{\langle s\rangle}\right), \lambda_{\langle s\rangle}\right)^{j}=d_{j} .
$$

Наконец, если $j<0$, то, учитьвая, что $l_{1}\left(T_{\langle n\rangle}\right) \equiv 1\left(\bmod T_{\langle n\rangle}\right)$, а следовательно, этот ряд обратим в $\mathscr{O}_{H}\left[\left[T_{\langle n\rangle}\right]\right]$, мы получаем

$$
L^{(s)}(Y, X)=l_{1}(Y) X\left(1+\sum_{\substack{\alpha=-\infty \\ \alpha \neq 0}}^{\infty} l_{1}(Y)^{-1} l_{\alpha+1}(Y) X^{\alpha}\right) .
$$

Из (6.6) следует, что при $\alpha \leqslant-2$

$$
\nu\left(l_{1}(Y)^{-1} l_{\alpha+1}(Y)\right)>-\frac{\alpha+1}{2 \delta} \geqslant-\frac{\alpha}{2 \delta \ell} .
$$

Если $\alpha=-1$, то

$$
\nu\left(l_{1}(Y)^{-1} l_{0}(Y)\right) \geqslant 1>\frac{1}{2 \delta \ell} .
$$

Другими словами, если мы положим

$$
N(Y, X)=\sum_{\substack{\alpha=-\infty \\ \alpha \neq 0}}^{\infty} l_{1}(Y)^{-1} l_{\alpha+1}(Y) X^{\alpha}=\sum_{i=-\infty}^{\infty} n_{i}(Y) X^{i},
$$

то для любого $i<0$ выполняется неравенство

$$
\nu\left(n_{i}(Y)\right)>-\frac{i}{2 \delta \ell}
$$


Тогда из (6.9) получаем

$$
L^{(s)}(Y, X)^{-1}=l_{1}(Y)^{-1} X^{-1}\left(1+\sum_{\nu=1}^{\infty}(-1)^{\nu} N(Y, X)^{\nu}\right),
$$

откуда следует, что при $j<0$

$$
\begin{aligned}
d_{j} L^{(s)}\left(T_{\langle n\rangle}, \lambda_{\langle s\rangle}\right)^{j}= & d_{j} \lambda_{\langle s\rangle}^{j}+d_{j}\left[l_{1}\left(T_{\langle n\rangle}\right)^{j}-1\right] \lambda_{\langle s\rangle}^{j} \\
& +d_{j} l_{1}\left(T_{\langle n\rangle}\right)^{j} \lambda_{\langle s\rangle}^{j} \sum_{\nu=1}^{\infty}(-1)^{\nu} N\left(T_{\langle n\rangle}, \lambda_{\langle s\rangle}\right)^{\nu} .
\end{aligned}
$$

Из этого, как и в доказательстве теоремы 5.2 , следует, что ряд $d_{j} L^{\langle s\rangle}\left(T_{\langle n\rangle}, \lambda_{\langle s\rangle}\right)^{j}$ удовлетворяет условию вида (6.10). Таким образом, мы получаем

$$
E\left(T_{\langle n\rangle}, \lambda_{\langle s\rangle}\right)=D\left(\lambda_{\langle s\rangle}\right)+T_{\langle n\rangle} E_{1}\left(T_{\langle n\rangle}, \lambda_{\langle s\rangle}\right),
$$

где ряд $T_{\langle n\rangle} E_{1}\left(T_{\langle n\rangle}, \lambda_{\langle s\rangle}\right)$ удовлетворяет условию вида (6.10). Очевидно, что тогда и ряд $\ell E_{1}\left(T_{\langle n\rangle}, \lambda_{\langle s\rangle}\right)$ удовлетворяет условию вида (6.10). Учитывая, что

$$
T_{\langle n\rangle}=1-\left(1-T_{\langle s\rangle}\right)^{\ell^{p+1}},
$$

мы можем рассматривать $E_{1}\left(T_{\langle n\rangle}, \lambda_{\langle s\rangle}\right)$ как ряд от $T_{\langle s\rangle}$ и $\lambda_{\langle s\rangle}$. Далее, если $T=T(\lambda)$, то $T_{\langle s\rangle}=T^{(s)}\left(\lambda_{\langle s\rangle}\right)$, поэтому сушествует ряд $\mathscr{E}_{1}(X)$ такой, что $\mathscr{E}_{1}\left(\lambda_{\langle s\rangle}\right)=E_{1}\left(T_{\langle n\rangle}\left(\lambda_{\langle s\rangle}\right), \lambda_{\langle s\rangle}\right)$.

То, что ряд $\ell E_{1}\left(T_{\langle n\rangle}, \lambda_{\langle s\rangle}\right)$ удовлетворяет условию вида (6.10), означает, что этот ряд можно представить как сумму одночленов вида $a \ell^{k} T_{\langle n\rangle}^{r} \lambda_{\langle s\rangle}^{j}$, где $a \in \mathbb{Z}_{\ell}^{\times}$, $k \geqslant 1$ и

$$
k+r>-\frac{j}{2 \delta \ell} \text { для любого } j<0 .
$$

Согласно (6.11)

$$
T_{\langle n\rangle}=T_{\langle s\rangle}^{\ell^{p+1}}+\ell P\left(T_{\langle s\rangle}\right)=\lambda_{\langle s\rangle}^{e \ell^{p+1}}+\ell P_{1}\left(\lambda_{\langle s\rangle}\right)
$$

для некоторых $P(X) \in \mathscr{O}_{H}[X]$ и $P_{1}(X) \in \mathscr{O}_{H}[[X]]$.

Из неравенств $\nu_{\ell}\left(\lambda_{q}^{\delta}\right)<1 / 2$ и $p \geqslant q+2$ следует, что

$$
2 \delta \ell<e \ell^{p+1}
$$

Тогда из (6.12) и (6.13) следует, что

$$
a \ell^{k} T_{\langle n\rangle}^{r} \lambda_{\langle s\rangle}^{j}=a \ell^{k+r} P_{2}\left(\lambda_{\langle s\rangle}\right) \lambda_{\langle s\rangle}^{j}+a \ell^{k} P_{3}\left(\lambda_{\langle s\rangle}\right) \lambda_{\langle s\rangle}^{2 r \delta \ell+j}
$$

для некоторых $P_{2}(X), P_{3}(X) \in \mathscr{O}_{H}[[X]]$. Из (6.12) вытекает, что оба слагаемых в правой части (6.14) удовлетворяют неравенству вида (6.10). Предложение доказано. 
ТеОРема 6.3. Пусть $S_{j}$ имеет тот же смысл, ито и в теореме 5.4. Тогда для любого $j \geqslant n$

$$
\begin{aligned}
& \gamma_{p} S_{j}=D^{(-j)}\left(L^{(s-j)}\left(\lambda_{j-s}\right)\right), \\
& \left(\gamma_{p}-1\right) S_{j}=\pi_{j-n \mathscr{E}} \mathscr{E}^{(-j)}\left(\lambda_{j-s}\right) \quad \text { npu } \quad \ell \neq 2, \\
& \left(\gamma_{p}-1\right) S_{j}=\pi_{j-n-1} \mathscr{E}^{(-j)}\left(\lambda_{j-s}\right) \quad \text { npu } \quad \ell=2 .
\end{aligned}
$$

ДокАЗАТЕЛЬСТво. Действуя автоморфизмом $\varphi^{-j}$ на коэффициенты тождества (6.8) и применяя к нему автоморфизм $\gamma_{p}$, мы получаем

$$
F^{(s-j)}\left(L^{(s-j)}\left(\lambda_{\langle s\rangle}^{(-j)}(\lambda)\right), \gamma_{p}\left(T_{\langle s\rangle}^{(-j)}(\lambda)\right)\right)=0 .
$$

Подставляя $\lambda=\lambda_{j}$ и учитывая, что при $j \geqslant n$ все ряды, входящие в (6.15), сходятся, учитывая также, что в доказательстве теоремы 5.3 было показано, что $\lambda_{\langle s\rangle}^{(-j)}\left(\lambda_{j}\right)=\lambda_{j-s}$ и что $T_{\langle s\rangle}^{(-j)}\left(\lambda_{j}\right)=\pi_{j-s}$, мы получаем из (6.15) равенство

$$
F^{(s-j)}\left(L^{(s-j)}\left(\lambda_{j-s}\right), \gamma_{p}\left(\pi_{j-s}\right)\right)=0 .
$$

С другой стороны, мы имеем равенство

$$
F^{(s-j)}\left(\gamma_{p}\left(\lambda_{j-s}\right), \gamma_{p}\left(\pi_{j-s}\right)\right)=0
$$

Тогда из сравнения $L(u, \lambda) \equiv \lambda(\bmod u)$ следует, что

$$
\gamma_{p}\left(\lambda_{j-s}\right)=L^{(s-j)}\left(\lambda_{j-s}\right) .
$$

Согласно теореме $5.3 S_{j}=D^{(-j)}\left(\lambda_{j-s}\right)$, поэтому

$$
\gamma_{p} S_{j}=D(-j)\left(\gamma_{p}\left(\lambda_{j-s}\right)\right)=D^{(-j)}\left(L^{(s-j)}\left(\lambda_{j-s}\right)\right),
$$

что доказывает первое равенство теоремы. Вторые два равенства следуют из того, что при $T=\pi_{j}$ мы имеем $T_{\langle n\rangle}(T)=\pi_{j-n}$ и $T_{\langle n+1\rangle}(T)=\pi_{j-n-1}$. Теорема доказана.

\section{§ 7. Доказательство основной теоремы}

Пусть элементы $\eta_{n, 1}=\eta_{n}\left(\alpha_{1}, a_{1}\right), \ldots, \eta_{n, t}=\eta_{n}\left(\alpha_{t}, a_{t}\right)$ вида (2.1) порожда-

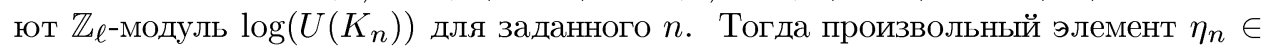
$\log \left(U\left(K_{n}\right)\right)$ может быть записан в виде

$$
\eta_{n}=\sum_{i=1}^{t} c_{i} \eta_{n, i}
$$

с некоторыми $c_{i} \in \mathbb{Z}_{\ell}$. 
Согласно теореме 6.3 для каждого $i=1, \ldots, t$ существует ряд Лорана $\mathscr{E}_{i}(X)$ такой, что $\ell_{\mathscr{E}}(X) \in \mathscr{O}_{H}\{\{X\}\}_{1}^{(0)}$ и

$$
\left(\gamma_{p}-1\right) \mathscr{F}_{j}^{(s)}\left(\eta_{j, i}\right)=\pi_{j-n} \mathscr{E}_{i}^{(-j)}\left(\lambda_{j-s}\right), \quad s=n-p-1,
$$

где $\eta_{j, i}=\eta_{j}\left(\varphi^{n-j}\left(\alpha_{i}\right), a_{i}\right)$. Тогда, полагая для любого $j \geqslant n$

$$
\eta_{j}=\sum_{i=1}^{t} c_{i} \eta_{j, i}, \quad \mathscr{E}(X)=\sum_{i=1}^{t} c_{i} \mathscr{E}_{i}(X)
$$

мы получаем для любого $j \geqslant n$

$$
\left(\gamma_{p}-1\right) \mathscr{F}_{j}^{(s)}\left(\eta_{j}\right)=\pi_{j-n} \mathscr{E}^{(-j)}\left(\lambda_{j-s}\right),
$$

причем $\ell \mathscr{E}(X) \in \mathscr{O}_{H}\{\{X\}\}_{1}^{(0)}$.

Если $e_{\nu}-$ коэффициент ряда $\mathscr{E}(X)$ при $X^{\nu}$ и $e_{i, \nu}-$ коэффициент при $X^{\nu}$ ряда $\mathscr{E}_{i}(X)$ для $i=1, \ldots, t$, то

$$
e_{\nu}=\sum_{i=1}^{t} c_{i} e_{i, \nu}
$$

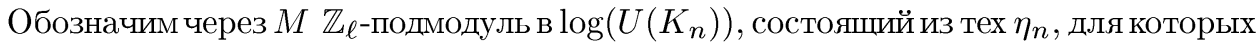
ряд $\mathscr{E}(X)$ имеет нулевые коэффициенты $e_{\nu}$ для всех $\nu$ таких, что $|\nu| \leqslant 4(n-p+2) \ell \delta$. Тогда в $\mathscr{E}(X)$ должны быть равны нулю $8(n-p+2) \ell \delta+1$ коэффициент $e_{\nu}$, т.е. $M$ задается в $\log \left(U\left(K_{n}\right)\right) \quad 8(n-p+2) \ell \delta+1$ линейньт уравнением относительно $c_{1}, \ldots, c_{t}$ :

$$
e_{\nu}=\sum_{i=1}^{t} c_{i} e_{i, \nu}=0, \quad|\nu| \leqslant 2(n-p+2) .
$$

Поскольку коэффициенты $e_{\nu}$ и $e_{i, \nu}$ принадлежат $\mathscr{O}_{H}$, любое из уравнений (7.3) эквивалентно $f=\left[H: \mathbb{Q}_{\ell}\right]$ линейным уравнениям с коэффишиентами в $\mathbb{Z}_{\ell}$.

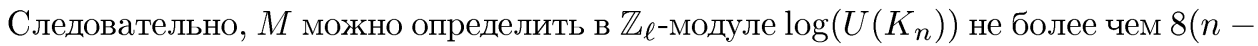
$p+2) \ell \delta f+f$ линейными уравнениями.

ТЕОРема 7.1. Пусть $\eta_{n}-$ произвольный әлемент из $M$ и $\eta_{n, i}-$ любой из әлементов $\eta_{n, 1}, \ldots, \eta_{n, t}$. Пусть, как и в $(2.2)$,

$$
A^{\prime}=A\left(\eta_{n}, \eta_{n, i}\right)=\operatorname{Sp}_{K_{n} / \mathbb{Q}_{\ell}}\left(\Theta_{n}(p)\left(\eta_{n}\right) \eta_{n, i}\right) .
$$

Тогда $A^{\prime} \in \mathbb{Z}_{\ell}$. Другими словами,

$$
\left(\gamma_{p}-1\right) M \subseteq\left(U\left(K_{n}\right)\right)^{\perp} .
$$

ДоКАЗАТЕЛЬСТВО. Пусть $\eta_{n, i}-$ один из элементов $\eta_{n, 1}, \ldots, \eta_{n, t}$ и $D(X)$ - ряд, соответствующий элементу $\eta_{n, i}$ согласно теореме 5.3 , т.е. такой, что для любого $j \geqslant n$

$$
\mathscr{F}_{j}^{(s)}\left(\eta_{j, i}\right)=D^{(-j)}\left(\lambda_{j-s}\right) .
$$


Тогда согласно теореме 2.1 и формулам (7.1), (7.4) мы имеем

$$
A^{\prime} \equiv \ell^{-m-n+p-1} \sum_{j=n}^{\infty} \operatorname{Sp}_{K_{j} / \mathbb{Q}_{\ell}}\left(\pi_{j-n} \mathscr{E}^{(-j)}\left(\lambda_{j-s}\right) D^{(-j)}\left(\lambda_{j-s}\right)\right) \quad\left(\bmod \mathbb{Z}_{\ell}\right)
$$

где $m$ связано с $n$ соотношением (1.1), и при $\ell=2 \pi_{j-n}$ нужно заменить на $\pi_{j-n-1}$.

Если $\mathscr{E}(X)=\sum_{\nu=-\infty}^{\infty} e_{\nu} X^{\nu}$, то положим

$$
\mathscr{E}(X)=\mathscr{E}_{1}(X)+\mathscr{E}_{2}(X)
$$

где

$$
\mathscr{E}_{1}(X)=\sum_{\nu=-\infty}^{-4(n-p+2)-1} e_{\nu} X^{\nu}, \quad \mathscr{E}_{2}(X)=\sum_{\nu=4(n-p+2)+1}^{\infty} e_{\nu} X^{\nu}
$$

Если $D(X)=\sum_{\nu=-\infty}^{\infty} d_{\nu} X^{\nu}$, то мы положим

$$
D(X)=D_{1}(X)+D_{2}(X),
$$

где

$$
D_{1}(X)=\sum_{\nu=-\infty}^{-4(n-p+2)-2} d_{\nu} X^{\nu}, \quad D_{2}(X)=\sum_{\nu=-4(n-p+2)-1}^{\infty} d_{\nu} X^{\nu} .
$$

Тогда

$$
A^{\prime}=\sum_{1 \leqslant \alpha, \beta \leqslant 2} A_{\alpha, \beta}^{\prime}
$$

где

$$
\begin{gathered}
A_{\alpha, \beta}^{\prime} \equiv \ell^{-m-n+p-1} \sum_{j=n}^{\infty} \operatorname{Sp}_{K_{j} / \mathbb{Q}_{\ell}}\left(\pi_{j-n} C_{\alpha, \beta}^{(-j)}\left(\lambda_{j-s}\right)\right)\left(\bmod \mathbb{Z}_{\ell}\right) \\
C_{\alpha, \beta}(X)=\mathscr{E}_{\alpha}(X) D_{\beta}(X), \quad 1 \leqslant \alpha, \beta \leqslant 2 .
\end{gathered}
$$

Поскольку $\nu_{\ell}\left(\lambda_{q}^{2 \delta}\right)<1$ и $p>q+2$, мы имеем $\nu_{\ell}\left(\lambda_{p+1}^{2 \ell \delta}\right)<1 / \ell^{2}$. Если $j \geqslant n$, то $j-s \geqslant p+1$, поэтому

$$
\nu_{\ell}\left(\lambda_{j-s}^{2 \ell \delta}\right)<\frac{1}{\ell^{2}} \text { при любом } j \geqslant n .
$$

Рассмотрим произвольный одночлен $e_{\nu} \lambda_{j-s}^{\nu}$, входящий в $\mathscr{E}_{1}(X)$. Пусть $0>\nu=\nu_{0}+\nu_{1} 2 \ell \delta$, где $-2 \ell \delta<\nu_{0} \leqslant 0$.

Если $\nu_{0}=0$, то из (7.8) следует, что $\nu_{\ell}\left(\lambda_{j-s}^{\nu}\right)>\nu_{1} / \ell^{2}$ и $\nu_{\ell}\left(e_{\nu}\right)+1>$ $-\nu /(2 \ell \delta)=-\nu_{1}$, т.е. $\nu_{\ell}\left(e_{\nu}\right) \geqslant-\nu_{1}$. Тогда $\nu_{\ell}\left(e_{\nu} \lambda_{j-s}^{\nu}\right)>-(1 / 2) \nu_{1}=-\nu /(4 \ell \delta)$. В частности,

$$
\nu_{\ell}\left(e_{\nu} \lambda_{j-s}^{\nu}\right) \geqslant n-p+2 .
$$

Если $\nu_{0} \neq 0$, то $\nu_{\ell}\left(\lambda_{j-s}^{\nu}\right)>\left(\nu_{1}-1\right) / \ell^{2}$ и $\nu_{\ell}\left(e_{\nu}\right)+1>-\nu /(2 \ell \delta)>-\nu_{1}$, т.e. $\nu_{\ell}\left(e_{\nu}\right)>-\nu_{1}$. Тогда $\nu_{\ell}\left(e_{\nu} \lambda_{j-s}^{\nu}\right)>-\nu_{1}\left(\ell^{2}-1\right) / \ell^{2}-1 / \ell^{2}$. Учитывая, что $\nu_{1} \leqslant-2(n-p+2)$, мы получаем, что и в этом случае справедлива оценка (7.9). Отметим, что из неравенства $p \geqslant q+3$ можно вывести, что оценка (7.9) справедлива и для $\ell=2$. 
Итак, мы доказали, что

$$
\mathscr{E}_{1}^{(-j)}\left(\lambda_{j-s}\right) \equiv 0 \quad\left(\bmod \ell^{n-p+2}\right)
$$

Аналогичные оценки, проведенные для $D_{1}(X)$, показывают, что для любого $j \geqslant n$

$$
D_{1}^{(-j)}\left(\lambda_{j-s}\right) \equiv 0 \quad\left(\bmod \ell^{n-p+2}\right)
$$

Поскольку $\mathscr{E}_{2}(X) \in \mathscr{O}_{H}[[X]]$ и $D_{2}(X) \in \mathscr{O}_{H}\{\{X\}\}_{1}^{(0)}$, мы имеем

$$
\mathscr{E}_{2}^{(-j)}\left(\lambda_{j-s}\right) \equiv D_{2}^{(-j)}\left(\lambda_{j-s}\right) \equiv 0 \quad\left(\bmod \mathbb{Z}_{\ell}\right)
$$

Следовательно,

$$
C_{1,1}^{(-j)}\left(\lambda_{j-s}\right) \equiv C_{1,2}^{(-j)}\left(\lambda_{j-s}\right) \equiv C_{2,1}^{(-j)}\left(\lambda_{j-s}\right) \equiv 0 \quad\left(\bmod \ell^{n-p+2}\right) .
$$

Учитывая, что $\operatorname{Sp}_{K_{j} / \mathbb{Q}_{\ell}}\left(\ell^{1-m} \mathscr{O}_{K_{j}}\right) \subseteq \mathbb{Z}_{\ell}$ для всех $j \geqslant n$, получаем

$$
A_{1,1}^{\prime} \equiv A_{1,2}^{\prime} \equiv A_{2,1}^{\prime} \equiv 0 \quad\left(\bmod \mathbb{Z}_{\ell}\right)
$$

Следовательно,

$$
A^{\prime} \equiv A_{2,2}^{\prime} \equiv \ell^{-m-n+p-1} \sum_{j=n}^{\infty} \operatorname{Sp}_{K_{j} / \mathbb{Q}_{\ell}}\left(\pi_{j-n} C_{2,2}^{(-j)}\left(\lambda_{j-s}\right)\right) \quad\left(\bmod \mathbb{Z}_{\ell}\right)
$$

Непосредственно из определения $\mathscr{E}_{2}(X)$ и $D_{2}(X)$ следует, что $C_{2,2}(X)$ является формальным степенным рядом, т.е. $C_{2,2}(X)$ не содержит отрицательных степеней $X$.

ПРЕДЛОЖЕНИЕ 7.1. При $\ell \neq 2$ существует формальный степенной ряд $P(X) \in \mathscr{O}_{H}[[X]]$ такой, что для любого $j \geqslant n$

$$
\pi_{j-n} \operatorname{Sp}_{K_{j} / k_{j}}\left(C_{2,2}^{(-j)}\left(\lambda_{j-s}\right)\right)=1-\left(1-\pi_{j-s}\right)^{\ell^{n-s}} P^{(-j)}\left(\pi_{j-s}\right) .
$$

Этот результат остается верен и при $\ell=2$, если заменить $\pi_{j-n}$ на $\pi_{j-n-1}$, а показатель $\ell^{n-s}$ на $\ell^{n-s+1}$.

ДоКАЗАТЕЛЬСТВо. Очевидно, что $\pi_{j-n}=1-\left(1-\pi_{j-s}\right)^{\ell^{n-s}}$ для любого $j \geqslant n$. Согласно предложению 1.2 для любого $j-s \geqslant q$ выполняется равенство $F^{(s-j)}\left(\lambda_{j-s}, \pi_{j-s}\right)=0$. Пусть $\lambda_{j-s}=\lambda_{j-s}^{(1)}, \lambda_{j-s}^{(2)}, \ldots, \lambda_{j-s}^{(e)}-$ все корни многочлена $F^{(s-j)}\left(X, \pi_{j-s}\right)$. Тогда для любого $\nu$ величина

$$
\operatorname{Sp}_{K_{j} / k_{j}}\left(\lambda_{j-s}^{\nu}\right)=\sum_{i=1}^{e}\left(\lambda_{j-s}^{(i)}\right)^{\nu}
$$


является симметрической функцией от $\lambda_{j-s}^{(i)}, i=1, \ldots, e$, и, следовательно, выражается через элементарные симметрические функции, т.е. существует многочлен $Q_{\nu}\left(X_{1}, \ldots, X_{e}\right) \in \mathbb{Z}\left[X_{1}, \ldots, X_{e}\right]$ такой, что для любого $j \geqslant n$

$$
\operatorname{Sp}_{K_{j} / k_{j}}\left(\lambda_{j-s}^{\nu}\right)=Q_{\nu}\left(f_{e-1}^{(s-j)}\left(\pi_{j-s}\right), \ldots, f_{0}^{(s-j)}\left(\pi_{j-s}\right)\right)
$$

где $f_{e-1}(T), \ldots, f_{0}(T) \in \mathscr{O}_{H}[[T]]$ - коэффициенты многочлена $F(X, T)$. Таким образом, полагая $\widetilde{Q}_{\nu}(T)=Q_{\nu}\left(f_{e-1}^{(s)}(T), \ldots, f_{0}^{(s)}(T)\right)$, получаем

$$
\operatorname{Sp}_{K_{j} / k_{j}}\left(\lambda_{j-s}^{\nu}\right)=\widetilde{Q}_{\nu}^{(-j)}\left(\pi_{j-s}\right) .
$$

Если $C_{2,2}(X)=\sum_{\nu=0}^{\infty} c_{\nu} X^{\nu}$, где $c_{\nu} \in \mathscr{O}_{H}$, то

$$
\pi_{j-n} \operatorname{Sp}_{K_{j} / k_{j}}\left(C_{2,2}^{(-j)}\left(\lambda_{j-s}\right)\right)=1-\left(1-\pi_{j-s}\right)^{\ell^{n-s}} P^{(-j)}\left(\pi_{j-s}\right),
$$

где $P(X)=\sum_{\nu=0}^{\infty} c_{\nu} \widetilde{Q}_{\nu}(X)$. Предложение доказано.

Таким образом, полагая

$$
P_{1}(X)=\left[1-(1-X)^{\ell^{n-s}}\right] P(X),
$$

мы получаем

$$
A^{\prime} \equiv A_{2,2}^{\prime} \equiv \ell^{-m-n+p-1} \sum_{j=n}^{\infty} \operatorname{Sp}_{k_{j} / \mathbb{Q}_{\ell}}\left(P_{1}^{(-j)}\left(\pi_{j-s}\right)\right) .
$$

При $\ell=2$ показатель $\ell^{n-s}$ в (7.12) следует заменить на $\ell^{n-s+1}$.

Для дальнейшего нам потребуется следующее утверждение [7, лемма 6].

ЛЕмма 7.1. Пусть $f(T) \in \mathscr{O}_{H}[[T]] u \pi_{-1}=0$. Тогда

$$
s_{\infty}(f):=\delta+\sum_{i=-1}^{\infty} \operatorname{Sp}_{k_{i} / \mathbb{Q}_{\ell}}\left(f^{(-i)}\left(\pi_{i}\right)\right)=0,
$$

əде $k_{-1}=H u \delta=0$ npu $\ell \neq 2, \delta=\operatorname{Sp}_{H / \mathbb{Q}_{\ell}}\left(f^{(1)}(2)\right) n p u \ell=2$.

ЛЕмма 7.2. Пусть $P_{1}(X)$ - степенной ряд из (7.12). Тогда для всех $-1 \leqslant i<n-s \quad P_{1}\left(\pi_{i}\right)=0$. Если $\ell=2$, то, кроме того, $P_{1}(2)=0$.

ДокаЗАТЕЛЬСтво. Пусть $\ell \neq 2$. Тогда $n-s=p+1$, поэтому, учитывая, что $1-\pi_{i}=\zeta_{i}$ и $\zeta_{i}^{\ell^{p+1}}=1$, мы получаем, что множитель $1-\left(1-\pi_{i}\right)^{\ell^{p+1}}$ в $(7.12)$ равен нулю для любого $-1 \leqslant i<n-s$. Тогда и $P_{1}\left(\pi_{i}\right)=0$ для этих значений $i$.

Если $\ell=2$, то $\zeta_{i}$ имеет порядок $\ell^{i+2}$, откуда снова следует, что $1-\left(1-\pi_{i}\right)^{\ell^{p+2}}=0$ для $-1 \leqslant i<n-s$. Если $X=2$, то снова $1-(1-2)^{2^{p+2}}=0$. Лемма доказана.

Теперь мы легко можем закончить доказательство теоремы 7.1. В силу леммы 7.1 мы имеем $s_{\infty}\left(P_{1}\right)=0$, а лемма 7.2 показывает, что $P_{1}\left(\pi_{i}\right)=0$ для $-1 \leqslant i<n-s$. Причем при $\ell=2$ мы имеем $P_{1}(2)=0$. Тогда из (7.13) следует, что

$$
\begin{aligned}
A^{\prime} & \equiv \ell^{-m-n+p-1} \sum_{j=n}^{\infty} \operatorname{Sp}_{k_{j} / \mathbb{Q}_{\ell}}\left(P_{1}^{(-j)}\left(\pi_{j-s}\right)\right) \\
& \equiv \ell^{-m-n+p-1} s_{\infty}\left(P_{1}\right) \equiv 0\left(\bmod \mathbb{Z}_{\ell}\right)
\end{aligned}
$$

Это доказывает теорему 7.1, а вместе с ней и основную теорему. 


\section{Список литературы}

1. Iwasawa K. On some modules in the theory of cyclotomic fields // J. Math. Soc. Japan. 1964. V. 20. P. 42-82.

2. Кузьмин Л. В. Некоторые замечания о $l$-адической теореме Дирихле и $l$-адическом регуляторе // Изв. АН СССР. Сер. матем. 1981. Т. 45. №6. С. 1203-1240.

3. Кузьмин Л. В. Некоторые замечения о $l$-адическом регуляторе. II // Изв. АН СССР. Сер. матем. 1989. Т. 53. №4. С. 782-813.

4. Кузьмин Л. В. Аналог формулы Римана-Гурвица для одного типа $l$-расширений полей алгебраических чисел // Изв. АН СССР. Сер. матем. 1990. Т. 54. № 2. С. 316-338.

5. Кузьмин Л. В. Новые явные формулы для символа норменного вычета и их приложения // Изв. АН СССР. Сер. матем. 1990. Т. 54. №6. С. 1196-1228.

6. Кузьмин Л. В. Некоторые явные вычисления в локальных и глобальных круговых полях // Тр. Матем. ин-та им. В. А. Стеклова РАН. 1995. Т. 208. С. 202-223.

7. Кузьмин Л. В. Новое доказательство одной теоремы двойственности о $\ell$-адических логарифмах локальных единиц // Итоги науки и техники. Совр. мат. и ее приложения. 1997. T. 45. C. $72-81$.

8. Tate J. p-divisible groups // Proc. of Conference on local Fields. Berlin: Springer, 1967. 158-183 // Русский перев.: Математика. 1969. Т. 13. № 2. С. 3-25.

9. Wintenberger J.-P. Le corps des normes de certaines extensions infinies de corps locaux; application // Ann. Sci. Ec. Norm. Super. IV. Ser. 16. 1983. P. 59-89.

Поступило в редакцию 